\title{
Exoplanet Biosignatures: Understanding Oxygen as a Biosignature in the Context of Its Environment
}

\author{
Victoria S. Meadows, ${ }^{1,2}$ Christopher T. Reinhard, ${ }^{3,4}$ Giada N. Arney, ${ }^{2,5}$ Mary N. Parenteau, ${ }^{2,6}$ \\ Edward W. Schwieterman,,4,7-9 Shawn D. Domagal-Goldman, ${ }^{2,10}$ Andrew P. Lincowski, ${ }^{1,2}$ \\ Karl R. Stapelfeldt, ${ }^{11}$ Heike Rauer, ${ }^{12}$ Shiladitya DasSarma, ${ }^{13,14}$ Siddharth Hegde, ${ }^{15,16}$ Norio Narita, ${ }^{17-19}$ \\ Russell Deitrick, ${ }^{1,2}$ Jacob Lustig-Yaeger, ${ }^{1,2}$ Timothy W. Lyons, ${ }^{4,7}$ Nicholas Siegler, ${ }^{11}$ and J. Lee Grenfell ${ }^{12}$
}

\begin{abstract}
We describe how environmental context can help determine whether oxygen $\left(\mathrm{O}_{2}\right)$ detected in extrasolar planetary observations is more likely to have a biological source. Here we provide an in-depth, interdisciplinary example of $\mathrm{O}_{2}$ biosignature identification and observation, which serves as the prototype for the development of a general framework for biosignature assessment. Photosynthetically generated $\mathrm{O}_{2}$ is a potentially strong biosignature, and at high abundance, it was originally thought to be an unambiguous indicator for life. However, as a biosignature, $\mathrm{O}_{2}$ faces two major challenges: (1) it was only present at high abundance for a relatively short period of Earth's history and (2) we now know of several potential planetary mechanisms that can generate abundant $\mathrm{O}_{2}$ without life being present. Consequently, our ability to interpret both the presence and absence of $\mathrm{O}_{2}$ in an exoplanetary spectrum relies on understanding the environmental context. Here we examine the coevolution of life with the early Earth's environment to identify how the interplay of sources and sinks may have suppressed $\mathrm{O}_{2}$ release into the atmosphere for several billion years, producing a false negative for biologically generated $\mathrm{O}_{2}$. These studies suggest that planetary characteristics that may enhance false negatives should be considered when selecting targets for biosignature searches. We review the most recent knowledge of false positives for $\mathrm{O}_{2}$, planetary processes that may generate abundant atmospheric $\mathrm{O}_{2}$ without a biosphere. We provide examples of how future photometric, spectroscopic, and time-dependent observations of $\mathrm{O}_{2}$ and other aspects of the planetary environment can be used to rule out false positives and thereby increase our confidence that any observed $\mathrm{O}_{2}$ is indeed a biosignature. These insights will guide and inform the development of future exoplanet characterization missions. Key Words: BiosignaturesOxygenic photosynthesis-Exoplanets-Planetary atmospheres. Astrobiology 18, 630-662.
\end{abstract}

\footnotetext{
${ }^{1}$ Department of Astronomy, University of Washington, Seattle, Washington.

${ }^{2}$ NASA Astrobiology Institute, Virtual Planetary Laboratory Team, Seattle, Washington.

${ }^{3}$ School of Earth and Atmospheric Sciences, Georgia Institute of Technology, Atlanta, Georgia.

${ }^{4}$ NASA Astrobiology Institute, Alternative Earths Team, Riverside, California.

${ }^{5}$ Planetary Systems Laboratory, NASA Goddard Space Flight Center, Greenbelt, Maryland.

${ }^{6}$ NASA Ames Research Center, Exobiology Branch, Mountain View, California.

${ }^{7}$ Department of Earth Sciences, University of California, Riverside, California.

${ }^{8}$ NASA Postdoctoral Program, Universities Space Research Association, Columbia, Maryland.

${ }^{9}$ Blue Marble Space Institute of Science, Seattle, Washington.

${ }^{10}$ Planetary Environments Laboratory, NASA Goddard Space Flight Center, Greenbelt, Maryland.

${ }^{11}$ NASA Exoplanet Exploration Program, Jet Propulsion Laboratory/California Institute of Technology, Pasadena, California.

${ }^{12}$ German Aerospace Center, Institute of Planetary Research, Extrasolar Planets and Atmospheres, Berlin, Germany.

${ }^{13}$ Department of Microbiology and Immunology, School of Medicine, University of Maryland, Baltimore, Maryland.

${ }^{14}$ Institute of Marine and Environmental Technology, University System of Baltimore, Maryland.

${ }^{15}$ Carl Sagan Institute, Cornell University, Ithaca, New York.

${ }^{16}$ Cornell Center for Astrophysics and Planetary Science, Cornell University, Ithaca, New York.

${ }^{17}$ Department of Astronomy, The University of Tokyo, Tokyo, Japan.

${ }^{18}$ Astrobiology Center, NINS, Tokyo, Japan.

${ }^{19}$ National Astronomical Observatory of Japan, NINS, Tokyo, Japan.
}

(C) Victoria S. Meadows et al., 2018; Published by Mary Ann Liebert, Inc. This Open Access article is distributed under the terms of the Creative Commons Attribution Noncommercial License (http://creativecommons.org/licenses/by-nc/4.0/) which permits any noncommercial use, distribution, and reproduction in any medium, provided the original author(s) and the source are credited. 


\section{Introduction}

$\mathbf{T}$ HE SEARCH FOR life across interstellar distances is a scientific challenge that pushes the boundaries of observational astronomy and requires careful consideration of the signs of life that we will best be able to detect. These signs are known as "biosignatures" - life's global impacts on the atmosphere and/or surface of a planetary environment.

The most useful biosignatures meet three criteria: reliability, survivability, and detectability, which together enhance the probability that the biosignature can be detected and interpreted as being due to life (e.g., Domagal-Goldman et al., 2011; Seager et al., 2012; Meadows, 2017). The reliability criterion seeks to answer whether an observed feature of the planetary environment has a biological origin, and whether it is more likely to be produced by life than by planetary processes such as geology and photochemistry. The survivability criterion determines whether the candidate biosignature gas can avoid the normal sinks in a planetary environment and build up to detectable levels. These sinks include, but are not limited to, destruction by photolysis and photochemistry, reactions with volcanic gases and the surface, and (for soluble gases) dissolution in the ocean. Finally, the detectability criterion asks whether the gas is spectrally active and clear of overlap with other chemical species in the wavelength region to be observed, and whether it is accessible in high enough abundance and at appropriate regions in the planetary atmosphere to be observed by techniques such as transmission, secondary eclipse, phase curves, and direct imaging.

With these three criteria, Earth's abundant molecular oxygen $\left(\mathrm{O}_{2}\right)$ has been identified as the strongest biosignature for terrestrial planets and was also initially thought to be straightforward to interpret as having a biological origin. $\mathrm{O}_{2}$ fulfills the three requirements of a biosignature in the following ways:

\subsection{Reliability}

It is the volatile byproduct of oxygenic photosynthesisthe metabolism driven by sunlight, which is the dominant source of energy on our planet's surface-and so clearly has a biological origin. Until recently, $\mathrm{O}_{2}$ was considered to have little or no abiotic planetary sources, as the Sun's UV spectrum drives photochemistry that produces only trace amounts in Earth's atmosphere (e.g., Walker, 1977; Hu et al., 2012; Domagal-Goldman et al., 2014; Harman et al., 2015). $\mathrm{O}_{2}$ also has no significant geological sources-unlike methane $\left(\mathrm{CH}_{4}\right)$ and carbon dioxide $\left(\mathrm{CO}_{2}\right)$, which are known to be produced by geological processes (Etiope and Sherwood-Lollar, 2013). Very early work considered $\mathrm{O}_{2}$ to be an even stronger biosignature when seen along with gases such as $\mathrm{CH}_{4}$ and nitrous oxide $\left(\mathrm{N}_{2} \mathrm{O}\right)$ in chemical thermodynamic disequilibrium (e.g., Lederberg, 1965; Lovelock, 1965; Hitchcock and Lovelock, 1967; Lovelock and Kaplan, 1975), although given Earth's negligible abiotic $\mathrm{O}_{2}$ sources, its high abundance alone indicates a biological origin.

\subsection{Survivability}

With a strong, planet-wide photosynthetic source, and relatively weak sinks compared with early periods of Earth's history (and different stellar hosts), $\mathrm{O}_{2}$ has risen over time to become the second most abundant gas in our atmosphere (at around $21 \%$ by volume). The atmosphere's more dominant gas, $\mathrm{N}_{2}$-which may also be biologically mediated (Catling and Kasting, 2007; Johnson and Goldblatt, 2015) - is considered a much poorer biosignature because $\mathrm{N}_{2}$ only exhibits strong absorption in the extreme ultraviolet (XUV; $0.01<\lambda$ $<0.121 \mu \mathrm{m})$. In this wavelength region, several other common molecules also absorb $\mathrm{H}_{2} \mathrm{O}, \mathrm{CO}_{2}, \mathrm{CO}, \mathrm{O}_{2}, \mathrm{CH}_{4}$ and other hydrocarbons (Rothman et al., 2013), and stars are much less luminous in the UV than in the visible regions, reducing the UV reflection or transmission signal from the planetary atmosphere. Sinks for $\mathrm{O}_{2}$ include outgassing of reduced gases such as $\mathrm{CH}_{4}$ and $\mathrm{H}_{2}$, crustal oxidation, and photolytic destruction in the stratosphere.

\subsection{Detectability}

These factors lead to a high abundance and even mixing throughout the atmospheric column, making $\mathrm{O}_{2}$ accessible to observation by transit spectroscopy. Depending on the observing geometry and atmospheric composition of a terrestrial (predominantly composed of silicate rocks and metals) planet, transmission spectroscopy may not reach the near-surface environment, instead probing the stratosphere and upper troposphere (Bétrémieux and Kaltenegger, 2013, 2014; Misra et al., 2014b; Meadows et al., 2018). This gives $\mathrm{O}_{2}$ a significant advantage over other proposed, often more complex biosignature molecules, including methanethiol and dimethyl disulfide (Pilcher, 2003; Domagal-Goldman et al., 2011; Seager and Bains, 2015), which are more susceptible to photolysis by UV radiation and so are confined largely to the lower troposphere, with significantly smaller concentrations in the stratosphere (Domagal-Goldman et al., 2011).

$\mathrm{O}_{2}$ is also one of the very few biogenic gases that absorbs strongly in the visible and near-infrared (NIR), likely wavelength regions to be covered by upcoming NASA missions and ground-based telescopes seeking to characterize extrasolar planets. $\mathrm{O}_{2}$ has strong features at wavelengths $<0.2 \mu \mathrm{m}$, and a $\gamma$ band at $0.628 \mu \mathrm{m}$, B-band at $0.688 \mu \mathrm{m}$, a strong A-band at $0.762 \mu \mathrm{m}$, and the $a^{1} \Delta_{\mathrm{g}}$ band at $1.269 \mu \mathrm{m}$ (Rothman et al., 2013). $\mathrm{O}_{2}$ could also be inferred from detection of its photochemical byproduct, ozone $\left(\mathrm{O}_{3}\right)$ (Ratner and Walker, 1972), which has strong bands in the UV $(0.2-0.3 \mu \mathrm{m})$, visible $(0.5-0.7 \mu \mathrm{m})$ and midinfrared (MIR) $(9.6 \mu \mathrm{m})$ (Rothman et al., 2013). $\mathrm{O}_{2}$ at high abundance could be identified by collisionally induced absorption as $\mathrm{O}_{2}$ molecules collide with each other or form the $\mathrm{O}_{4}$ complex, which has strong bands in the visible $(0.34$ $0.7 \mu \mathrm{m}$; Hermans et al., 1999; Thalman and Volkamer, 2013) and in the NIR (at $1.06 \mu \mathrm{m}$, Greenblatt et al., 1990; and at $1.27 \mu \mathrm{m}$, Maté et al., 2000).

However, although $\mathrm{O}_{2}$ is clearly a strong biosignature, recent research has indicated that its availability in a planetary atmosphere and its unique interpretation as a biological product may not be as straightforward as originally thought. These insights have come from exploration of the processes governing the long-term evolution of atmospheric $\mathrm{O}_{2}$ on the early Earth, and advances in theoretical modeling of star-planet interactions for planets orbiting other stars. This research indicates that the picture is far more complex, with both significant false negatives-planetary processes that suppress the buildup of atmospheric $\mathrm{O}_{2}$, despite the presence of biological production (Lyons et al., 2014; 
Planavsky et al., 2014b; Reinhard et al., 2017), and false positives - planetary processes that generate large (modern Earth-like) abundances of $\mathrm{O}_{2}$ abiotically (e.g., Wordsworth and Pierrehumbert, 2014; Luger and Barnes, 2015; Gao et al., 2015; Harman et al., 2015; Tian, 2015; see Meadows, 2017 for a review), being possible.

On the early Earth, it is likely that oxygenic photosynthesis was developed well in advance of the rise of $\mathrm{O}_{2}$ to significant levels in our atmosphere, constituting a false negative. Isotope measurements point to transient low levels of $\mathrm{O}_{2}$ in the early Earth's environment 3.0-2.65 Ga, which were likely generated by oxygenic photosynthesis (Czaja et al., 2012; Crowe et al., 2013; Planavsky et al., 2014a; Riding et al., 2014). Yet the irreversible, global accumulation of $\mathrm{O}_{2}$ in the atmosphere, which was likely mediated by burial and removal of organic carbon from Earth's surface environment (Kasting, 2001; Lyons et al., 2014), evidently occurred somewhat later, between 2.45 and 2.2 billion years ago (Ga; Farquhar, 2000; Bekker et al., 2004; Canfield, 2005). Recent sulfur isotope data (Luo et al., 2016) place this transition at $2.33 \mathrm{Ga}$. However, recent studies of Earth's carbon isotope record suggest that the record does not constrain the history of organic burial well enough to evaluate whether changes in fractional organic burial since $3.6 \mathrm{Ga}$ can explain the rise of $\mathrm{O}_{2}$ (Krissansen-Totton et al., 2015). Other proposed mechanisms for oxygen's rise include hydrogen escape from the upper atmosphere (Catling et al., 2001) and the long-term evolution of volcanic-tectonic processes at Earth's surface (Kump and Barley, 2007; Kasting, 2013; Lee et al., 2016). Additional data suggest that the permanent rise of $\mathrm{O}_{2}$ to high levels in Earth's atmosphere $(>1 \%$ of the present atmospheric level (PAL)) may have been delayed even further, to $0.8 \mathrm{Ga}$ (Planavsky et al., 2014b), long after the advent of oxygenic photosynthesis.

This delay between the evolution of the biological source of $\mathrm{O}_{2}$, photosynthesis, and the subsequent rise of abundant $\mathrm{O}_{2}$ in our atmosphere is due to diverse geological sinks for $\mathrm{O}_{2}$ in the environment effectively countering its biological production-ultimately resulting in a false negative. This example points to the importance of understanding a planetary environment to identify characteristics that may potentially reduce our ability to see a biosignature, even if a strong biological source exists.

Environmental context will also be extremely important for identifying the likelihood for false positives in a planetary environment-abiotic processes that could mimic a biosignature. $\mathrm{O}_{2}$ was long considered the most robust biosignature possible, because in Earth's modern environment, there were no known false positives, such as geological processes and photochemistry, that could generate it. For example, on Earth, the abiotic production of $\mathrm{O}_{2}$, principally by photolysis of water vapor, is at least a million times less than that produced by photosynthesis (Walker, 1977; Harman et al., 2015).

However, recent modeling studies examining star-planet interactions, especially for exoplanets orbiting $\mathbf{M}$ dwarfs, have identified several mechanisms that could potentially lead to the abiotic production of significant amounts of $\mathrm{O}_{2}$. These mechanisms ultimately rely on conditions that lead to the photolysis of $\mathrm{CO}_{2}$ or $\mathrm{H}_{2} \mathrm{O}$, two O-bearing gases that are likely to be common on terrestrial planets in the habitable zone (HZ). One mechanism for $\mathrm{O}_{2}$ buildup requires the vaporization of oceans, and the subsequent photolysis of water vapor and loss of $\mathrm{H}$, for planets orbiting early, superluminous M dwarf stars (Luger and Barnes, 2015). A low noncondensable gas inventory in a planetary atmosphere can drive high stratospheric $\mathrm{H}_{2} \mathrm{O}$ abundances by increasing the altitude of the tropospheric "cold trap," leading to the photolysis of $\mathrm{H}_{2} \mathrm{O}$ and the loss of $\mathrm{H}$ to space. Because the water loss is a strong function of the planet's atmospheric composition, this potential abiotic $\mathrm{O}_{2}$ generation mechanism can affect planets orbiting any type of star (Wordsworth and Pierrehumbert, 2014). Finally, photolysis of $\mathrm{CO}_{2}$ as a source of atmospheric $\mathrm{O}_{2}$ may occur on planets orbiting $\mathrm{M}$ dwarfs. Whether photolytic $\mathrm{O}_{2}$ is produced is dependent on both how well the UV spectrum of the star photolyzes $\mathrm{CO}_{2}$ and the suppression of catalytic or other processes that would allow the $\mathrm{CO}$ and $\mathrm{O}$ to rapidly recombine (e.g., DomagalGoldman et al., 2014; Tian et al., 2014; Gao et al., 2015; Harman et al., 2015). Each of these mechanisms leaves telltale signs in the planetary environment that can point to the abiotic source of the $\mathrm{O}_{2}$ (e.g., Schwieterman et al., 2015b, 2016).

Rather than demonstrating that $\mathrm{O}_{2}$ is not suitable as a biosignature, our new and evolving understanding of false positives for $\mathrm{O}_{2}$ increases its robustness (Meadows, 2017). The study of $\mathrm{O}_{2}$ as a biosignature has taught us that by attempting to understand the environmental context, including the possibility for false positives and negatives, we will ultimately improve our interpretation of exoplanet observations and increase our confidence that they do indeed indicate the presence of life. Knowledge gained from the delayed rise of $\mathrm{O}_{2}$ during the history of our own planet helps us identify environmental contexts for false negatives for $\mathrm{O}_{2}$, and interdisciplinary modeling studies of exoplanets have helped us to explore and identify the stellar and planetary characteristics that are more likely to lead to $\mathrm{O}_{2}$ false negatives. This knowledge can guide target selection for habitable planets, and can be used to design observations to search for the necessary environmental context to increase our confidence in the interpretation. Moreover, these studies serve as a template for future exoplanet biosignature development, and the lessons learned from our study of $\mathrm{O}_{2}$ can help guide the development of a generalized framework for biosignature development and detection (see e.g., Catling et al., 2018).

In Section 2, we describe in more detail the evolution of $\mathrm{O}_{2}$ throughout Earth's history, highlighting the ways in which the environment initially suppressed and then finally permitted its development as a detectable biosignature. In Section 3, we discuss the known potential false positive mechanisms for terrestrial exoplanets, and their likely observable environmental impact. In Section 4, we discuss the specific observational implementation for $\mathrm{O}_{2}$ detection and how confidence in its biogenicity can be enhanced, including (1) characterization of planetary, stellar, and planetary system environmental context; (2) the search for $\mathrm{O}_{2}$; and (3) the specific environmental characteristics that could be sought to help discriminate false negatives and positives. We describe the photometric and spectroscopic measurements required to execute these three steps for different near-term exoplanet observing facilities, including the James Webb Space Telescope (JWST), extremely large ground-based telescopes, and for proposed space-based direct imaging coronographic/starshade missions. In Section 
5, we discuss and prioritize the most important discriminating measurements, and then discuss the robustness of any detection as a function of accessible wavelength range and other telescope characteristics. In Section 6, we present our conclusions.

\section{The Coevolution of Life with Its Environment, False Negatives, and the Rise of Earth's $\mathrm{O}_{2}$}

There are tantalizing suggestions that life may have emerged very early during the 4.6 billion year evolution of our planet, but the development of photosynthesis, and the subsequent oxygenation of the atmosphere, likely occurred much later. Carbonaceous compounds enriched in ${ }^{12} \mathrm{C}$ (Mojzsis et al., 1996; Rosing, 1999), potentially biogenic sedimentary structures (Nutman et al., 2016), and purported microfossils (Dodd et al., 2017) are present in the oldest sedimentary rocks on Earth. Furthermore, ${ }^{12} \mathrm{C}$-enriched graphitic inclusions in recycled zircons have been suggested to date the emergence of life to before the earliest sedimentary rocks, at $\sim 4.1 \mathrm{Ga}$ (Bell et al., 2015). These observations are consistent with a biological origin, but in all cases are somewhat equivocal (see, for example, Lepland et al., 2002; van Zuilen et al., 2002; McCollom and Seewald, 2006). Nevertheless, strong isotopic evidence for the presence of important microbial metabolisms such as methanogenesis (Ueno et al., 2006) and dissimilatory sulfate reduction (Shen et al., 2001; Ueno et al., 2009) is observable by at least $\sim 3.5 \mathrm{Ga}$, suggesting that although the precise timing of biochemical steps remains to be elucidated, key landmarks in evolution of life - the emergence of life from prebiotic chemistry, the development of cellularity, and the development of metabolism-likely occurred relatively soon after Earth's formation.

Photosynthesis, the ability to harvest the Sun's energy to support life, may have also evolved early in our planet's history, with multiple geological and geochemical lines of evidence suggesting that anoxygenic phototrophs were present on Earth by at least 3.3 Ga (Westall et al., 2011) and possibly as far back as 3.5-3.7 Ga (Buick, 1981; Nutman et al., 2016). These early anoxygenic phototrophs used strong reductants such as hydrogen or hydrogen sulfide to donate electrons to a single photosystem (PSI) that harvested light (Olson and Pierson, 1986). Volcanism could have produced these reductants at sufficient concentrations to drive photosynthesis, but only in relatively limited environments. Consequently early Earth's anoxygenic phototrophs would have been challenging to detect remotely, as their productivity would have been limited by the spatial restriction of their reductants, with a resultant relatively small impact on Earth's globally averaged characteristics (e.g., Canfield et al., 2006). These phototrophs are also not known to produce gaseous byproducts.

The evolution of oxygenic photosynthesis to use globally abundant water was arguably one of the most important biological innovations on Earth. Oxygenic photosynthesis uses energy from the Sun to convert $\mathrm{CO}_{2}$ and liquid water to glucose, with $\mathrm{O}_{2}$ as a byproduct:

$$
6 \mathrm{CO}_{2}+6 \mathrm{H}_{2} \mathrm{O} \rightarrow \mathrm{C}_{6} \mathrm{H}_{12} \mathrm{O}_{6}+6 \mathrm{O}_{2}
$$

There are competing theories on the antiquity of oxygenic photosynthesis, and more specifically water oxidation, as some researchers believe that this metabolic capability may have arisen very soon after the origin of life (e.g., Cardona, 2015, 2016, 2017). However, recent phylogenetic analyses examining the origins of oxygenic photosynthesis in cyanobacteria have found that microbes basal to the cyanobacterial lineage (Melainabacteria and Sericytochromatia) lack photosynthetic machinery. This suggests that photosynthesis arose late in cyanobacteria, and that these genes were acquired by horizontal gene transfer from more primitive anoxygenic phototrophs (Soo et al., 2017).

Because $\mathrm{H}_{2} \mathrm{O}, \mathrm{CO}_{2}$, and sunlight are globally available components of the habitable terrestrial environment, they allow oxygenic photosynthesis to dominate large fractions of the planetary surface (Kiang et al., 2007a; Léger et al., 2011). The subsequent oxygenation of the atmosphere and oceans significantly impacted Earth's global environment, and ultimately allowed for biological (Knoll et al., 2006) and mineralogical diversification (Hazen et al., 2008). Oxygenic photosynthesis maintains much of the living biomass on our planet today (Falkowski et al., 2000), and its dominance in our biosphere and its global distribution have also made photosynthesis more detectable on a planetary scale. However, whether $\mathrm{O}_{2}$ rises to detectable levels in a planetary atmosphere will depend on the balance between sources and sinks for $\mathrm{O}_{2}$ in surface environments, and in particular whether planetary processes, such as geological burial of organic carbon (e.g., Des Marais et al., 1992), atmospheric escape of hydrogen (Catling et al., 2001), or changes in reductant fluxes to the surface (Kasting, 2013) can support the buildup of biologically generated $\mathrm{O}_{2}$ in the atmosphere on long timescales.

In this section, we discuss the evolution of oxygenic photosynthesis in an environmental and geochemical context, including the suppression and eventual rise of atmospheric $\mathrm{O}_{2}$, and the interplay between biological production of $\mathrm{O}_{2}$ and its consumption by sinks present in early Earth surface environments. Ultimately, we wish to understand the environmental selective pressures driving the evolution of water oxidation and $\mathrm{O}_{2}$ production on the early Earth, so they can be generalized and extrapolated to rocky habitable exoplanets.

\subsection{The evolution of oxygenic photosynthesis}

Much research has been directed at understanding the evolutionary transition between anoxygenic and oxygenic photosynthesis, and this article is not intended to be an exhaustive review of the various schools of thought. There is a difference in the complexity of photosynthetic machinery between oxygenic and anoxygenic phototrophs. The light reactions of oxygenic photosynthesis in cyanobacteria, algae, and plants use two linked photosystems: photosystems I (PSI) and II (PSII). PSII and the oxygen-evolving complex allow for the extraction of electrons from water molecules, which have a very positive redox potential $\left(\mathrm{O}_{2} / \mathrm{H}_{2} \mathrm{O}\right.$ pair; $E_{0}{ }^{\prime}=+0.87 \mathrm{~V}$ ) (see overview in Schwieterman et al., 2018). The photosynthetic machinery of anoxygenic phototrophs is different. These primitive phototrophs use a single photosystem, which can be classified into one of two families-type I reaction centers, including PSI in chloroplasts, cyanobacteria, green sulfur bacteria, heliobacteria, and phototrophic acidobacteria, and type II reaction centers, such as PSII in 
chloroplasts, cyanobacteria, purple bacteria, and green nonsulfur bacteria (Allen and Williams, 1998; Bryant et al., 2007). Rather than using chlorophyll pigments that absorb light at wavelengths in the visible range, anoxygenic phototrophs utilize a diversity of bacteriochlorophyll pigments that absorb in the NIR (Senge and Smith, 1995).

The evolution of photosynthesis may have been driven, in part, by the redox potential of electron donors or reductants within an environmental context, with a step-wise increase in the redox potentials of reductants donating electrons to PSII (Pierson and Olson, 1989; Olson, 2006). The most ancient form of anoxygenic photosynthesis uses strong reductants such as $\mathrm{H}_{2}$ or $\mathrm{H}_{2} \mathrm{~S}$ to donate electrons to a single photosystem (Olson and Pierson, 1986), but the limited availability of these volcanogenic compounds may have provided evolutionary pressures to develop a second photosystem, which could use weaker but more abundant reductants, such as Fe(II) and water (Olson, 1970, 1978).

Modern cyanobacteria produce $\mathrm{O}_{2}$ resulting from the extraction of electrons from water, but there is a large difference in the redox potentials between the $\mathrm{O}_{2} / \mathrm{H}_{2} \mathrm{O}$ pair $\left(E_{0}{ }^{\prime}=+0.87 \mathrm{~V}\right)$ of oxygenic photosynthesis and the $\mathrm{S}_{0} / \mathrm{HS}^{-}$ pair $\left(E_{0}{ }^{\prime}=-0.27 \mathrm{~V}\right)$ commonly used by anoxygenic photosynthesis. Researchers have speculated that an intermediate reductant, such as $\mathrm{Fe}(\mathrm{II})\left(E_{0}{ }^{\prime}=+0.30 \mathrm{~V}\right.$ at circumneutral $\mathrm{pH})$ or $\mathrm{Mn}(\mathrm{II})\left(E_{0}{ }^{\prime}=\sim+0.60 \mathrm{~V}\right)$, could have bridged the gap and acted as a transitional electron donor before water (Pierson and Olson, 1989; Olson, 2006). The widespread abundance of reduced iron (and potentially reduced manganese) on the early Earth would have made it particularly suitable as an electron donor for photosynthesis. Pierson et al. (1993) pointed out that the oxidized iron products of this type of photosynthesis would have provided substantial protection from UV radiation for surface-dwelling phototrophs before the development of an $\mathrm{O}_{3}$ shield.

Due, in part, to the wide availability of water as an electron donor compared with the limited availability of volcanogenic reductants, oxygenic phototrophs spread easily and dominated aquatic and terrestrial habitats. Indeed, the evolution of oxygenic photosynthesis would ultimately result in a radical restructuring of Earth surface environments, with significant impacts on all major biogeochemical cycles. The so-called Great Oxidation Event, which occurred at $\sim 2.3 \mathrm{Ga}$ (Bekker et al., 2004; Luo et al., 2016), set the stage for the evolution of more complex life-forms dependent on $\mathrm{O}_{2}$. Consequently, $\mathrm{O}_{2}$ is not only a promising biosignature, but it may also indicate an environment in which multicellularity, and even intelligence, can be supported (e.g., Catling et al., 2005).

It is of course difficult to estimate the likelihood that oxygenic photosynthesis would evolve on an exoplanet. The availability of carbon sources $\left(\mathrm{CO}_{2}\right.$ and/or reduced carbon) must be considered, and in the case of $\mathrm{CO}_{2}$ fixation, the availability of reductants or electrons must also be factored in. In speculating about the evolution of photoautotrophy on an exoplanet, volcanism-a common geological process on terrestrial habitable planets-can supply $\mathrm{CO}_{2}$ and other reduced species such as $\mathrm{H}_{2}$. The possibility may exist that the same evolutionary selective pressures based on electron donor availability that gave rise to oxygenic photosynthesis on the early Earth could play out on an exoplanet, leading to the same metabolic outcome.
If photosynthesis did evolve on an exoplanet, it would likely coevolve with its environment to develop lightharvesting pigments that make use of the available stellar spectrum at the surface of the planet. Previous work suggested that chlorphyll $a$ evolved to absorb the peak solar photon flux onto the planetary surface (Kiang et al., 2007a, 2007b), which could potentially be predicted for different star-planet combinations. The peak photon flux will be the result of the interplay between the incident solar spectrum and subsequent absorption by molecules in the atmosphere, as well as efficiency considerations for NIR radiation, which may be too low in energy to drive photosynthesis efficiently beyond a limiting wavelength (Kiang et al., 2007a, 2007b). On Earth, $\mathrm{O}_{3}$ absorption in our atmosphere drives the Sun's incident peak photon flux near $600 \mathrm{~nm}$ redward. The peak photon flux at the surface is at $688 \mathrm{~nm}$, where the principal photosynthetic pigment, chlorophyll $a$, absorbs (Kiang et al., 2007b). However, on the early Earth, without abundant $\mathrm{O}_{3}$, the peak photon flux at the surface may have been different, but potentially also pushed redward by strong blue absorption from hydrocarbon haze. Such hazes may have been present during early periods of Earth's history when the atmosphere was more reducing (Arney et al., 2016).

In the case of exoplanets, the spectrum of the host star will be well known in the visible range, and the planetary atmospheric composition may be constrained by spectral observations. These observations will allow radiative transfer models to predict photon fluxes at the planetary surface, and potentially help identify the most likely spectral region for photosynthetic pigment absorption. However, it should be noted that the Kiang et al. (2007a, 2007b) supposition that phototrophs evolved to absorb the maximum photon flux (which was developed when considering vegetation) does not hold up when applied to cyanobacteria and anoxygenic phototrophs. Photosynthesis in these microbes is often inhibited by high light levels, and maximum photosynthetic activity can sometimes be observed at only 10$20 \%$ of the maximum solar irradiation (e.g., Murata et al., 2007; Trouwborst et al., 2007).

\subsection{The suppression, and ultimate rise, of $\mathrm{O}_{2}$ on the early Earth}

Although the evolution of oxygenic photosynthesis represented a critical evolutionary innovation, it was not a sufficient condition for appreciable and persistent planetary oxygenation, which may not have happened until as recently as $0.8 \mathrm{Ga}$ (Lyons et al., 2014). Understanding oxygen's late rise in our atmosphere requires a basic discussion of the factors modulating Earth's global $\mathrm{O}_{2}$ cycle (Fig. 1). Oxygenic photosynthesis on the modern Earth fixes carbon (i.e., converts atmospheric $\mathrm{CO}_{2}$ into organic carbon) at a net rate of $\sim 100 \mathrm{Pg}\left(10^{15} \mathrm{~g}\right)$ of carbon per year, distributed roughly evenly between the marine and terrestrial photosynthetic biospheres (Field et al., 1998). For every mole of carbon fixed into biomass by oxygenic photosynthesis, one mole of free $\mathrm{O}_{2}$ is released to the environment.

However, the vast majority of this $\mathrm{O}_{2}$ is consumed very rapidly through aerobic respiration in surface environments, with only a very small fraction of the organic carbon initially produced by oxygenic photosynthesis (less than 1\%) escaping respiration and becoming buried in marine and 

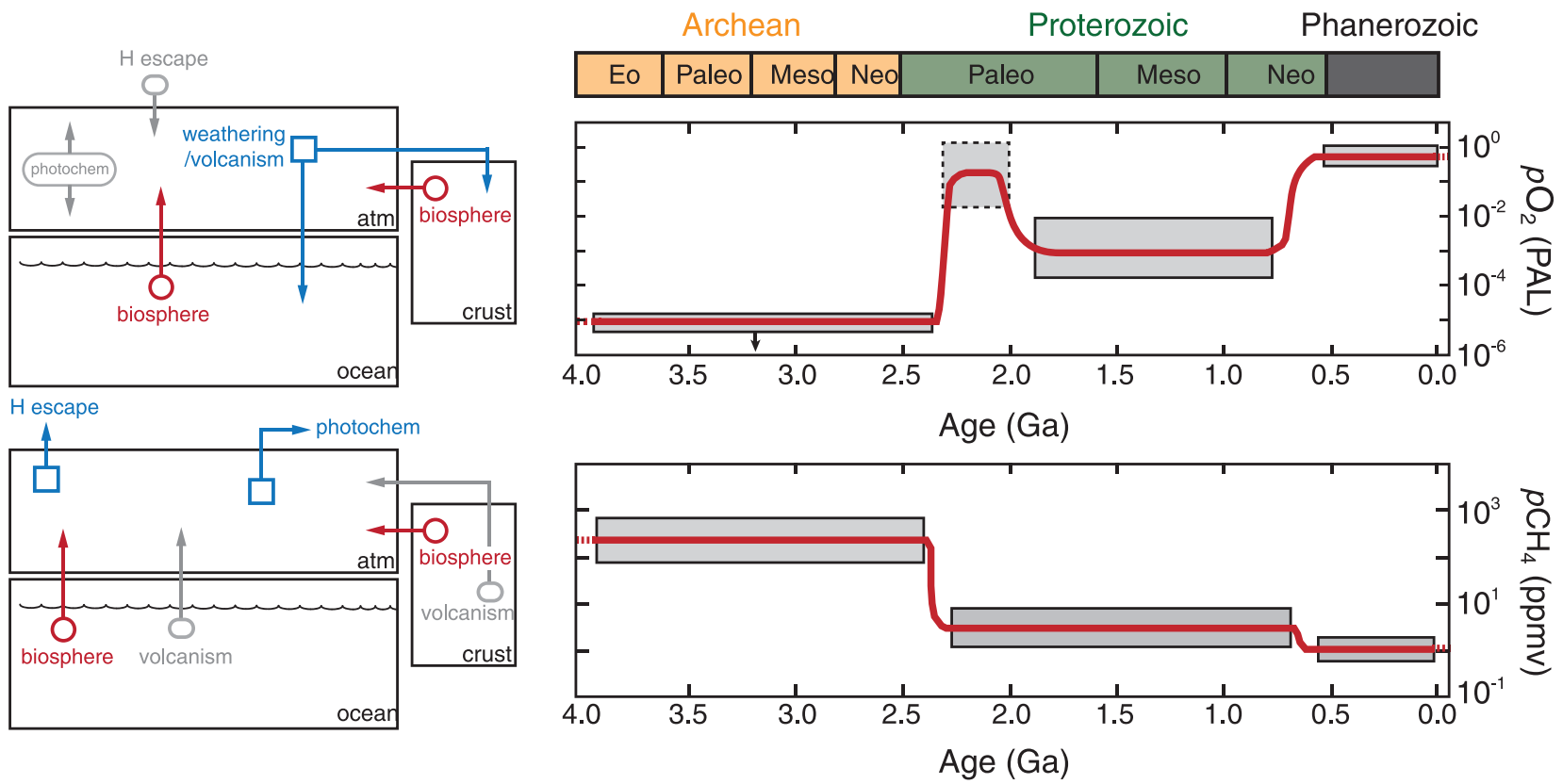

FIG. 1. Overview of the major processes controlling atmospheric $\mathrm{O}_{2}$ and $\mathrm{CH}_{4}$ levels (left panels). Red circles/arrows denote net biogenic sources (e.g., after accounting for biological consumption), gray ovals/arrows denote potential abiotic sources, and blue squares/arrows denote net sink fluxes. Schematic depiction of the evolution of atmospheric $\mathrm{O}_{2}$ and $\mathrm{CH}_{4}$ throughout Earth's history (right panels). Using results from Reinhard et al. (2017), shaded boxes show ranges based on geochemical proxy or model reconstructions, whereas red curves show possible temporal trajectories through time. (Credit: Chris Reinhard)

terrestrial sediments (Berner, 1982). This relatively slow "leak" of organic carbon into Earth's upper crust represents a net source of $\mathrm{O}_{2}$ to Earth's atmosphere. In addition, a fraction of photosynthetically produced organic matter that escapes burial will be consumed by microbial sulfate reduction, which essentially transfers electrons from organic matter to sedimentary pyrite $\left(\mathrm{FeS}_{2}\right)$, the burial of which similarly results in a net release of $\mathrm{O}_{2}$ to surface environments. These fluxes are augmented by the irreversible escape of $\mathrm{H}$ to space, which scales with the total abundance of $\mathrm{H}$-bearing reduced gases at the homopause (e.g., $\mathrm{CH}_{4}$ and $\mathrm{H}_{2}$; Hunten, 1973). Although this represents a very small flux on the modern Earth, it is critical to consider as a mechanism of secular oxidation on timescales of planetary evolution (Catling et al., 2001). Input fluxes of $\mathrm{O}_{2}$ are ultimately balanced on long timescales by $\mathrm{O}_{2}$ consumption during the uplift and oxidative weathering of reduced mineral phases in Earth's crust (including previously buried organic carbon and pyrite sulfur) and through reaction with volcanic and metamorphic reductants (Kasting and Canfield, 2012).

Whether a planet develops a biogenic $\mathrm{O}_{2}$-rich atmosphere will thus depend not only on the evolution of oxygenic photosynthesis but also on the long-term balance between sources and sinks of $\mathrm{O}_{2}$ at the surface (e.g., Gebauer et al., 2017). The accumulation and stability of large biogenic $\mathrm{O}_{2}$ inventories in a planetary atmosphere will, in turn, depend on how input/output fluxes scale with atmospheric $\mathrm{O}_{2}$ levels (if at all) and on secular changes in planetary degassing, differentiation, and atmospheric escape. An important consequence of this is that the evolution of oxygenic photosynthesis cannot, on its own, be expected to yield a remotely detectable oxidizing atmosphere. The planetary environment also plays a crucial role.

Earth's history provides an instructive example. Constraints from stable isotopes and trace element proxies indicate the evolution of oxygenic photosynthesis on Earth by at least $\sim 3.0 \mathrm{Ga}$ (Planavsky et al., 2014a) and perhaps much earlier (Rosing and Frei, 2004). However, complementary isotopic constraints indicate that Earth's atmosphere was pervasively reducing until $\sim 2.5 \mathrm{Ga}$ (Farquhar et al., 2001; Pavlov and Kasting, 2002; Zahnle et al., 2006). There thus appears to have been a very significant period on Earth during which oxygenic photosynthesis was present but large amounts of $\mathrm{O}_{2}$ did not accumulate in Earth's atmosphere. In addition, there is some evidence that after the initial accumulation of $\mathrm{O}_{2}$ in Earth's atmosphere at $\sim 2.3 \mathrm{Ga}$, atmospheric $\mathrm{O}_{2}$ levels remained relatively low for much of the subsequent $\sim 2$ billion years (Planavsky et al., 2014b; Cole et al., 2016; Tang et al., 2016), during which biogenic $\mathrm{O}_{2}$, although clearly present, may have been challenging to detect remotely given current technology (Reinhard et al., 2017).

In sum, the emergence of a biogenic $\mathrm{O}_{2}$-rich atmosphere will depend on both the evolution of oxygenic photosynthesis and geochemical dynamics at the planetary surface that are favorable for the long-term accumulation of a large atmospheric $\mathrm{O}_{2}$ inventory; if planetary conditions are not favorable, then a false negative will occur. These dynamics will, in turn, depend on a series of planetary factors that may be challenging to constrain observationally or from first principles. For example, heat flux from a planetary interior (as constrained by radiogenic element inventory and planet size), $\mathrm{O}_{2}$ fugacity of the planetary mantle (as constrained by 
both initial chemistry and long-term recycling of materials from the surface), the degree of crustal differentiation (as constrained by both overall heat fluxes and planetary rheology), and ocean chemistry can interact to buffer atmospheric $\mathrm{O}_{2}$ to low levels despite the presence of oxygenic photosynthesis. The ability to constrain these contextual variables through observations of the planet and star, or through modeling, may ultimately form a critical component of diagnosing false negatives for $\mathrm{O}_{2}$ on living planets. Similarly, they lay the foundation for critical thinking on the nature and use of contextual information for other proposed biosignatures as well.

\subsection{Earth's history of oxygen's companion biosignatures $\mathrm{CH}_{4}$ and $\mathrm{N}_{2} \mathrm{O}$}

The role of the environment in enhancing or suppressing the atmospheric accumulation of biogenic gases also applies to other species, such as $\mathrm{CH}_{4}$ and $\mathrm{N}_{2} \mathrm{O}$, and the detection of these gases could be used to strengthen confidence that the observed $\mathrm{O}_{2}$ in an exoplanet atmosphere is indeed biogenic (e.g., Hitchcock and Lovelock, 1967). Atmospheric levels of these gases will be a function of production at the surface (whether biological or abiotic), but will also depend strongly on sink fluxes due to biological recycling, geological processes, and photochemistry in the atmosphere. For example, $\mathrm{N}_{2} \mathrm{O}$ is photochemically unstable in reducing atmospheres (Roberson et al., 2011), as these atmospheres lack the $\mathrm{O}_{2}$, $\mathrm{O}_{3}$, or $\mathrm{CO}_{2}$ of more oxidizing atmospheres, which shields $\mathrm{N}_{2} \mathrm{O}$ from UV photolysis to $\mathrm{N}_{2}$. Consequently, in more reducing atmospheres, it is difficult to sustain high atmospheric levels of $\mathrm{N}_{2} \mathrm{O}$ without large surface fluxes. The abundance of $\mathrm{N}_{2} \mathrm{O}$ in planetary atmospheres also depends on the spectrum of the host star, and many $M$ dwarfs are less able to directly photolyze $\mathrm{N}_{2} \mathrm{O}$, allowing longer lifetimes and higher abundances for the same surface flux (Segura et al., 2005; Rauer et al., 2011; Rugheimer et al., 2013, 2015). However, in general, unless the atmosphere is already oxidizing, whether due to the action of oxygenic photosynthesis, or stellar-driven atmospheric evolution to high- $\mathrm{O}_{2}$ or high- $\mathrm{CO}_{2}$ states (e.g., Meadows et al., 2018; Luger and Barnes, 2015), relatively high $\mathrm{N}_{2} \mathrm{O}$ fluxes will be required to maintain elevated atmospheric $\mathrm{N}_{2} \mathrm{O}$ levels. These kinetic constraints on biogenic gas abundance in planetary atmospheres have led to approaches that emphasize the magnitude of chemical disequilibrium between species (e.g., $\mathrm{O}_{2} / \mathrm{O}_{3}$ and $\left.\mathrm{CH}_{4}\right)$, and this approach can potentially provide more specific information about the magnitude of production/consumption fluxes compared with the abundance of a single biogenic gas (Lovelock and Kaplan, 1975; Kasting et al., 2013; Krissansen-Totton et al., 2016a).

The evolution of atmospheric chemistry on Earth, as described hereunder, illustrates how searching for secondary gases to strengthen confidence in oxygen's biogenic origin may be both challenging and illuminating (Fig. 3).

2.3.1. The Archean $(4.0-2.5 \mathrm{Ga})$-low $\mathrm{O}_{2}$, high $\mathrm{CH}_{4}$. During most of the Archean Eon, background atmospheric $\mathrm{O}_{2}$ levels were vanishingly low (likely less than $\sim 10^{-7}$ times PAL; Zahnle et al., 2006), with peak $\mathrm{O}_{3}$ levels correspondingly low (Kasting and Donahue, 1980). Theoretical models predict that under such reducing atmospheric conditions, and with relatively low oxidant levels in the ocean (e.g., dissolved $\mathrm{O}_{2}, \mathrm{NO}_{3}{ }^{-}$, and $\mathrm{SO}_{4}{ }^{2-}$ ), atmospheric $\mathrm{CH}_{4}$ levels may have been $\sim 2-3$ orders of magnitude above those of the modern Earth (Fig. 3; Pavlov et al., 2003). Indeed, new isotopic tools are emerging for fingerprinting the photochemical impacts of much higher atmospheric $p \mathrm{CH}_{4}$ and its modulation by Earth's biosphere (e.g., Izon et al., 2017). Although there is now firm evidence for transient increases in atmospheric $\mathrm{O}_{2}$ levels during the late Archean (Anbar et al., 2007; Kendall et al., 2010), the magnitude and duration of these $\mathrm{O}_{2}$ pulses are not well constrained at present. Thus, for much of Archean time, Earth's atmosphere was characterized by very low $\mathrm{O}_{2} / \mathrm{O}_{3}$ but elevated $\mathrm{CH}_{4}$.

2.3.2. The Paleoproterozoic $(\sim 2.2-2.0 \mathrm{Ga})$-higher $\mathrm{O}_{2}$, low $\mathrm{CH}_{4}$, possible $\mathrm{N}_{2} \mathrm{O}$. After the first large-scale oxygenation of the ocean-atmosphere system after $\sim 2.3 \mathrm{Ga}$, there was a period of the Paleoproterozoic during which atmospheric $\mathrm{O}_{2} / \mathrm{O}_{3}$ levels may have risen to (or even exceeded) modern levels for perhaps 100-200 million years or more (Fig. 3; Lyons et al., 2014). This increase in atmospheric $\mathrm{O}_{2}$ would, in all likelihood, have led to a corresponding drop in atmospheric $\mathrm{CH}_{4}$ levels, and may have UV shielded and photochemically stabilized $\mathrm{N}_{2} \mathrm{O}$ for the first time in Earth's history.

2.3.3. The mid-Proterozoic $(\sim 1.8-0.8 \mathrm{Ga})$-lower $\mathrm{O}_{2}$, low $\mathrm{CH}_{4}$. Atmospheric $\mathrm{O}_{2} / \mathrm{O}_{3}$ levels appear to have dropped markedly and remained at relatively low background levels for much of the mid-Proterozoic (Lyons et al., 2014; Planavsky et al., 2014b; Cole et al., 2016; Reinhard et al., 2016; Tang et al., 2016). At the same time, recent Earth system modeling of the $\mathrm{CH}_{4}$ cycle under mid-Proterozoic conditions as inferred from the geochemical proxy record (e.g., atmospheric $\mathrm{pO}_{2}$ and marine $\mathrm{SO}_{4}{ }^{2-}$ levels) implies that atmospheric $\mathrm{CH}_{4}$ may also have been rather low, on the order of $\sim 1-10$ ppmv (Olson et al., 2016).

2.3.4. The late Proterozoic ( $800-550$ million years ago [Ma])-higher $\mathrm{O}_{2}$, low $\mathrm{CH}_{4}$, higher $\mathrm{N}_{2} \mathrm{O}$. The geochemical record indicates another series of significant shifts in oceanatmosphere redox during the late Proterozoic, after which atmospheric $\mathrm{O}_{2} / \mathrm{O}_{3}$ levels may have ultimately stabilized at roughly modern levels. Atmospheric $p \mathrm{O}_{2}$ subsequently varied between $\sim 0.25$ and 1.5 PAL over the past $\sim 500$ million years (Berner et al., 2006). Earth's atmosphere during this period has been characterized by correspondingly low atmospheric $\mathrm{CH}_{4}$ (Fig. 3) and possibly relatively high $\mathrm{N}_{2} \mathrm{O}$ production (Buick, 2007) and atmospheric $\mathrm{N}_{2} \mathrm{O}$.

One striking implication of this history is that throughout Earth's evolution, either $\mathrm{O}_{2}$ or $\mathrm{CH}_{4}$, or neither, dominated the atmosphere, and so the canonical $\mathrm{O}_{2}-\mathrm{CH}_{4}$ disequilibrium biosignature may have been challenging to observe in the atmosphere over most of Earth's history. This is despite the fact that oxygenic photosynthesis has been present at Earth's surface for at least $\sim 3$ billion years (Planavsky et al., 2014a), and methanogenesis has been extant for $\sim 3.5$ billion years (Ueno et al., 2006). The source-sink relationships for the coupled $\mathrm{O}_{2}$ and $\mathrm{CH}_{4}$ cycles throughout Earth's history may thus have led to a persistent false negative signal, during which major biogenic gases were being 
produced and consumed at potentially high rates, but did not coaccumulate in the atmosphere at potentially detectable levels (Reinhard et al., 2017). Possible exceptions include relatively high $\mathrm{O}_{2} / \mathrm{O}_{3}$ during the Paleoproterozoic and during the past $\sim 500$ million years, and potentially detectable $\mathrm{O}_{3}$ during the mid-Proterozoic (Reinhard et al., 2017). However, the possibility remains that Earth's surface biosphere, definitively established by at least $\sim 3.5$ billion years ago, may not have been detectable by using biosignature gases until relatively recently in Earth's geological history.

\subsection{Secondary photosynthetic biosignatures}

Earth also demonstrates alternative ways in which our photosynthetic biosphere has impacted the environment beyond the abundant $\mathrm{O}_{2}$ that eventually rose in our atmosphere. These secondary biosignatures include surface reflectivity signatures from light harvesting (Kiang et al., 2007a, 2007b) and other pigments developed for nonphotosynthetic purposes by phototrophs (Hegde et al., 2015; Schwieterman et al., 2015a), as well as the strong jump in reflectivity longward of chlorophyll $a$ at $0.7 \mu \mathrm{m}$, from the red edge of vegetation (Gates et al., 1965; Kiang et al., 2007a). Another secondary biosignature of photosynthesis in globally averaged spectra of our planet is the seasonal variations in the abundance of $\mathrm{CO}_{2}$ (Meadows, 2008) due to growth and decay of vegetation on land, which imparts a 2\% seasonal variation in $\mathrm{CO}_{2}$ abundance (Keeling, 1960; Hall et al., 1976; Keeling et al., 1976). Earlier in Earth's history before the permanent rise of $\mathrm{O}_{2}$, small amounts of biogenic $\mathrm{O}_{2}$ may also have shown seasonal variation (see Schwieterman et al., 2018, for a review; Reinhard et al., 2016).

Either a corroborative surface or temporal signature may be sought as a secondary confirmation of a photosynthetic origin for any detected $\mathrm{O}_{2}$ in a planetary atmosphere. The vegetation red edge has likely been widespread on Earth since about $0.46 \mathrm{Ga}$ (Carroll, 2001; Igamberdiev and Lea, 2006), corresponding with the rise of land plants, and may be coincident with the rise of $\mathrm{O}_{2}$ to near-modern levels. However, in false negative situations wherein planetary processes are suppressing the rise of photosynthetically generated $\mathrm{O}_{2}$, surface reflectivity biosignatures from photosynthetic and nonphotosynthetic pigments produced by phototrophs may have been the only way to detect photosynthesis (Schwieterman et al., 2015a).

\section{Star-Planet Interactions, and the Generation of False Positives for $\mathrm{O}_{2}$ in Exoplanetary Environments}

Although false negatives may reduce the atmospheric signal and so preclude the detectability of biosignatures, false positives complicate the interpretation of any $\mathrm{O}_{2}$ that is observed. False positives for $\mathrm{O}_{2}$ on uninhabitable planets have been postulated for decades (Schindler and Kasting, 2000; Selsis et al., 2002; Des Marais et al., 2002 and references therein), but were considered more likely to occur for planets outside the habitable zone, and so were anticipated to be easy to identify and exclude. However, several plausible $\mathrm{O}_{2}$ false positive scenarios for planets in the habitable zone have since been identified (e.g., Wordsworth and Pierrehumbert, 2014; Luger and Barnes, 2015; Tian, 2015; Harman et al., 2015; see Meadows, 2017 for a more detailed review). These mechanisms rely primarily on conditions that lead to the photolysis of $\mathrm{H}_{2} \mathrm{O}$ and/or $\mathrm{CO}_{2}$ in a terrestrial planet atmosphere, and in some cases stabilize the photolytic byproducts of $\mathrm{CO}_{2}$ from recombination by suppressing the photolytic formation of catalysts from other atmospheric gases. Rather than weakening oxygen's role as a biosignature, knowledge of false positives allows us to identify observable characteristics of the stellar or planetary environment that indicate the mechanisms that most likely generate them. Each potential false positive mechanism that is ruled out by observations increases the robustness of a biogenic interpretation for $\mathrm{O}_{2}$. Hereunder we summarize some of the most significant false positive mechanisms for planets in the habitable zone.

\subsection{Low noncondensable gas inventories}

The possible abiotic $\mathrm{O}_{2}$ generation mechanism that will likely be the most difficult to observationally recognize and preclude involves photolysis of water-and subsequent hydrogen loss-from terrestrial atmospheres that are depleted in noncondensable gases such as $\mathrm{N}_{2}$ (Wordsworth and Pierrehumbert, 2014). This mechanism may produce Earth-like quantities of $\mathrm{O}_{2}$ on an ocean-bearing world. To work, the effectiveness of the "cold trap"-the rapid reduction in temperature with altitude that causes rising water vapor on Earth to condense and thereby remain trapped in the troposphere-must be weakened. This occurs when the atmospheric temperatures are high, or when the total inventory of noncondensable gases-for example, $\mathrm{N}_{2}, \mathrm{O}_{2}$, or $\mathrm{CO}_{2}$-is low (Wordsworth and Pierrehumbert, 2014). Because this mechanism to allow water into the stratosphere relies primarily on a planetary property, a low inventory of gases that do not condense at typical habitable zone terrestrial planetary temperatures and pressures, it may work for planets orbiting stars of any spectral type, including Sun-like stars. With a low inventory of noncondensable gases, water is able to rise higher into the atmosphere before it condenses, and it is more vulnerable to photolysis by incident stellar UV radiation at these higher altitudes. The water's $\mathrm{H}$ atoms escape, leaving abiotic $\mathrm{O}_{2}$ to build up in the atmosphere. This continues until $\mathrm{O}_{2}$, itself a noncondensable gas, overwhelms surface sinks to reach a sufficiently high atmospheric abundance that it can establish a cold trap, and halt the loss of water vapor. For an abiotic planet that is largely Earth-like except for the $\mathrm{N}_{2}$ inventory, Wordsworth and Pierrehumbert (2014) calculate that this proposed mechanism could result in planets with a very Earth-like $\mathrm{O}_{2}$ partial pressure $(\sim 0.15$ bars), while losing significant amounts of water.

\subsection{Enhanced $M d$ warf premain sequence stellar luminosity}

Another mechanism that may be capable of generating thousands of bars of abiotic $\mathrm{O}_{2}$ explores the impact of the premain sequence, super-luminous phase of young $\mathrm{M}$ dwarf stars on terrestrial planet environments (Luger and Barnes, 2015). The extended contraction phase of premain sequence young stars makes them significantly more luminous (e.g., Baraffe et al., 1998) than they will be when they enter the main sequence hydrogen burning phase. Consequently, planets that form in what will become the main sequence 
habitable zone are subjected early on to very high levels of radiation (Lissauer, 2007). This super-luminous phase is longer for lower mass $M$ dwarfs than other stellar spectral types, and can extend for up to 1 billion years (Gyr: Baraffe et al., 1998). Modeling suggests that this super-luminous phase can drive the loss of up to several Earth ocean equivalents of water for an $\mathrm{M}$ dwarf $\mathrm{HZ}$ terrestrial planet (Luger and Barnes, 2015; Tian, 2015). The resultant waterrich atmosphere will be susceptible to photolysis and hydrogen escape, and may produce hundreds or thousands of bars of $\mathrm{O}_{2}$ (Luger and Barnes, 2015; photolysis of an Earth ocean of water can produce $\sim 240$ bars of $\mathrm{O}_{2}$ on an Earthlike planet, Kasting, 1997).

The amount of $\mathrm{O}_{2}$ generated in this scenario is a strong function of stellar spectral type, XUV flux, original water inventory, planetary mass, and the position of the planet in the habitable zone. Planets in the outer regions of the habitable zone of M0-M3V stars are less likely to generate significant amounts of abiotic $\mathrm{O}_{2}$ (Luger and Barnes, 2015), as are planets orbiting M0-M3V dwarfs with lower stellar $\mathrm{XUV}$, as slower water loss may preclude $\mathrm{O}_{2}$ generation and buildup (Tian, 2015). However, for planets orbiting later type M dwarfs (M4V and later) depending on the planet's original water inventory, mass, stellar parameters, and the strength of surface sinks (Rosenqvist and Chassefière, 1995; Schaefer et al., 2016), up to several hundreds of bars of photolytically produced $\mathrm{O}_{2}$ could build up in the atmosphere (Luger and Barnes, 2015). For the recently discovered Proxima Centauri b, orbiting an M5.5V star, estimates of ocean loss during the $169 \pm 13$ Myr (Barnes et al., 2018) before the planet enters the habitable zone are sensitive to the assumed form of the XUV evolution. Results for global water loss range from less than one ocean $\left(<250\right.$ bars $\mathrm{O}_{2}$ generated), assuming a constant XUV flux for the host star for the first 3 Gyr (Ribas et al., 2016), up to 3-10 Earth ocean equivalents lost (750-2500 bars of $\mathrm{O}_{2}$ generated), assuming that the XUV scales with the decreasing bolometric luminosity (Barnes et al., 2018).

For terrestrial planets, if substantial $\mathrm{CO}_{2}$ is also outgassed over time, and the planet has little or no surface ocean in which to sequester $\mathrm{CO}_{2}$, then these types of worlds could sustain atmospheres with large quantities of both $\mathrm{CO}_{2}$ and $\mathrm{O}_{2}$ (Meadows et al., in press). Over time the $\mathrm{O}_{2}$ may be lost and $\mathrm{CO}_{2}$ may continue to accumulate. Although we have evidence that Venus lost a global "ocean" that was at least $3 \mathrm{~m}$ deep (de Bergh et al., 1991), any abiotic $\mathrm{O}_{2}$ generated was subsequently lost to surface or interior sinks (Rosenqvist and Chassefieré et al., 1995; Hamano et al., 2013; Schaefer et al., 2016) or top-of-atmosphere loss processes. The latter may include the recently discovered "electric wind" surrounding Venus, which can strip $\mathrm{O}_{2}$ ions from the planet (Collinson et al., 2016).

\subsection{Stellar spectrum driven photochemical production}

Abiotic $\mathrm{O}_{2}$ and its proxy, $\mathrm{O}_{3}$, can also be formed through planetary photochemistry of $\mathrm{CO}_{2}$. Each photochemical reaction requires photons that exceed a threshold energy level-or equivalently, photons at wavelengths shorter than a threshold wavelength-to be absorbed by and split the molecule undergoing photolysis. Planetary photochemistry is strongly sensitive to the UV spectral energy distribution of the parent star (e.g., Segura et al., 2003, 2005, 2010; Grenfell et al., 2007, 2014; Rugheimer et al., 2013, 2015), and in particular the ratio of shorter to longer wavelength UV radiation, which can split molecules and drive reactions that change the composition of the atmosphere, without relying on atmospheric escape.

The ultimate composition of the planetary atmosphere will then depend on the sources and sinks for photochemical reactions provided by the planetary environment. For example, photochemical abiotic $\mathrm{O}_{2}$ production will depend strongly on the source of $\mathrm{O}$ atoms, which is primarily controlled by the abundance and photolysis rates of atmospheric $\mathrm{CO}_{2}, \mathrm{SO}_{2}, \mathrm{H}_{2} \mathrm{O}$, and other O-bearing gases. The atmospheric sink will depend on the availability of $\mathrm{H}$ atoms in the atmosphere from $\mathrm{H}_{2}, \mathrm{H}_{2} \mathrm{~S}$, and hydrocarbons. Although water vapor can be sourced from a planetary ocean, the availability of other gases, such as $\mathrm{CO}_{2}, \mathrm{SO}_{2}$, and reducing (H-bearing) gases, is governed by the planet's volcanic outgassing rates, which can be sustainable on long geological timescales.

In particular, $\mathrm{CO}_{2}$ is likely a common atmospheric gas on terrestrial planets. In the Solar System, $\mathrm{CO}_{2}$ dominates the atmospheric composition of Venus and Mars, and was likely a significant component of the early Earth's atmosphere as well (Kasting et al., 1993; Sheldon et al., 2006; Sleep, 2010; Driese et al., 2011). The key reactions to produce $\mathrm{O}_{2}$ from $\mathrm{CO}_{2}$ photolysis include $\mathrm{CO}_{2}+\mathrm{h} v \rightarrow \mathrm{CO}+\mathrm{O}$, and the collisional recombination reaction $\mathrm{O}+\mathrm{O}+\mathrm{M} \rightarrow \mathrm{O}_{2}+\mathrm{M}$, where $\mathrm{M}$ is a third molecule that carries away excess energy to stabilize the collisional product. The yield of $\mathrm{O}_{2}$ from this process depends on how efficiently the back reaction to regenerate $\mathrm{CO}_{2}$ can occur. This, in turn, depends on the atmospheric abundance of catalysts such $\mathrm{a}_{\mathrm{x}}$ (e.g., from water vapor; Tian et al., 2014) or $\mathrm{NO}_{\mathrm{x}}$ (e.g., from cosmic rays; Grenfell et al., 2012) (c.f. Yung and DeMore, 1999; Stock, et al., 2017).

Aqueous reactions may also remove atmospheric $\mathrm{O}_{2}$, especially if the planet supports a surface ocean, and these reactions are also important for our understanding of abiotic $\mathrm{O}_{2}$ generation. For example, the rate of reaction of dissolved $\mathrm{CO}$ and $\mathrm{O}_{2}$ to reform $\mathrm{CO}_{2}$, and thereby draw down atmospheric $\mathrm{O}_{2}$, is poorly understood, but is crucial to understanding the final balance of $\mathrm{O}_{2}$ in the atmosphere (Harman et al., 2015). Similarly, weathering of surface crust (e.g., Anbar et al., 2007), and the sequestration of $\mathrm{O}_{2}$ into the planetary mantle (e.g., Hamano et al., 2013; Schaefer et al., 2016) are key processes that control $\mathrm{O}_{2}$ draw down, and could result in abiotic $\mathrm{O}_{2}$ buildup if slow. Conversely, if these processes are aggressive enough, they could produce a false negative for biologically produced $\mathrm{O}_{2}$ by scrubbing photosynthetically generated $\mathrm{O}_{2}$ from a planetary atmosphere, as likely happened over Earth's history, and discussed in Section 2.

Several groups have identified potential photochemical mechanisms to generate abiotic $\mathrm{O}_{2}$ and $\mathrm{O}_{3}$ on terrestrial planets in the habitable zone (Domagal-Goldman and Meadows, 2010; Hu et al., 2012; Domagal-Goldman et al., 2014; Tian et al., 2014; Gao et al., 2015; Harman et al., 2015). However, the amounts of abiotic $\mathrm{O}_{2}$ and $\mathrm{O}_{3}$ generated in these simulations differ, in part, due to different assumptions about the abundance of available catalysts to drive recombination of $\mathrm{CO}_{2}$, or destruction of $\mathrm{O}_{2}$ and $\mathrm{O}_{3}$, which can be affected by the availability of $\mathrm{H}_{2} \mathrm{O}$ and the spectrum of the star, as well as the efficiency of the $\mathrm{CO}$ and $\mathrm{O}_{2}$ reaction in seawater. The mechanism that could produce 
the largest signal from $\mathrm{CO}_{2}$ photolysis requires a desiccated, cold, H-poor atmosphere. This water-poor atmosphere cannot support photolytic generation of the $\mathrm{OH}$ catalyst that accelerates $\mathrm{CO}_{2}$ recombination (Gao et al., 2015). In this scenario, a catalytic cycle feedback with $\mathrm{O}_{3}$ formation combines with the suppression of $\mathrm{CO}_{2}$ recombination, and photochemical models predict stable atmospheric fractions of $\mathrm{O}_{2}$ near $15 \%$.

In another proposed mechanism, recombination of photolyzed $\mathrm{CO}_{2}$ can be slowed by a parent star (typically an $\mathrm{M}$ dwarf) with a higher far-ultraviolet (FUV; $0.122<\lambda<0.2 \mu \mathrm{m}$ ) to mid-ultraviolet (MUV; $0.2<\lambda<0.3 \mu \mathrm{m}$ ) and near-ultraviolet (NUV; $0.3<\lambda<0.44 \mu \mathrm{m}$ ) ratio when compared with the Sun. The higher FUV photolyzes $\mathrm{CO}_{2}$, but the lower MUV-NUV radiation inhibits the photolysis of water and other $\mathrm{HO}_{\mathrm{x}}$ chemistry that would drive recombination (Tian et al., 2014; Harman et al., 2015). For the cases considered for this mechanism, atmospheric $\mathrm{O}_{2}$ abundances as high as $0.2 \%$ to $6 \%$ are predicted, with higher values corresponding to little or no $\mathrm{O}_{2}$ sinks in the planetary environment (Harman et al., 2015). When more realistic sinks are included, abiotic $\mathrm{O}_{2}$ abundances are reduced by many orders of magnitude (e.g., Harman et al., 2015).

As another possible abiotic mechanism for $\mathrm{O}_{2}$ buildup, Léger et al. (2011) discussed the efficacy of known surface catalysts for abiotic photogeneration of $\mathrm{O}_{2}$ from water splitting, but concluded that it was highly unlikely that sufficient catalysis could occur on a habitable planet to generate a false positive. However, a subsequent study argued that this may be possible for a planet with shallow oceans, strong NUV, and significantly large areas of surface $\mathrm{TiO}$ to produce Earth-like quantities of $\mathrm{O}_{2}$ from the splitting of water $>1$ billion years (Narita et al., 2015).

$\mathrm{O}_{3}$ can often serve as a proxy for $\mathrm{O}_{2}$, especially in the MIR where $\mathrm{O}_{2}$ does not have spectral features (e.g., Des Marais et al., 2002). However, abiotic $\mathrm{O}_{3}$ could also be formed through photolysis of the abiotic $\mathrm{O}_{2}$ generated by ocean loss, with calculated values ranging from $1 \%$ of PALs of $\mathrm{O}_{3}$ up to values comparable with Earth's current $\mathrm{O}_{3}$ abundance for planets orbiting in the $\mathrm{HZ}$ of $\mathrm{M}$ dwarfs, depending on whether liquid water remains (Meadows et al., 2018). However, the spectral slope of the UV radiation of the star could also photochemically generate $\mathrm{O}_{3}$, even without buildup of $\mathrm{O}_{2}$. FUV radiation can favor the generation of $\mathrm{O}_{3}$ through photolysis of $\mathrm{CO}_{2}$ (and $\mathrm{O}_{2}$ ), whereas MUV or NUV radiation photolytically destroys $\mathrm{O}_{3}$. Consequently, abiotic $\mathrm{O}_{3}$ could accumulate - even without significant generation of $\mathrm{O}_{2}$-for stars with the highest FUV to MUV ratios. $\mathrm{O}_{3}$ was produced at $10 \%$ of Earth's current $\mathrm{O}_{3}$ column abundance in the simulations of Domagal-Goldman et al. (2014) for M dwarf planets, without appreciable buildup of abiotic $\mathrm{O}_{2}$.

Although the mechanisms that generate abiotic $\mathrm{O}_{2}$ and $\mathrm{O}_{3}$ are driven primarily by the interaction of the incident stellar spectrum with the planetary atmosphere, they can be balanced by the destruction or sequestration of $\mathrm{O}_{2}$ and $\mathrm{O}_{3}$ in the planetary environment. These losses could be through photolysis, catalysis, catalytic recombination into $\mathrm{CO}_{2}$, or interaction with the planetary surface and ocean, if present. The net atmospheric accumulation of $\mathrm{O}_{2}$ and $\mathrm{O}_{3}$ is highly sensitive to these boundary conditions, which include weathering rates and aqueous sinks for $\mathrm{CO}$, all of which are currently poorly understood.

\subsection{Summary of false positives}

Recent research indicates that there are several mechanisms that could produce abiotic $\mathrm{O}_{2}$ and $\mathrm{O}_{3}$ in a planet's atmosphere, with each presenting a potential false positive to different degrees. A summary of key components of this information is presented in Figure 2. Two of the mechanisms allow water to enter a planet's stratosphere where it is photolyzed, and the $\mathrm{H}$ atoms lost to space, resulting in $\mathrm{O}_{2}$ buildup in the planet's upper atmosphere. Water entering the stratosphere is either enabled by loss of an ocean in a runaway greenhouse process (Luger and Barnes, 2015) - a mechanism that is most effective for late-type (i.e., less massive) $\mathrm{M}$ dwarfs - or through lack of noncondensable gases in the planetary atmosphere, which could affect planets orbiting stars of any spectral type (Wordsworth and Pierrehumbert, 2014). The runaway mechanism could produce an $\mathrm{O}_{2}$-dominated atmosphere of hundreds of bars, and the lack of noncondensable gases could potentially result in atmospheres that are $\sim 15 \% \mathrm{O}_{2}$. Earth-like quantities of $\mathrm{O}_{2}$ have also been proposed to be generated by the splitting of liquid water by a surface $\mathrm{TiO}$ photocatalyst (Narita et al., 2015).

The other major class of processes that build up abiotic $\mathrm{O}_{2}$ relies on the photolysis of $\mathrm{CO}_{2}$ and circumstances that inhibit $\mathrm{CO}_{2}$ recombination from $\mathrm{CO}$ and $\mathrm{O}_{2}$ (Hu et al., 2012; Tian et al., 2014; Gao et al., 2015; Harman et al., 2015). For photochemical production without atmospheric escape, $\mathrm{O}_{2}$ abundances as high as $0.2 \%$ to $6 \%$ are predicted, with higher values corresponding to little or no $\mathrm{O}_{2}$ sinks in the planetary environment. More realistic modeling of sinks can reduce these estimates by orders of magnitude (e.g., DomagalGoldman et al., 2014; Harman et al., 2015). Finally $\mathrm{O}_{3}$ may be considered a proxy for $\mathrm{O}_{2}$ in a planetary atmosphere, and large abundances of abiotic $\mathrm{O}_{3}$ may build up in the massive $\mathrm{O}_{2}$-rich atmospheres possible after ocean loss (Meadows et al., 2018), although in these cases large amounts of $\mathrm{O}_{2}$ will also be present. Domagal-Goldman et al. (2014) were not able to generate large abundances of $\mathrm{O}_{2}$ from $\mathrm{CO}_{2}$ photolysis for habitable planets orbiting $\mathrm{M}$ dwarfs, but did produce potentially detectable $\mathrm{O}_{3}$ column abundance as high as $10 \%$ of Earth's modern abundance.

In most cases, however, the mechanism for abiotic production of $\mathrm{O}_{2}$ or $\mathrm{O}_{3}$ leaves a "tell," an impact on the planetary environment that may be detectable. These indications can range from the presence of collisionally induced absorption from $\mathrm{O}_{2}$ molecules that collide more frequently in dense, $\mathrm{O}_{2}$ rich postocean-loss atmospheres (Schwieterman et al., 2016; Meadows et al., 2018), $\mathrm{CO}$ from the photolysis of $\mathrm{CO}_{2}$ (Schwieterman et al., 2016), lack of water vapor (Gao et al., 2015), lack of collisionally induced absorption from $\mathrm{N}_{2}$ (Schwieterman et al., 2015b), to the absence of reducing gases (Domagal-Goldman et al., 2014). In the following section we describe the observations needed to search for $\mathrm{O}_{2}$ in a terrestrial planetary atmosphere and to discriminate whether that $\mathrm{O}_{2}$ is abiotic or biological in origin based on characteristics of the parent star and the planetary environment.

\section{Observational Requirements for Detecting and Discriminating $\mathrm{O}_{2}$ From Potential False Positives}

Exoplanet observing techniques required to characterize an exoplanet environment and search for life include time- and 


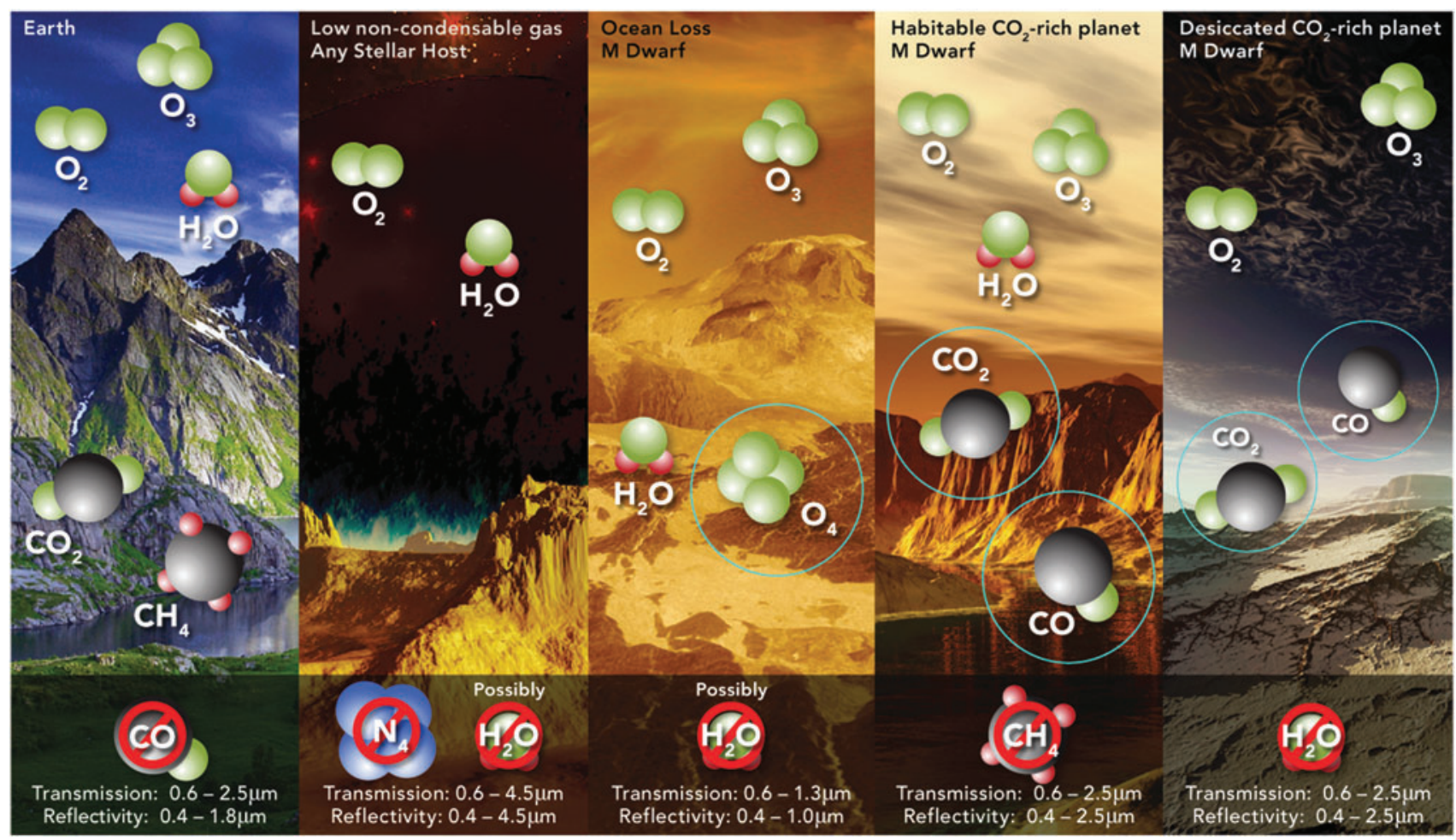

FIG. 2. Potential false positive mechanisms for $\mathrm{O}_{2}$. This cartoon summarizes the atmospheric mechanisms by which $\mathrm{O}_{2}$ could form abiotically at high abundance in a planetary atmosphere (Meadows, 2017). The extreme left panel is Earth, the four panels to the right show the different mechanisms and their observational discriminants. Circled molecules, if detected, would help reveal a false positive mechanism, a lack of detection of the "forbidden" molecules in the bottom shaded bar would also help to reveal the false positive mechanism. For example, the presence of $\mathrm{CO}$ and $\mathrm{CO}_{2}$, and the absence of $\mathrm{CH}_{4}$, is a strong indicator for a photochemical source of $\mathrm{O}_{2}$ from the photolysis of $\mathrm{CO}_{2}$ on a habitable $\mathrm{CO}_{2}$-rich $\mathrm{M}_{\text {dwarf }}$ planet (Figure credit: Ron Hasler).

wavelength-dependent photometry, phase-dependent photometry, and spectroscopy. Time-resolved information is valuable because it can show planetary rotation and changing cloud patterns (Cowan and Strait, 2013), and measurements taken over the course of a planetary orbit may reveal seasonality. Seasonal changes may be biologically driven (e.g., Keeling et al., 1976) and so may be valuable as a separate metric from the presence of abundant $\mathrm{O}_{2}$ for detecting life on an exoplanet. Phase-dependent observations, that is, those taken when the exoplanet is at different points in its orbit and may be seen at different illumination phases, can reveal interesting features such as ocean glint or the presence of forward-scattering clouds, which may be water (Robinson et al., 2010). At thermal wavelengths, phase-dependent observations can be used to search for planetary day-night temperature differences and atmospheric molecular absorption (Meadows et al., 2018).

Spectra, meanwhile, open the door to detailed characterization and quantification of surface features and atmospheric gas abundances. Atmospheric gas abundances derived from spectra would then be used as inputs to atmospheric photochemical-climate models to predict the gas surface fluxes required to sustain the observed abundance; quantifying these surface fluxes may aid in distinguishing true biosignatures from abiotic processes (DomagalGoldman et al., 2011). Three main observational techniques will be used to obtain spectra of exoplanets in the next two decades-transmission spectroscopy, high-contrast direct imaging spectroscopy, and high-resolution spectroscopy. In this section, we discuss the pros and cons of different exoplanet observing techniques, factors affecting target selection considerations, and which measurements will be the most valuable for characterizing terrestrial exoplanet environments and searching for life.

Transmission spectroscopy with the JWST, scheduled for launch in early 2019, will likely be our first opportunity to search for $\mathrm{O}_{2}$ in the atmosphere of one to three habitable zone Earth-sized planets (Cowan et al., 2015). Transmission spectroscopy, where a spectrum of the planetary atmosphere is taken when a planet transits in front of and is "backlit" by its parent star, offers advantages and disadvantages for exoplanet characterization. This technique is most likely to succeed for habitable zone planets orbiting M dwarfs, where a stronger signal from the planet can be obtained because a terrestrial-sized planet can block out a more significant fraction of the small $\mathrm{M}$ dwarf's light, and the proximity of the planet to the star results in many more transits in a given time span. The longer path length through the backlit planetary atmosphere enhances the impact of higher altitude aerosols and trace gases on the transmission spectrum (Fortney, 2005) when compared with the downward-looking observations of reflected light from the planet expected from direct imaging. Because the atmosphere is "backlit" by the star, the decrease in signal-to-noise $(\mathrm{S} / \mathrm{N})$ ratio with increasing wavelength is less problematic than in reflected 
light observations, where the planet becomes less reflective at longer wavelengths.

However, a number of processes can limit the ability of transmission observations to probe a planet's troposphere. These processes include refraction (Bétrémieux and Kaltenegger, 2013, 2014; Misra et al., 2014b), condensates/ aerosols (Kreidberg et al., 2014; Misra and Meadows, 2014; Morley et al., 2015), and the optical depth contributed by atmospheric constituents (Meadows et al., 2018). Transmission cannot observe the planetary surface, and the accessible regions of the atmosphere of a habitable planet will likely be water-poor layers above the water-preserving cold trap, potentially making it more difficult to detect atmospheric water vapor by using this technique (Meadows et al., 2018). However, several $\mathrm{O}_{2}, \mathrm{O}_{3}$, and $\mathrm{O}_{4}$ bands may be accessible.

Direct imaging requires telescopes with sufficient spatial resolution, and instrumentation that can suppress the star's brightness, so that the much fainter planet can be imaged. This allows the planet to be studied through either photometry or spectroscopy. Angular/spatial resolution for a telescope scales with $1 / \mathrm{D}$, where $\mathrm{D}$ is the diameter of the telescope. Larger telescopes provide smaller spatial resolution, and so can separate planets that are closer to their star. High-performing technologies to suppress light from the central star include internal occulters to block or redirect the star's light after it enters the telescope (coronagraphs; Stapelfeldt et al., 2015), or external occulter spacecraft that fly between the telescope and the star (starshades; Seager et al., 2015). Direct imaging can potentially probe the entire atmospheric column and even retrieve information on the planetary surface for clear sky scenes. This technique is, therefore, less sensitive to aerosols, and more sensitive to the deeper, near-surface atmosphere than transmission spectroscopy (Arney et al., 2016, 2017).

The first space-based direct imaging mission to be launched will be the Wide Field Infrared Survey Telescope (WFIRST) in the mid-2020s, but this coronagraph-enabled $2.4 \mathrm{~m}$ telescope is unlikely to be able to detect and characterize terrestrial planets (Robinson et al., 2016). However, the 2020 Astronomy Decadal Survey will review the potential for WFIRST to have a "rendezvous" with an external occulter (Seager et al., 2015), which may provide the starlight suppression suitable for discovering rocky worlds.

Development is currently underway for more capable direct imaging mission concepts such as the Habitable Exoplanet Imaging Mission (HabEx) and the Large UltraViolet Optical Infrared Surveyor (LUVOIR), one of which could be launched in the mid-2030s. Such a mission could enable observations of smaller Earth-sized targets, and search for $\mathrm{O}_{2}$ and other atmospheric constituents in their spectra (Postman et al., 2010; Bolcar et al., 2015; Dalcanton et al., 2015; Rauscher et al., 2015; Seager et al., 2015; Stapelfeldt et al., 2015; Mennesson et al., 2016).

Complementary to these space-based telescopes, future 30-40 m ground-based observatories (extremely large telescopes, ELTs; e.g., Kasper et al., 2008), coming on line in the mid-late 2020s, will also offer the capability to detect and measure the spectra of perhaps 10 or more terrestrial exoplanets in the habitable zones of $\mathbf{M}$ dwarfs (Crossfield, 2016), depending on the occurrence rate of $\mathrm{M}$ dwarf $\mathrm{HZ}$ planets (e.g., Dressing and Charbonneau, 2015). Transmission spectroscopy of later-type $\mathrm{M}$ dwarf exoplanets using high-spectral resolution spectrographs could obtain some of the earliest ground-based ELT measurements (Szentgyorgyi et al., 2016), and search for $\mathrm{O}_{2}$ in exoplanet atmospheres (Rodler and López-Morales, 2014). By combining coronagraphs and adaptive optics with high-resolution spectrographs (e.g., Lovis et al., 2017), and employing template-matching techniques to disentangle exoplanet spectral lines from Earth's own atmospheric absorption features (e.g., Snellen et al., 2015), spectra of nontransiting exoplanets may also be possible. Ground-based direct imaging observations at 3-10 $\mu \mathrm{m}$ for planets orbiting in the habitable zones of F, G, and K stars (Brandl et al., 2014; Snellen et al., 2013; Quanz et al., 2015; Crossfield, 2016) are also anticipated for the late 2020s.

Direct imaging observations are constrained by inner- and outer-working angles (IWAs and OWAs) - the smallest and largest planet-star separations over which the observatory is capable of achieving adequate starlight suppression to reveal the planet. For coronagraphs, these angles scale with $\lambda / D$, the telescope's resolution element, where $\lambda$ is the wavelength of the observations and $D$ is the telescope mirror diameter. Desirable IWAs on coronagraphs are typically 2-3 $\lambda / D$ and largely determined by the effectiveness and stability of the coronagraph working in conjunction with its telescope and spacecraft; the OWA is determined by the format size of the coronagraph's deformable mirrors. For starshades, the IWA depends on the geometric ratio between the size of the starshade and the separation distance between the starshade and its telescope. Because its IWA is independent of the telescope aperture, the starshade can allow observations of close-in planets (improved IWA) with relatively smaller telescope apertures. However, the telescope must still be at least large enough to have the requisite spatial resolution to separate the planet and the star, and to collect sufficient light from the planet in a reasonable amount of exposure time.

The IWA will tend to make observations of planets in the habitable zone of $\mathrm{M}$ dwarfs difficult, because these stars are intrinsically faint, and the habitable zone is 20 times closer to the star than it would be for a G dwarf. For instance, the Earthsized planet orbiting in the habitable zone of the M dwarf star Proxima Centauri is only 0.0485 AU away from its parent star (Anglada-Escudé et al., 2016), which at the Sun-Proxima Centauri distance corresponds to a planet-star angular separation on the sky of 37 milliarcseconds at maximum elongation. Therefore, although direct imaging of Earthlike planets orbiting the closest $\mathrm{M}$ dwarfs may be possible for larger observatories like the 16-m diameter LUVOIR concept, and LUVOIR may have as many as $8 \%$ of its accessible targets orbiting M dwarfs, direct imaging space missions tend to focus primarily on planets orbiting $\mathrm{F}, \mathrm{G}$, and $\mathrm{K}$ stars for which the planet-star angular separations are larger.

\subsection{Target selection}

Careful target selection may help reduce the likelihood of false positive $\mathrm{O}_{2}$, although the stellar and planetary properties most likely to result in false positive $\mathrm{O}_{2}$ are still being explored. Several of the proposed false positive mechanisms for $\mathrm{O}_{2}$ production are more likely to occur for planets around later type $\mathrm{M}$ dwarfs, which may be more susceptible to early ocean loss and buildup of $\mathrm{O}_{2}$ from subsequent $\mathrm{H}$ loss (Baraffe et al., 1998; Luger and Barnes, 2015). In 
contrast, planets in the habitable zones of $\mathrm{F}, \mathrm{G}$, and $\mathrm{K}$ stars will not experience a super-luminous host star for a significant period of time. M dwarfs may also have NUV/FUV flux ratios that are more favorable for photochemical production of $\mathrm{O}_{2}$ or $\mathrm{O}_{3}$ (Domagal-Goldman et al., 2014; Tian et al., 2014; Harman et al., 2015). Systems older than a few Gyr may be preferred, to allow time for biologically generated $\mathrm{O}_{2}$ to overwhelm sinks and rise in the atmosphere, and to increase the probability that abiotic $\mathrm{O}_{2}$ generated during the star's super-luminous premain sequence phase has been depleted through sequestration in the planetary environment (Schaefer et al., 2016) or lost to space (Collinson et al., 2016; Ribas et al., 2016; Airapetian et al., 2017; Dong et al., 2017; Garcia-Sage et al., 2017).

When searching for biosignatures, it would, therefore, be advantageous to select older F, G, K, or earlier type M dwarf targets (M0-M3), where the habitable zones are likely safer from a false positive for $\mathrm{O}_{2}$ through premain sequence ocean loss (Luger and Barnes, 2015). It will also be important to have measurements, or proxies, to estimate the UV spectrum of the M dwarf host star. However, some of these false positive mechanisms are less dependent on the host star, and are instead tied to planetary properties such as a lack of noncondensable gas species in the planetary atmosphere (Wordsworth and Pierrehumbert, 2014) or an abundance of surface TiO (Narita et al., 2015). In these cases, abiotic $\mathrm{O}_{2}$ production could occur for planets with suitable characteristics orbiting stars of any spectral type. Target selection for detailed follow-up will instead be informed by characterization of the planet's environment, and specifically a census of bulk gases such as $\mathrm{N}_{2}, \mathrm{O}_{2}$, and $\mathrm{CO}_{2}$. More theoretical work is needed to better understand the factors that affect habitability so that the planets most likely to be able to support life are chosen for detailed follow-up.

We are now entering a new era wherein the first observations to constrain the nature of terrestrial exoplanet atmospheres are being taken (e.g., de Wit et al., 2016), potentially providing some of the first empirical information relevant to habitability and false positive processes. Early observations of exoplanets with high levels of incident starlight such as GJ1132b (Berta-Thompson et al., 2015) and the inner TRAPPIST-1 planets (Gillon et al., 2016, 2017) may reveal key characteristics of their planetary environments that shape abiotic $\mathrm{O}_{2}$ generation and persistence, and even provide an observational test for the limits of the habitable zone.

\subsection{Key measurements of environmental characteristics that provide context for $\mathrm{O}_{2}$ detection}

Should a promising candidate planet be identified, there are several key observations that can help place detection of atmospheric $\mathrm{O}_{2}$ into context, and help discriminate between biological and abiological sources. The first of these is detailed knowledge of the parent star, including its spectral type, metallicity, age, observed or inferred UV to infrared spectrum, and a measure of its activity levels, including the amplitude and frequency of stellar flares. The spectral type and age help to identify the likely stellar evolutionary path of the star (Baraffe et al., 1998), which drives planetary evolution, including atmospheric loss processes, which may produce false positives. The spectrum of the star is needed as input to atmospheric climate and photochemistry models, which will be used to determine atmospheric temperature, and the efficiency of photochemical production and destruction of $\mathrm{O}_{2}$ and $\mathrm{O}_{3}$.

Basic properties of the planet are also important, such as radius, orbit, and mass. Radius is most readily determined for transiting exoplanets where the fractional dimming of the star during the transit is a direct consequence of the planet's size, or more explicitly, its cross-sectional area. However, only a small fraction of habitable zone planets will transit (from $0.5 \%$ to $1.5 \%$ for $\mathrm{G}$ to $\mathrm{M}$ dwarf host stars). For nontransiting planets, determining a planetary radius is more challenging, as a size-albedo degeneracy exists in reflected light, as a planet could have a given measured brightness either because it is small but reflective, or because it is larger and less reflective. The range of plausible albedos for a terrestrial planet could easily span 0.06 to 0.9 , potentially generating a factor of 7 uncertainty in the estimated size of the planet. Observations at MIR wavelengths-wherein emissivities for most terrestrial planetary surfaces are close to $1-$ may be used to infer planetary radius, especially if a color temperature can be derived from the shape of the MIR spectrum. JWST MIR thermal observations of Proxima Centauri $b$ at different points in its orbit (a thermal phase curve) may reveal daynight temperature differences (Kreidberg and Loeb, 2016; Turbet et al., 2016; Meadows et al., 2018), whereas future ELTs may have the ability to image terrestrial planets in the habitable zones of a small sample of $M$ dwarfs at wavelengths near $10 \mu \mathrm{m}$ (e.g., Quanz et al., 2015). If the planet can be directly imaged at multiple epochs in its orbit, then the relative astrometry of the star and planet can be used to derive the orbital parameters. This can be done in either reflected light or in thermal emission, but the better angular resolution available at shorter wavelengths favors the use of reflected light imaging.

If the planet is not directly imaged, then its orbit may be determined indirectly-by measuring its dynamical influence on the host star or other planets in the system. The planetary mass and orbit, including its inclination, can be determined with sufficiently precise stellar astrometry to measure the reflex motion of the star induced by the planet. Stellar radial velocity (RV) measurements can determine all the orbital elements except for inclination, the angle of orientation of the planet's orbital plane to the observer. Since RV measures only the radial component of the star's orbital velocity, without knowing the orbital plane orientation, these measurements provide only a lower limit on the planetary mass (e.g., Anglada-Escudé et al., 2016).

For nontransiting planets, this RV orbital inclination ambiguity can be resolved by combining RV with imaging detections at more than one epoch and over a significant fraction of the planet's orbit, or through observations of emitted or reflected light from the planet in high-resolution spectroscopy. With the latter technique, the spectral shift in planetary atmospheric atomic or molecular lines reveals the RV of the planet itself (e.g., Snellen et al., 2015), and since the planetary orbital velocity is known from the stellar RV period, the inclination can be derived. Terrestrial planets will be extremely challenging to observe this way, and may have to await second- or third-generation instruments on ground-based ELTs. However, searching for emission from visible light aurorae on planets orbiting $M$ dwarfs may 
help improve the contrast for planetary RV measurements (Luger et al., 2017). In the special case of transiting planets in multiplanet systems, the perturbations of one planet by another can sometimes be measured as changes in the timing of successive transits (transit timing variations) and allow the planetary masses to be derived (e.g., Gillon et al., 2017).

Another key planetary characteristic needed to interpret an observation of $\mathrm{O}_{2}$ in a planetary atmosphere is the presence of water, which could be in the form of atmospheric gas, condensed clouds, and/or a surface ocean. Although the possibility that the planet can support liquid water is more likely for an Earth-like planet within the surface liquid water habitable zone (Kasting et al., 1993; Kopparapu et al., 2013), it is not guaranteed, as water may not have been delivered to the planet during its formation (Lissauer, 2007; Raymond et al., 2007), or it may have been subsequently lost (Luger and Barnes, 2015; Airapetian et al., 2016; Ribas et al., 2016; Barnes et al., 2018).

If water is present, it can help rule out several of the false positive mechanisms that rely on water loss (Luger and Barnes, 2015) or photochemistry in an extremely water-poor atmosphere (Gao et al., 2015). Knowledge of the water vapor profile-and the detection of condensate clouds that form at the water vapor cold trap-would rule out another false positive mechanism associated with high-altitude photolysis in atmospheres without a cold trap (Wordsworth and Pierrehumbert, 2014). However, if residual water persists after water loss from either early ocean loss or lack of noncondensable gases, then abiotic $\mathrm{O}_{2}$ and water vapor may both be seen in the spectrum (Meadows et al., 2018). Absorption features for water occur throughout the visible $(0.65,0.7$, $0.73,0.8$, and $0.95 \mu \mathrm{m}), \operatorname{NIR}(1.1,1.4$, and 1.8-2.0 $\mu \mathrm{m})$, and

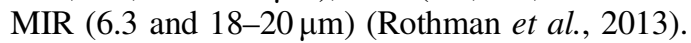

Observing liquid surface water itself may be challenging and will likely only be possible for reflected light observations of nearby planets with an optimal orbital plane orientation. Observing liquid surface water is a less ambiguous habitability indicator than observations of gaseous water spectral features, which could be present in uninhabitable steam atmospheres. One such method to detect liquid surface water is ocean glint, caused by specular reflection off a smooth surface (Cox and Munk, 1954). Glint has been used to detect the presence of hydrocarbon seas on the surface of Saturn's moon Titan (Stephan et al., 2010) and water oceans in spacecraft views of Earth (Robinson et al., 2014).

Previously, Robinson et al. (2010, 2014) used a sophisticated 3D spectral Earth model to show that the spectral behavior of Earth deviates strongly from Lambertian (i.e., isotropic) scattering behavior toward crescent phases when specular reflection would be most apparent. Forward scattering from glint may mimic forward scattering behavior of clouds, but Robinson et al. (2010) showed that ocean glint even in the presence of realistic clouds (covering $\sim 50 \%$ of the planet) is up to a factor of 2 brighter than forward scattering from clouds and a nonglinting (i.e., Lambertian) ocean. Glint is most readily observed at phase angles within about $60^{\circ}$ of new phase and for orbital plane inclinations that are no more than $30^{\circ}$ from edge on to the observer. Glint from Earth's surface is most detectable at wavelengths near $0.8-0.9 \mu \mathrm{m}$ where Earth's atmosphere is relatively transparent (although it may also be observed in the continuum between the longer $1.1,1.4$, and $1.9 \mu \mathrm{m}$ water vapor bands).
Terrestrial exoplanet glint measurements are not possible in transmission, and outside the realm of feasibility for JWST phase curve measurements, even for the exoplanet orbiting the closest star (Proxima Centauri b; Anglada-Escudé et al., 2016). In the case of Proxima, measurement precision for detecting glint in an NIR phase curve would need to be smaller than $10^{-8}$ (Meadows et al., 2018), but this is significantly smaller than typical JWST noise floor estimates, which are on the order of $10^{-5}$ (Greene et al., 2016), and so unachievable. However, these measurements may be achievable for future ELTs and for nearby habitable zone targets observed by large space-based exoplanet characterization missions such as LUVOIR (Meadows et al., 2018).

Tectonic activity is critical to the long-term habitability of planets, as it both recycles elements required for life (e.g., Berner, 2004; Wordsworth, 2016) and serves as a global thermostat that regulates climate over geological timescales (Walker et al., 1981). Direct observations of tectonic activity will be extremely challenging, but observable impacts of tectonics on the planetary environment may be more accessible. A heterogeneous distribution of surfaces seen in maps generated by using time-resolved photometry (Cowan et al., 2009) may suggest the presence of continents. Gases released from volcanic eruptions could also be sought (Kaltenegger and Sasselov, 2009; Kaltenegger et al., 2010), but the resulting formation and accumulation of aerosols in the upper atmosphere would likely provide larger, timevariable signals (Hu et al., 2013; Misra et al., 2015). The "ingredients" for tectonic activity could also be inferred based on the elemental composition of the system. Planetary mass and radius, informed by the $\mathrm{Fe}, \mathrm{Si}$, and $\mathrm{Mg}$ abundance of the host star, could be used to constrain the interior composition and structure and the likelihood that a planet is tectonically active (Young et al., 2014; Dorn et al., 2015).

Additional contextual information to guide interpretation of a planet's habitability can come from atmospheric pressure. Rayleigh scattering could be used to indicate pressure above the visible surface (Benneke and Seager, 2012; von Paris et al., 2013), whether that be the planetary surface or a cloud or haze layer, as is the case for Venus. However, interpretation of these measurements can be compromised by a surface that strongly absorbs or scatters in the blue, as is the case for Mars and Earth, respectively. Rayleigh scattering will be less useful for planets orbiting M dwarfs, as the host star produces little radiation at the blue end of the visible spectrum wherein Rayleigh scattering dominates, significantly lowering the $\mathrm{S} / \mathrm{N}$ at these wavelengths (Meadows et al., 2018).

Collision-induced features that are more sharply dependent on atmospheric pressure may provide an even more sensitive measurement of atmospheric pressure. For example, $\mathrm{N}_{4}$ or $\mathrm{O}_{4}$ features $\left(\mathrm{N}_{2}-\mathrm{N}_{2}\right.$ or $\left.\mathrm{O}_{2}-\mathrm{O}_{2}\right)$ seen in the NIR could be used to indicate higher pressures (Misra et al., 2014a; Schwieterman et al., 2015b). $\mathrm{N}_{4}$ absorbs strongly near $4.15 \mu \mathrm{m}$, just shortward of the strong $4.3 \mu \mathrm{m} \mathrm{CO}_{2}$ band, and creates a strong spectral effect for abundances of $p \mathrm{~N}_{2}>$ 0.5 bar (Schwieterman et al., 2015b). The location of this strongly diagnostic feature in the infrared creates challenges for direct-imaging missions currently under study due to IWA and thermal background limitations to much shorter wavelengths. However, it remains perhaps the only feature capable of directly confirming large quantities of $\mathrm{N}_{2}$ in an atmosphere (Schwieterman et al., 2015b). 
Finally, a census of atmospheric gases should be performed to inform planetary composition, chemistry, and climate. The composition of the atmosphere will be important for assessing the potential of the planet to generate photochemical false positives and for calculating surface fluxes required to maintain $\mathrm{O}_{2}$ and $\mathrm{O}_{3}$ in the planetary atmosphere. It will also help identify potential sources of nutrients and energy for life at the surface. The atmospheric composition, and in particular knowledge of greenhouse gas abundances and distributions, when combined with information on the incoming stellar flux, can also be used to model the climate of the planet and its potential surface habitability.

However, to determine the planet's atmospheric composition, we will need not only good observations, but also robust techniques for spectroscopic retrieval that can maximize information content from low spatial- and spectral-resolution data of poor sensitivity (e.g., Irwin et al., 2008; Madhusudhan and Seager, 2009; Benneke and Seager, 2012; Line et al., 2014; Waldmann et al., 2015). The observation type (e.g., transmission, emission, and direct), wavelength coverage, and spectral resolution (among other factors) affect how complete a spectral search for atmospheric gases will be.

Degeneracies between physical parameters can also complicate inferences of the atmospheric composition. Such degeneracies have been described between the following: gas mixing ratios and temperature in secondary eclipse (Waldmann et al., 2015); mixing ratios, planetary radius, and reference pressure in transit (Benneke and Seager, 2012; Heng and Kitzmann, 2017); mixing ratios and cloud coverage in transit (Barstow et al., 2013); spectrally inactive gases (Benneke and Seager, 2012); and patchy clouds and mean molecular weight in transit (Line and Parmentier, 2016) to name a few. These degeneracies tend to arise as a result of low $\mathrm{S} / \mathrm{N}$ observations that cover a narrow wavelength range. Consequently, many degeneracies can be lifted by using multiple instruments that together cover a wide wavelength (von Paris et al., 2013b; Barstow et al., 2015).

However, more work is needed to assess biases that arise due to the choice of parameterization in simple forward models (c.f. Feng et al., 2016), as well as how additional information on the star, planet, and entire system of interest may help to enable a more complete understanding of the atmospheric composition in the event of continued exoplanet spectrum limitations.

\subsection{Detecting the $\mathrm{O}_{2}$ signal from a photosynthetic biosphere}

On our own Earth, $\mathrm{O}_{2}$ is abundant and evenly mixed throughout the atmosphere. The principal means of detecting $\mathrm{O}_{2}$ is through its spectral features in the visible and NIR spectra at 0.69 (B-band), 0.76 (A-band), and $1.27 \mu \mathrm{m}$. On Earth-like exoplanets, it may be accessible to both transmission spectroscopy, which will likely probe above most of the deep atmosphere, and direct imaging, which can potentially sample the atmosphere all the way to the planetary surface. A spectral resolution of 70 would be needed to resolve the A-band at Earth-like abundances (Robinson et al., 2016). The $\mathrm{O}_{4}$ collisionally induced absorption features in the visible and NIR spectra may also be used as proxies for $\mathrm{O}_{2}$, and may help constrain its concentration (Misra et al., 2014a). They are weakly present in Earth's direct imaging spectrum (Tinetti et al., 2006) but become much stronger when seen in transmission (Pallé et al., 2009) or in denser $\mathrm{O}_{2}$ atmospheres (Misra et al., 2014a). $\mathrm{O}_{2}$ is accessible to planned transmission spectroscopy and direct imaging at 0.76 and $1.27 \mu \mathrm{m}$, if the telescope and star will support it. But the $1.27 \mu \mathrm{m} \mathrm{O}_{2} / \mathrm{O}_{4}$ band may produce a stronger signal for planets orbiting late-type $M$ dwarfs that have low output at wavelengths shortward of $0.8 \mu \mathrm{m}$, and so induce low planetary transmission or reflected light signals. Ground-based high-resolution spectroscopy may be able to detect $\mathrm{O}_{2}$ on some habitable zone terrestrial planets orbiting $\mathrm{M}$ dwarfs by using the B- and A-bands in the visible spectrum (Snellen et al., 2015; Lovis et al., 2017).

Although obtaining well-constrained abundances for these $\mathrm{O}_{2}$-bearing molecules may be challenging with firstgeneration observatories, observations of even the presence or absence of different bands of these molecules may help constrain $\mathrm{O}_{2}$ abundances. The strong dependence of $\mathrm{O}_{3}$ and $\mathrm{O}_{4}$ features on $\mathrm{O}_{2}$ concentrations could also be highly diagnostic of both chemistry and pressure. This combination of features can allow, for example, discrimination between atmospheres that contain $1 \% \mathrm{O}_{2}, 20 \% \mathrm{O}_{2}$, and $90 \% \mathrm{O}_{2}$ (Section 4.4).

$\mathrm{O}_{3}$ features in the UV could also point to the presence of $\mathrm{O}_{2}$ in atmospheres with lower levels of $\mathrm{O}_{2}$, when $\mathrm{O}_{2}$ itself is not detectable. Figure 3 shows Proterozoic Earth with 0.01 bar of $\mathrm{CO}_{2}, 0.0003$ bar of $\mathrm{CH}_{4}$ and $0.1 \%$ the PAL of $\mathrm{O}_{2}$, as suggested by recent studies of chromium isotopes in geological samples from the mid-Proterozoic $(\sim 1.8-0.8 \mathrm{Ga}$; Planavsky et al., 2014b). $\mathrm{O}_{2}$ itself is extremely weak in the spectrum, and is not discernable in this plot, but $\mathrm{O}_{3}$ produces a relatively strong UV (Hartley) band from 0.2 to $0.3 \mu \mathrm{m}$ that is not saturated, unlike the present Earth's, and so there is still a relatively high signal in the bottom of the band. Consequently, obtaining an accurate measurement of the flux level at the bottom of the band, needed to quantify abundance, is potentially easier than for current Earth-like conditions.

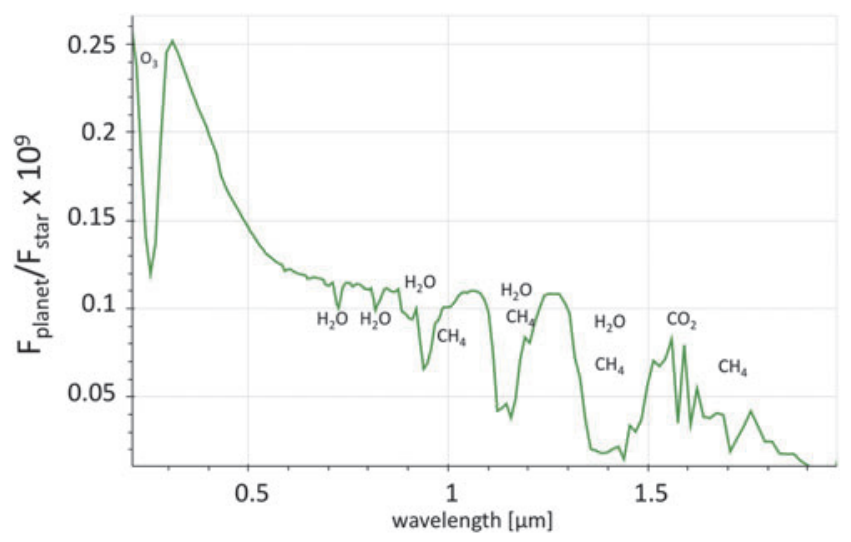

FIG. 3. Reflectance spectrum of the Proterozoic Earth assuming $0.1 \% \mathrm{PAL} \mathrm{O}_{2}$ (Planavsky et al., 2014b), generated using the Atmos coupled climate-photochemical model (Arney et al., 2016). Note the absence of strong $\mathrm{O}_{2}$ features, but its photochemical byproduct $\mathrm{O}_{3}$ produces a relatively strong feature in the UV from 0.2 to $0.3 \mu \mathrm{m}$. This figure was generated using the LUVOIR simulator available at https:// asd.gsfc.nasa.gov/luvoir and described in Robinson et al., (2016). LUVOIR, Large UltraViolet Optical Infrared Surveyor. (Credit: G. Arney) 
Searching for $\mathrm{O}_{2}$ in the atmosphere of Proxima Centauri b with ground-based telescopes may be possible in the next 5 years. Perhaps the most rapid path to this goal was suggested by Lovis et al. (2017), who proposed upgrades and the development of a coupling interface between the SPHERE high-contrast imager and the new ESPRESSO high-resolution spectrograph on the $8.2 \mathrm{~m}$ very large telescope. The combined high-contrast/high-resolution technique would provide the desired angular separation and contrast sensitivity of $10^{-7}$ in reflected light. This would make it possible to probe the $\mathrm{O}_{2}$ bands at $0.63,0.69$, and $0.76 \mu \mathrm{m}$, along with water vapor at $0.72 \mu \mathrm{m}$ and $\mathrm{CH}_{4}$ at $0.75 \mu \mathrm{m}$. Lovis et al. (2017) calculated that a $\sim 4$-sigma detection of $\mathrm{O}_{2}$ on an Earth-like Proxima Cen b could be made in about 60 nights of telescope time, spread over 3 years to observe the planet at maximum separation from the star. Even better results for the $\mathrm{M}$ dwarf habitable zone planets within $8 \mathrm{pc}$ are likely possible with the ELTs slated for first light in the mid-to-late 2020s (e.g., Rodler and López-Morales, 2014).

Future large space telescopes are being designed to detect $\mathrm{O}_{2}$ for exoplanets orbiting $\mathrm{F}, \mathrm{G}$, and $\mathrm{K}$ stars. The design requirements for the HabEx and LUVOIR observatory concepts include the ability to detect $\mathrm{O}_{2}$ in the atmospheres of neighboring Earth-like exoplanets (e.g., Dalcanton et al., 2015; Mennesson et al., 2016). Whether a planet's $\mathrm{O}_{2}$ spectral features are in practice detectable with these observatories will depend on several parameters, including the planet's $\mathrm{O}_{2}$ abundance and the distance of the planet-star system to Earth. For example, an Earth-like exoplanet orbiting a Sun-like star at $20 \mathrm{pc}$ would require integration times exceeding hundreds of hours to detect the $\mathrm{O}_{2} \mathrm{~A}$-band with sufficient $\mathrm{S} / \mathrm{N}$ for even a $6.5 \mathrm{~m}$ (JWST-sized) optical telescope with $2 \lambda / D$ IWA coronagraphic capability. For planet-star systems farther than about $20 \mathrm{pc}$, this important spectral feature would be unobservable. A larger telescope $(12.7 \mathrm{~m})$ could detect $\mathrm{O}_{2}$ out to about $40 \mathrm{pc}$ given the same IWA-although, again, requiring very long integration times. Figure 4 shows the integration time as a function of wavelength centered on the $\mathrm{O}_{2}$ A-band required for a $15 \mathrm{~m}$ LUVOIR-class telescope to obtain $\mathrm{S} / N=10$ for a planet identical to modern Earth orbiting a solar twin at a distance of $10 \mathrm{pc}$. The spectral resolution assumed here is 150 . For these assumptions, $10 \mathrm{~s}$ of hours are required to obtain S/ $N=10$ across most of the wavelength ranges shown. Figure 5 shows the spectrum for the planet that could be obtained across the UV-VIS-NIR wavelength range in $30 \mathrm{~h}$ per coronagraphic bandpass for this same observatory. The significant increase in the size of the error bars at longer wavelengths is due to thermal emission from the telescope, which is assumed to be heated to $270 \mathrm{~K}$. The spectral resolutions assumed for the UV-VIS-NIR channels for both Figures 4 and 5 are $R=20$ for the UV $(\lambda<0.4 \mu \mathrm{m}), R=150$ for the visible $(0.4<\lambda<0.85)$, and $R=100$ in the NIR $(\lambda>$ $0.85)$. Both were simulated by using a coronagraph noise model based on the one described in Robinson et al. (2016).

\subsection{Discriminating false positives}

Once $\mathrm{O}_{2}$ is detected in a planetary spectrum, searching for additional observed environmental features to place the $\mathrm{O}_{2}$ in context will aid in discriminating false positive $\mathrm{O}_{2}$ from

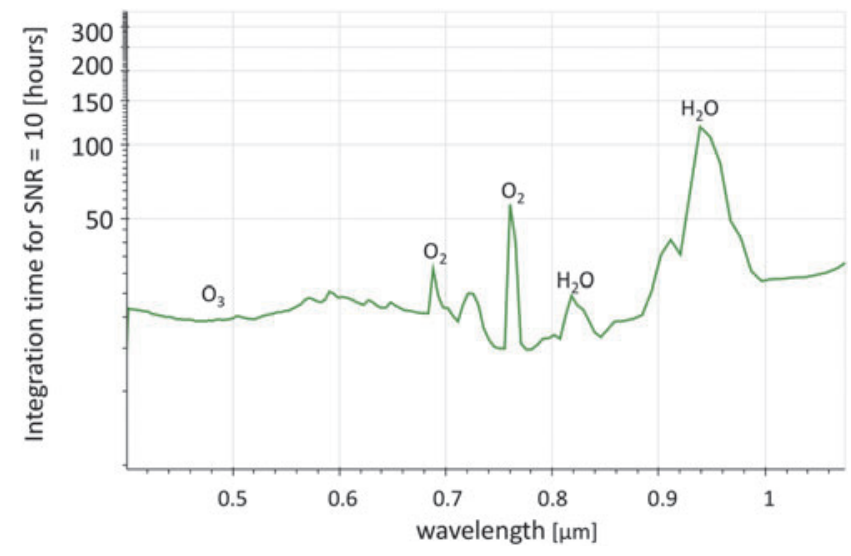

FIG. 4. The integration time as a function of wavelength required to obtain an $\mathrm{S} / \mathrm{N}=10$ for the modern Earth orbiting a star at $10 \mathrm{pc}$ for a $15 \mathrm{~m}$ LUVOIR-class telescope. The spectrum is roughly centered on the $\mathrm{O}_{2} 0.76 \mu \mathrm{m}$ A-band. Spectral resolution $=150$ in the visible region. $\mathrm{S} / \mathrm{N}=10$ can be obtained in $10 \mathrm{~s}$ of hours at most wavelengths. This figure was generated using the LUVOIR simulator available at https://asd.gsfc.nasa.gov/luvoir and described in Robinson et al. (2016) (credit: G. Arney).

true biological $\mathrm{O}_{2}$. Hereunder we describe currently known methods for observational discriminants for abiotic and biological $\mathrm{O}_{2}$.

4.4.1. Identifying $\mathrm{O}_{2}$ buildup from ocean loss. A proposed discriminant of the massive $\mathrm{O}_{2}$-rich atmosphere produced by the loss of oceans of water during the premain sequence phase of the host star is the appearance of $\mathrm{O}_{4}$ collisional complexes in the planet's spectrum (Schwieterman et al., 2016); these features are caused by collisioninduced $\mathrm{O}_{2}-\mathrm{O}_{2}$ absorption and are highly sensitive to atmospheric density. $\mathrm{O}_{4}$ features occur at $0.345,0.36,0.38$, $0.445,0.475,0.53,0.57$, and $0.63 \mu \mathrm{m}$ in the visible and at 1.06 and $1.27 \mu \mathrm{m}$ in the NIR (Greenblatt et al., 1990; Hermans et al., 1999; Maté et al., 1999; Richard et al., 2012; Schwieterman et al., 2016). These strong $\mathrm{O}_{4}$ features would indicate a $\mathrm{O}_{2}$ atmosphere that is likely too massive to be biologically produced (Schwieterman et al., 2016). In transmission observations, the NIR $\mathrm{O}_{4}$ bands at 1.06 and $1.27 \mu \mathrm{m}$ would be the best diagnostic spectral indicators of very high $\mathrm{O}_{2}$ buildup $\left(p \mathrm{O}_{2}>1\right.$ bar; Misra et al., 2014a; Schwieterman et al., 2016). Figure 6 shows a simulated transmission spectrum of a high $\mathrm{O}_{2}$ atmosphere with prominent $\mathrm{O}_{4}$ bands. These longer wavelength bands are not as badly affected by the increase in Rayleigh scattering as their counterparts at shorter wavelengths.

Using the photon-limited JWST instrument model of Deming et al. (2009), Schwieterman et al. (2016) found that JWST/NIRISS could detect the 1.06 and $1.27 \mu \mathrm{m}$ bands with an $\mathrm{S} / \mathrm{N}$ of $\sim 3$, assuming 65-h integrations (10 transits) of an Earth-like planet around a star like GJ 876 (M4V; $R=0.39$ $\mathrm{R}_{\odot}$ ) (Fig. 7). However, other, more conservative estimates suggest that no features in any high-molecular weight atmosphere could be detected by JWST due to a systematic noise floor (e.g., Greene et al., 2016). For direct imaging observations, the shorter wavelength $\mathrm{O}_{4}$ bands in the visible spectrum are now prominent against the Rayleigh scattering 


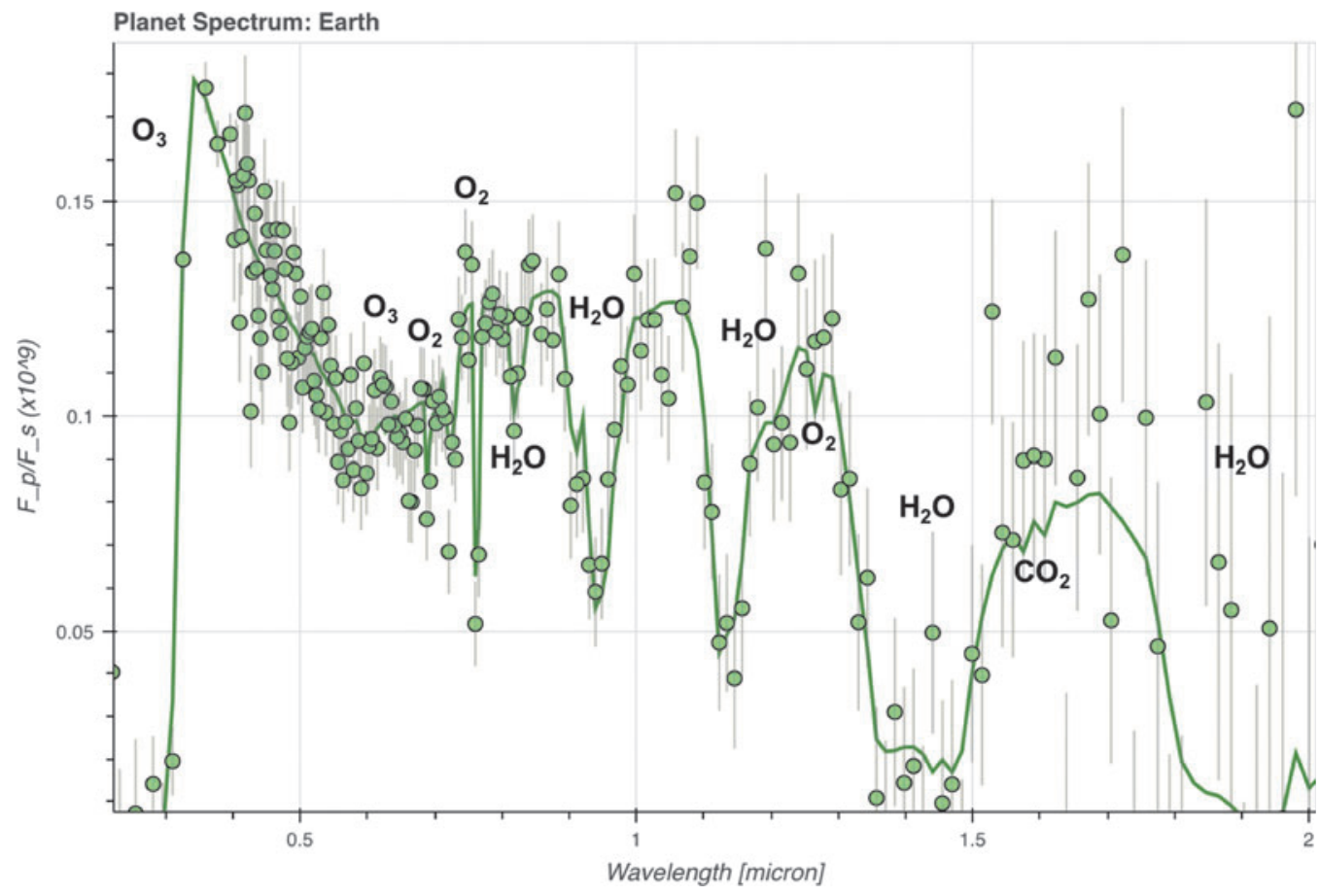

FIG. 5. The reflectance spectrum obtainable in $30 \mathrm{~h}$ by a $15 \mathrm{~m}$ space-based telescope observing modern Earth orbiting a Sun-like star at $10 \mathrm{pc}$. The gray bars denote the noise level, which increases significantly at wavelengths longward of $1.8 \mu \mathrm{m}$ due to thermal radiation from the telescope, which is assumed to be heated to $270 \mathrm{~K}$. This figure was generated using the LUVOIR simulator available at https://asd.gsfc.nasa.gov/luvoir and described in Robinson et al. (2016) (credit: G. Arney).

slope (Fig. 8). These bands can be obtained even within the shorter wavelength range constrained by the IWA for more distant targets, or for planets that are closer to their star as is the case for $\mathrm{M}$ and $\mathrm{K}$ habitable zone planets.

4.4.2. Identifying abiotic $\mathrm{O}_{2} / \mathrm{O}_{3}$ from $\mathrm{CO}_{2}$ photolysis. An atmosphere with abiotic $\mathrm{O}_{2}$ produced by $\mathrm{CO}_{2}$ photolysis is more likely to have three additional features as follows: (1) a sufficient UV flux from the host star, (2) high $\mathrm{CO}_{2}$ abundances in the atmosphere, and (3) photochemically produced $\mathrm{CO}$ (Hu et al., 2012; Domagal-Goldman et al., 2014; Tian et al., 2014; Harman et al., 2015; Gao et al., 2015). A caveat to this scenario is the possible presence of catalysts that could accelerate the destruction of abiotically generated $\mathrm{O}_{2}$ - producing lower and potentially undetectable abiotic $\mathrm{O}_{2}$ concentrations (Harman et al., 2017). However, if these catalysts are not present, strong $\mathrm{CO}$ features in the presence of abundant $\mathrm{O}_{2}$ and $\mathrm{CO}_{2}$ could signal a false positive scenario (Schwieterman et al., 2016; Meadows, 2017; bottom panels of Figs. 6 and 8). $\mathrm{CO}_{2}$ exhibits strong spectral features near $1.65,2$, and $4.3 \mu \mathrm{m}$, and weaker bands occur near $0.78,0.87,1.05$, and $1.2 \mu \mathrm{m}$ (Rothman et al., 2013). CO absorbs strongly near 2.35 and $4.6 \mu \mathrm{m}$. In practice, for exoplanet direct imaging space observatories that are not cryogenically cooled to lower than room temperature, simulations show that wavelengths longer than about $1.8 \mu \mathrm{m}$ may be effectively unobservable, except for the brightest targets, due to the overwhelming thermal background of the telescope itself (Fig. 5). In this case, only the weaker $1.65 \mu \mathrm{m} \mathrm{CO} \mathrm{CO}_{2}$ band and none of the $\mathrm{CO}$ bands would be available. In addition, the NIR spectrograph channel may not extend to long enough wavelengths to access these features. This would make it challenging to identify this false positive in direct imaging. However, these longer wavelengths may be accessible to JWST. Assuming the most productive scenario known for abiotic $\mathrm{O}_{2}$ generation from $\mathrm{CO}_{2}$ photolysis ( $p \mathrm{O}_{2}=0.06$ bars) for an Earth-like M-dwarf $\mathrm{HZ}$ planet with a prebiotic $\mathrm{N}_{2}-\mathrm{CO}_{2}-\mathrm{H}_{2} \mathrm{O}$ atmosphere (Harman et al., 2015), $\mathrm{CO}$ and $\mathrm{CO}_{2}$ bands shortward of $4.6 \mu \mathrm{m}$ could be detected with an $\mathrm{S} / \mathrm{N} \sim 3$. This assumes a 65-h (10 transit) observation by JWST NIRISS $(0.6-2.9 \mu \mathrm{m})$ and NIRSPEC $(2.9-5.0 \mu \mathrm{m})$ and photon-limited noise (Fig. 9; Schwieterman et al., 2016). As mentioned, the $\mathrm{CO}_{2}$ and $\mathrm{CO}$ bands are the strongest features in the simulated spectrum. $\mathrm{CO}_{2}$ may also be accessible at $15 \mu \mathrm{m}$ by using thermal phase curve measurements of 10 to $100 \mathrm{~s}$ of hours on JWST, even for nontransiting planets, provided there is adequate day-night temperature difference (Meadows et al., 2018).

4.4.3. Constraining abiotic $\mathrm{O}_{2}$ from low $\mathrm{N}_{2}$ inventories. The abiotic $\mathrm{O}_{2}$ mechanism posited by Wordsworth and Pierrehumbert (2014) suggests that low noncondensing gas inventories on Earth-like planets could lead to abiotic $\mathrm{O}_{2}$ buildup by lifting the tropospheric cold trap, allowing water to reach the stratosphere where it could be photolyzed. Atomic hydrogen would escape, leaving atomic oxygen behind to potentially build up in the atmosphere as $\mathrm{O}_{2}$. However, this scenario could be ruled out if the noncondensing gas abundance could be directly constrained. Throughout Earth's long 4.5-billion-year history, the dominant noncondensable gas has been $\mathrm{N}_{2}$. The $\mathrm{N}_{4}\left(\mathrm{~N}_{2}-\mathrm{N}_{2}\right.$; collision-induced absorption) feature overlaps with the $4.3 \mu \mathrm{m} \mathrm{CO}_{2}$ band (Fig. 10), but absorbs over a much broader 
FIG. 6. Transit transmission spectra of potential planetary environments with different $\mathrm{O}_{2}$ abundances for planet orbiting the M5.5V star Proxima Centauri (Meadows et al., 2018). Illustrating spectral features that can help distinguish photosynthetic from abiotically generated $\mathrm{O}_{2}$ in a planetary atmosphere. From top to bottom: selfconsistent Earth-like atmosphere with $50 \%$ cloud cover $\left(21 \% \mathrm{O}_{2}\right) ; 10$ bar abiotic $\mathrm{O}_{2}$ $\left(\begin{array}{ll}95 \% & \mathrm{O}_{2}\end{array}\right)$ atmosphere produced by early ocean loss with ocean remaining (purple) and desiccated (orange); 1 bar desiccated $\mathrm{CO}_{2} / \mathrm{CO} / \mathrm{O}_{2}$ atmosphere that has reached a kinetic-photochemical equilibrium between the photolysis rate of $\mathrm{CO}_{2}$ and kineticslimited recombination $\left(\begin{array}{lll}15 \% & \mathrm{O}_{2}\end{array}\right)$. Effective atmospheric radius in kilometers is on the left $y$ axes and transit depth is shown on the right $y$ axes. The photosynthetic source for $\mathrm{O}_{2}$ in the Earth-like case is made more likely by the presence of $\mathrm{O}_{2} / \mathrm{O}_{3}$, water, and methane. High $\mathrm{O}_{2}$ cases with and without water are distinguished by the presence of $\mathrm{O}_{4}$, and the behavior of the $0.5-0.7 \mu \mathrm{m}$ Chappuis band that is sensitive to tropospheric $\mathrm{O}_{3}$, which is more abundant in the desiccated case. The desiccated chemical equilibrium atmosphere is easily distinguished by its high levels of $\mathrm{CO}$.
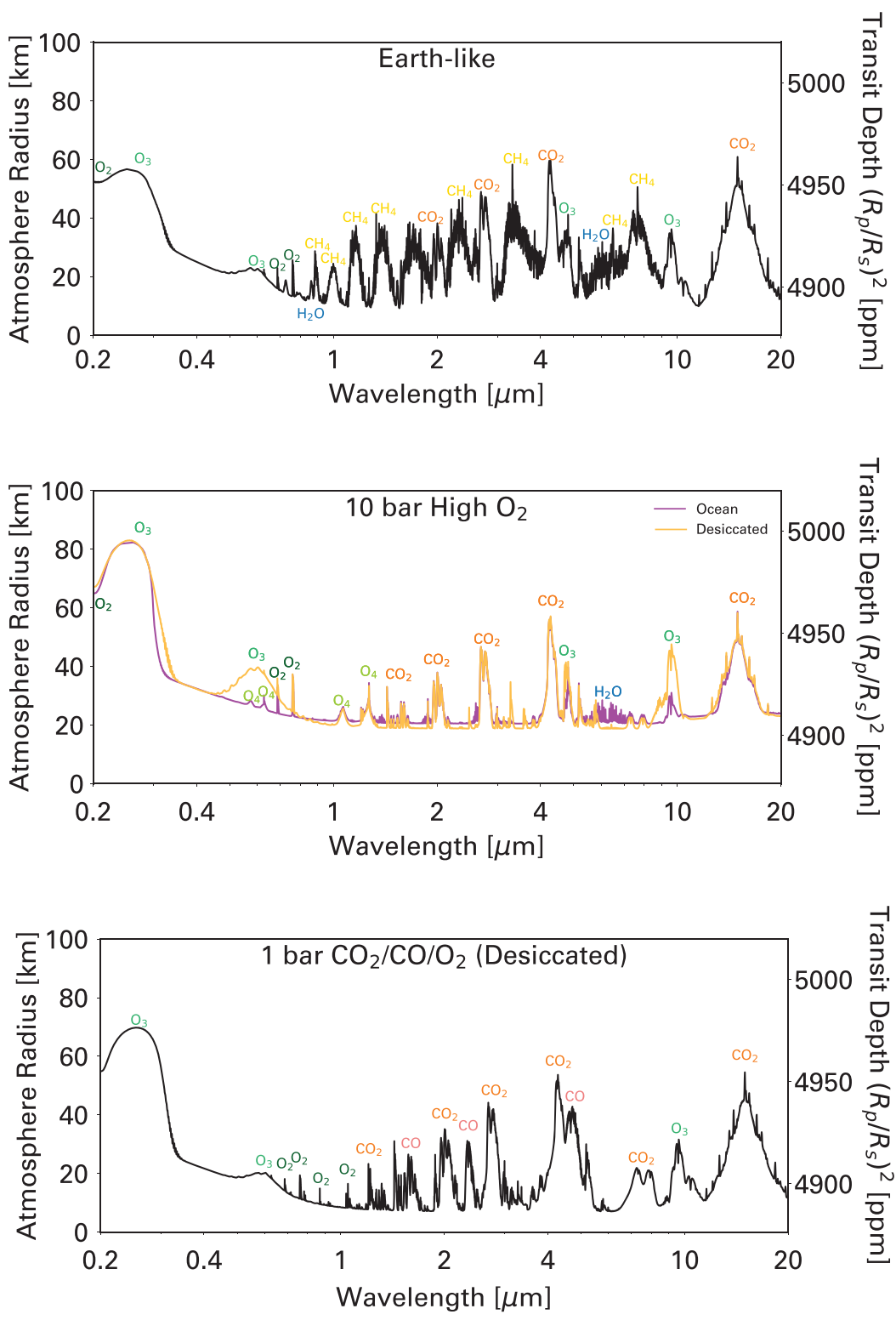
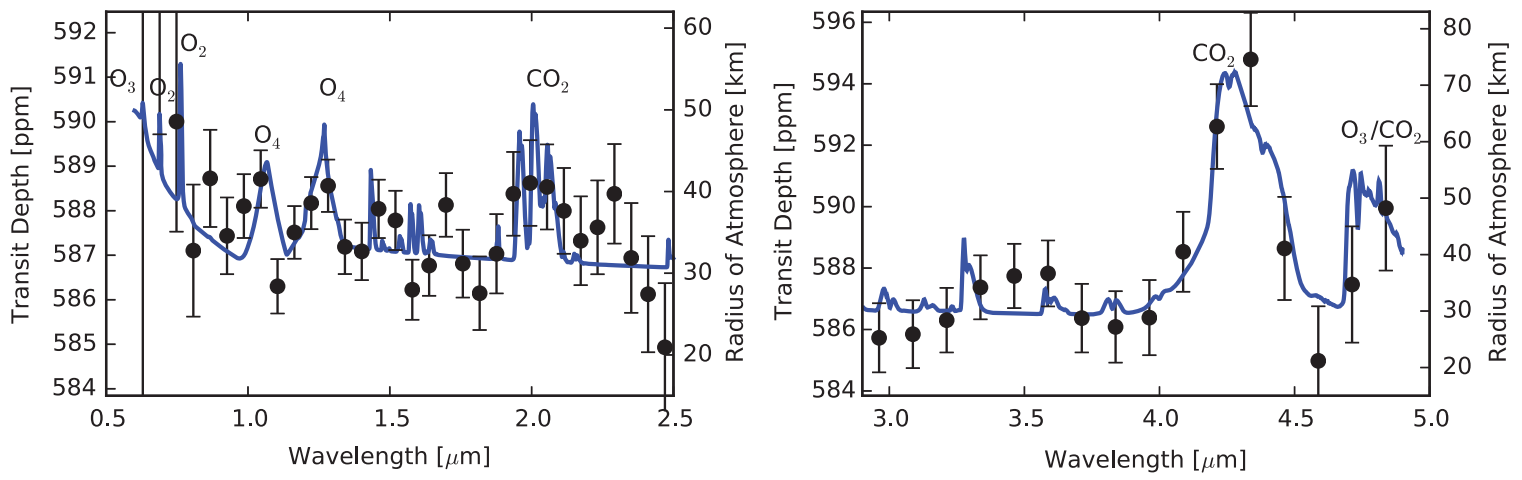

FIG. 7. Synthetic transmission spectrum of high $\mathrm{O}_{2}$ atmosphere with NIR $\mathrm{O}_{4}$ features at 1.06 and $1.27 \mu \mathrm{m}$. The model atmosphere is a hypothetical 100 bar $\mathrm{O}_{2}$ atmosphere left behind by massive $\mathrm{H}$ escape during premain sequence evolution (Luger and Barnes, 2015). Data and error bars $(1 \sigma)$ for simulated JWST-NIRISS (left) and JWST-NIRSpec (right) were calculated with the noise model of Deming et al. (2009) assuming $65 \mathrm{~h}$ integrations (10 transits of an Earth-size planet around GJ876) and photon-limited noise. Figure adapted from Schwieterman et al. (2016). JWST, James Webb Space Telescope; NIR, near-infrared. 

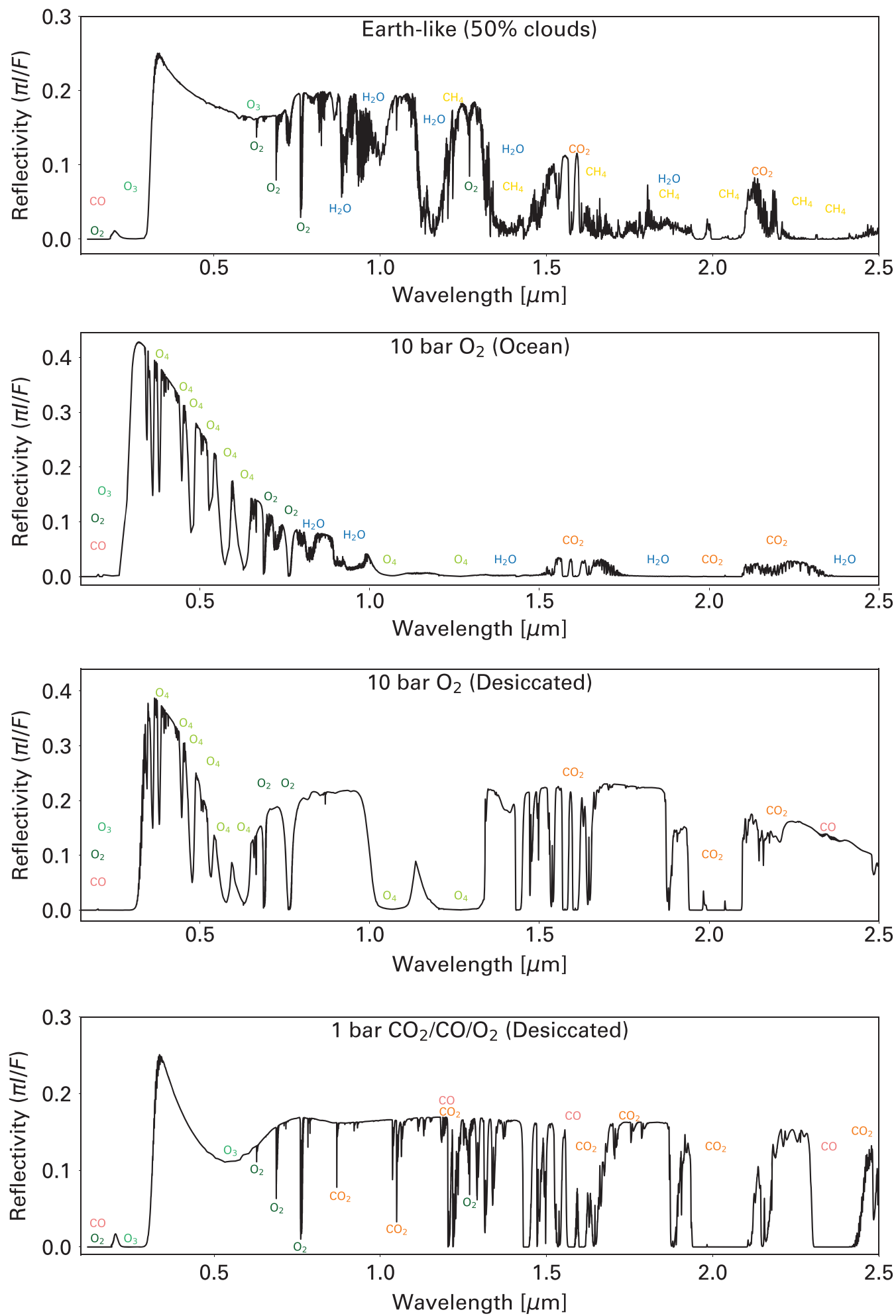

FIG. 8. Reflected light spectra of potential Proxima Centauri b climates from top to bottom: self-consistent Earth-like atmosphere with $50 \%$ cloud cover, 10 bar abiotic $\mathrm{O}_{2}$ atmosphere with ocean, 10 bar desiccated $\mathrm{O}_{2}$ atmosphere, 1 bar desiccated $\mathrm{CO}_{2} / \mathrm{CO} / \mathrm{O}_{2}$ atmosphere in kinetic-photochemical equilibrium (see Meadows et al., 2018). The $\mathrm{O}_{2}$ content in these atmospheres is produced from very different mechanisms: Earth $\mathrm{O}_{2}$ is biological, the 10 bar abiotic $\mathrm{O}_{2}$ atmospheres result from the super-luminous premain sequence evolution of the planet's $\mathrm{M}$ dwarf host star, and the last atmosphere is a 1 bar $\mathrm{CO}_{2}$ atmosphere that has $<1 \mathrm{ppm}$ hydrogen, resulting in a slow $\mathrm{CO}_{2}$ recombination timescale compared with its photolysis rate and Earth-like levels of $\mathrm{O}_{2}$. However, because hydrogen is also required to destroy ozone $\left(\mathrm{O}_{3}\right)$, this atmosphere exhibits more $\mathrm{O}_{3}$ and a stronger Chappuis $\mathrm{O}_{3}$ band at $\sim 0.6 \mu \mathrm{m}$. The 10 bar $\mathrm{O}_{2}$ atmospheres are easily distinguished from Earth-like atmospheres by their deep, wide $\mathrm{O}_{2}-\mathrm{O}_{2}\left(\mathrm{O}_{4}\right)$ collision-induced absorption bands. Moist versus desiccated abiotic $\mathrm{O}_{2}$ cases are distinguished primarily by the presence or absence of water features. Around $\mathrm{M}$ dwarf stars, Earth-like planets with biological and geophysical sources of methane result in longer atmospheric lifetimes and correspondingly deep, observable methane features (Segura et al., 2005; Rugheimer et al., 2015). 

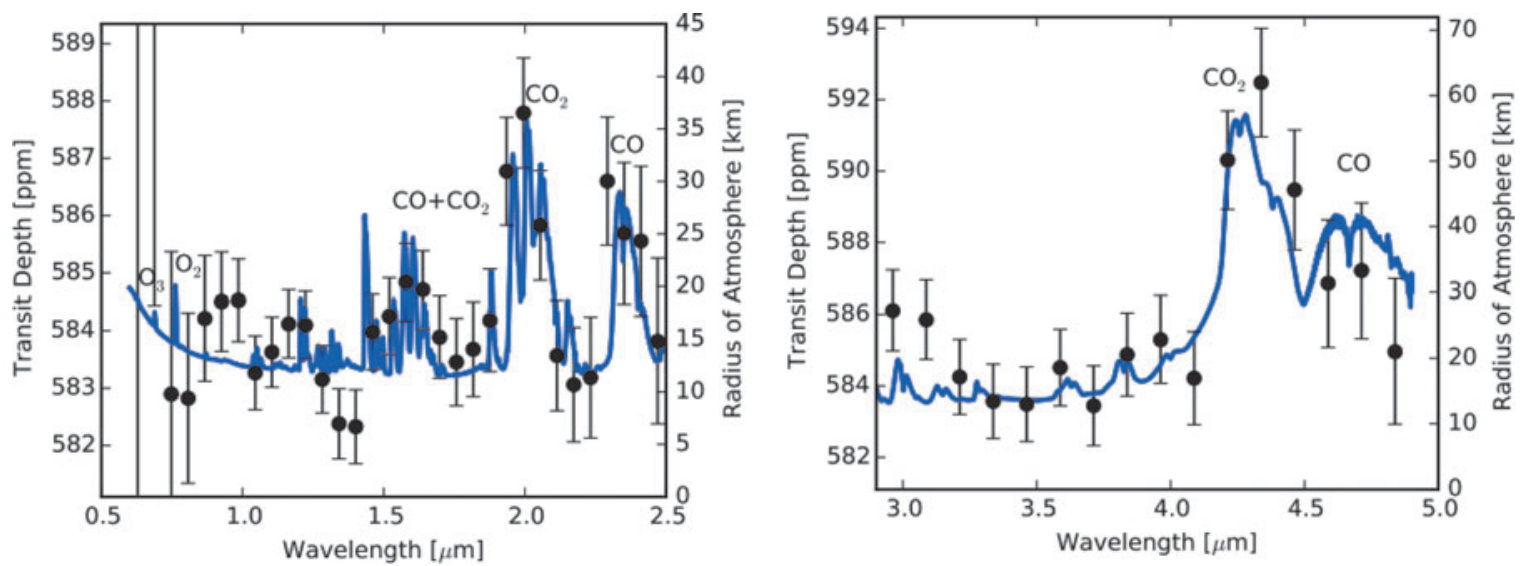

FIG. 9. Synthetic transmission spectrum with photochemical $\mathrm{O}_{2}$ and $\mathrm{CO}$ features. Model prebiotic atmosphere in photochemical equilibrium around GJ876 from Harman et al. (2015) containing $\sim 6 \%$ abiotic $\mathrm{O}_{2}$ and $\sim 1 \% \mathrm{CO}$. Data and error bars $(1 \sigma)$ for simulated JWST-NIRISS (left) and JWST-NIRSpec (right) were calculated with the noise model of Deming et al. (2009), assuming $65 \mathrm{~h}$ integrations (10 transits of an Earth-size planet around GJ876) and photon-limited noise. Figure adapted from Schwieterman et al. (2016).

region (Lafferty et al., 1996; Schwieterman et al., 2015b). In reflected light spectra, the influence of $\mathrm{N}_{4}$ on the $4.1 \mu \mathrm{m}$ region is strongly dependent on $\mathrm{N}_{2}$ abundance beyond $P_{0}=0.5$ bars (see Fig. 9; Schwieterman et al., 2015b). There is also a much weaker harmonic $\mathrm{N}_{4}$ band at $2.1 \mu \mathrm{m}$ (Shapiro and Gush, 1966), seen in the reflectance spectra of nitrogen ices on outer solar system bodies (Grundy and Fink, 1991), but this band is currently poorly characterized and probably too weak to create a measurable impact on plausible planetary atmospheres.

The spectral transmission depths due to $\mathrm{N}_{4}$ will vary, depending on the abundance of $\mathrm{CO}_{2}$ and the overall mean molecular weight of the atmosphere, but it can reach $10 \mathrm{ppm}$ for an $\mathrm{N}_{2}$-dominated atmosphere for an Earth-size planet transiting an M5V $\left(R=0.2 \mathrm{R}_{\odot}\right)$ star. Larger transmission depths may be possible for atmospheres with a significant low-molecular weight component such as $\mathrm{H}_{2}$ (Schwieterman et al., 2015b).

\subsection{Considerations for super-Earths}

The majority of the mentioned discussion assumed that the habitable planet being observed was close to Earth size, but terrestrial planets may have radii up to 1.5 times that of Earth (Weiss and Marcy, 2014; Rogers, 2015; Fulton et al., 2017). These super-Earth atmospheres may be less Earthlike, as the larger masses of terrestrial super-Earths increase their ability to retain lighter molecules, such as $\mathrm{H}_{2}$, and/or to produce substantial outgassing of heavier gases such as $\mathrm{H}_{2} \mathrm{O}$ and $\mathrm{CO}_{2}$. However, if their atmospheres are more Earth-like, the increased radius and gravity of larger planets will affect the detectability of $\mathrm{O}_{2}$ (c.f. Rauer et al., 2011) and false

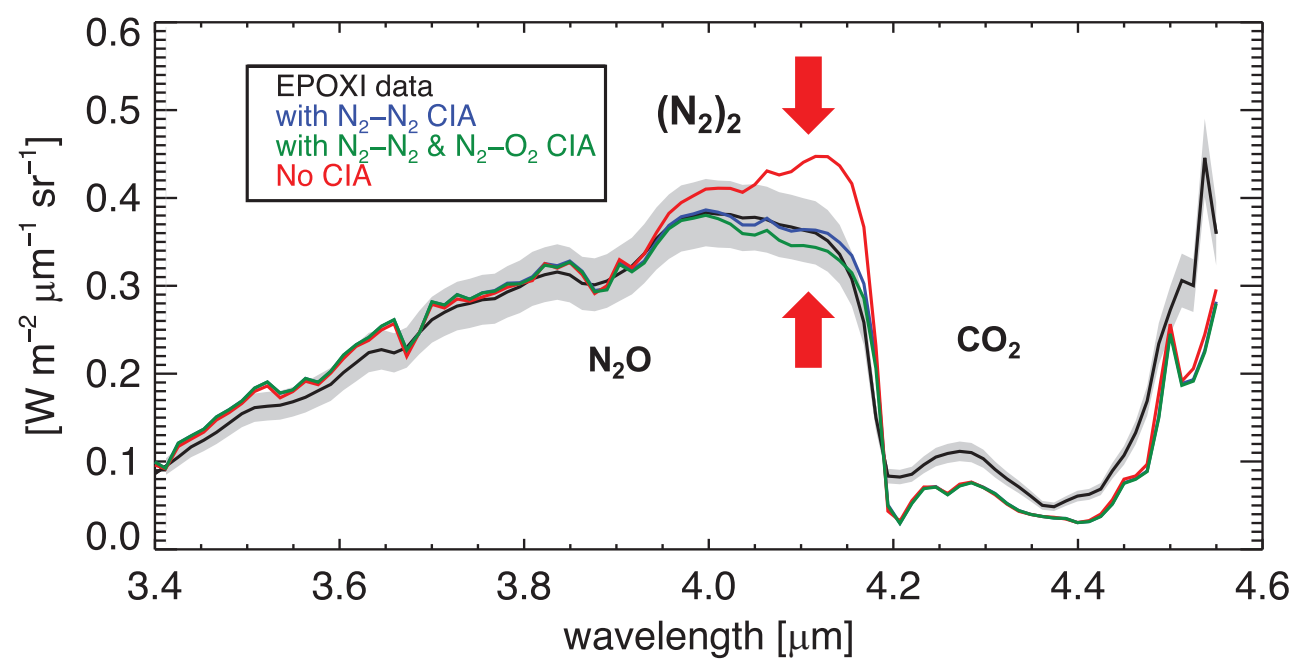

FIG. 10. The impact of $\mathrm{N}_{4}$ absorption in Earth's disk-averaged NIR spectrum. This plot shows the spectral differences between the cases with $\mathrm{N}_{4}$ absorption (blue and green) and the case without it (red), when compared with the EPOXI observations of the Earth in this wavelength range (black). $\mathrm{N}_{4}$ is clearly required to match the observed spectrum of the Earth near $4.1 \mu \mathrm{m}$. The gray band shows the calibration uncertainty for the EPOXI data (Klassen et al., 2008). Figure adapted from Schwieterman et al. (2015b). 


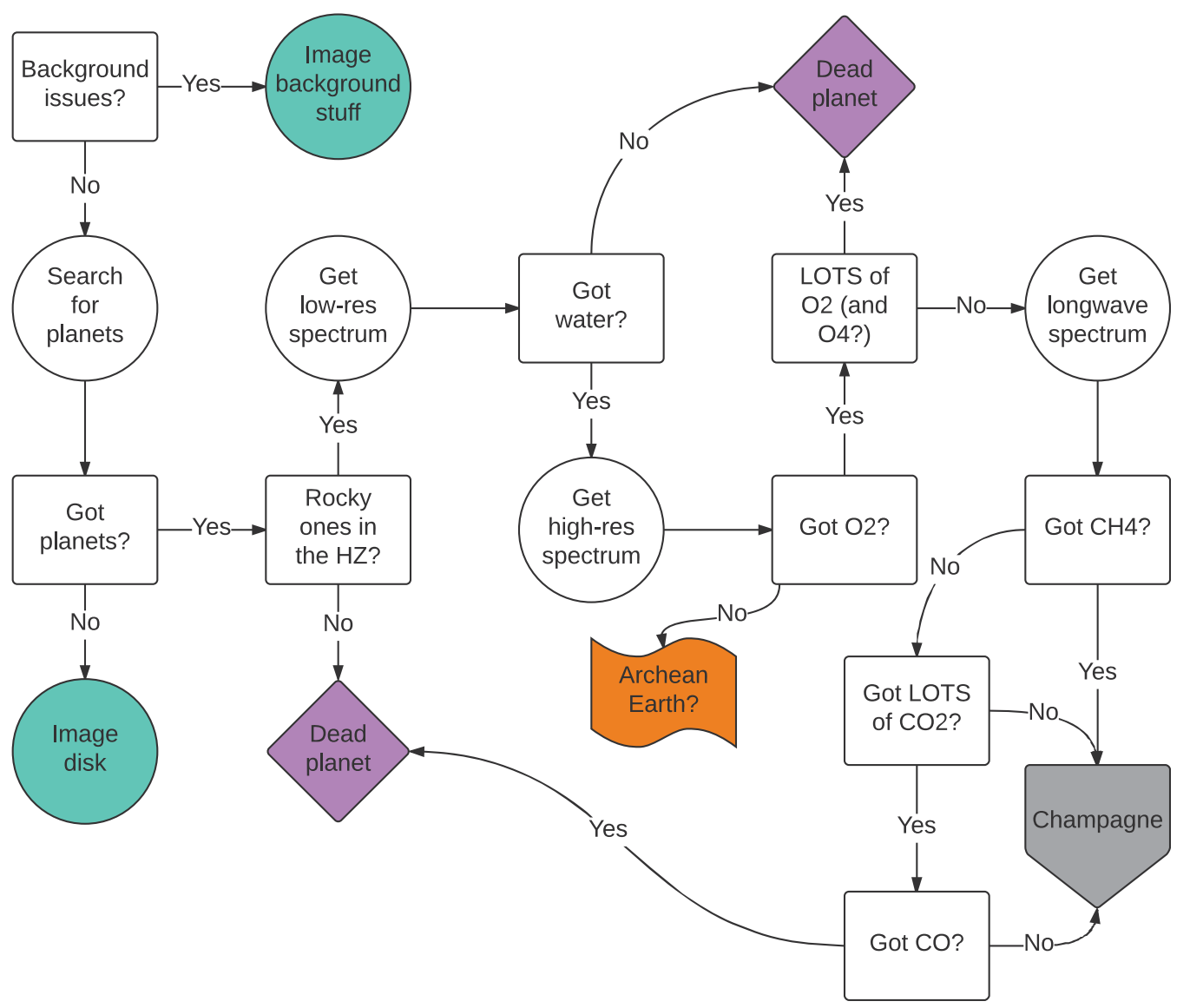

FIG. 11. A flowchart cartoon of possible steps to be taken in searching for a photosynthetic biosphere on an extrasolar planet, and in interpreting detection or nondetection of $\mathrm{O}_{2}$. The process starts with a survey of the background, and includes searching not only for the biosignature $\mathrm{O}_{2}$ but also for other atmospheric molecules such as $\mathrm{CH}_{4}$, which may support the biological interpretation of any $\mathrm{O}_{2}$ observed, and $\mathrm{CO}_{2}$ and $\mathrm{O}_{4}$, which may indicate abiotic processes that could be producing $\mathrm{O}_{2}$ without life. This flowchart also shows how observations of molecules other than $\mathrm{O}_{2}$ can be used to classify environments like the early Earth's, thereby identifying potentially habitable, but anoxic environments. (Credit: Shawn DomagalGoldman).

positive discriminators to different degrees. For direct imaging, a larger planet will require smaller integration times for detection of spectral features, roughly proportional to 1/ $\mathrm{R}_{\mathrm{p}}{ }^{2}$ such that a 1.5 Earth radius super-Earth needs a little less than half the exposure time of an Earth radius planet. Enhanced gravity will decrease the scale height of the planetary atmosphere, as scale height $\mathrm{H}$ is proportional to $1 /$ g. This may allow a planet to emit to space at a higher temperature (Kopparapu et al., 2014) potentially increasing detectability at thermal wavelengths, but will not affect the observability of features at nonthermal wavelengths, assuming the overall surface pressure of the atmosphere remained constant.

For transmission, however, the decrease in scale height due to enhanced gravity would decrease the size of molecular features in the spectrum and, therefore, their detectability. However, the increase in planet radius partially acts to offset this effect. After the scaling relationship for the size of molecule features observed in transmission (Winn, 2010), it can be shown that the size of molecular features in the atmosphere of a super-Earth scales inversely with the ratio of the density of the super-Earth to Earth. If we again assume a super-Earth with a 1.5 Earth's radius, and a mass of
4 Earth masses, then the size of molecular features in the transmission spectrum would be $84 \%$ that of an Earth-sized planet of similar atmospheric composition.

\section{An Integrated Observing Strategy for Finding and Identifying an Oxygenic Photosynthetic Biosphere}

The broad steps in a potential observing strategy to identify $\mathrm{O}_{2}$-bearing, inhabited terrestrial planets include detecting the planet in the presence of astronomical noise sources, preliminary characterization of the planet, detection of $\mathrm{O}_{2}$, the search for false positives, and using the combination of planetary environmental characteristics and the results of the false positive searches to discriminate between biotic and abiotic sources for $\mathrm{O}_{2}$. These steps for a spacebased direct imaging telescope are illustrated in Figure 11, including discriminating an $\mathrm{O}_{2}$-bearing world from a habitable, but anoxic, world like the early Earth. For these steps, we specifically assume that spatially resolved spectra over the field of view are possible, and that the mission can observe in the UV-NIR wavelength range. Other observing strategies may be more appropriate for observatories without these capabilities. Although this strategy will be most 
appropriate for a telescope concept such as LUVOIR or HabEx, a similar or complementary approach to characterizing planetary environmental context and searching for biosignatures could be developed for MIR-FIR direct imaging, or for transit spectroscopy.

Note that we assume that the target stars are well characterized before the planet search, including, if possible, measurements of UV radiation and stellar activity, especially if the mission is not capable of obtaining stellar characterization measurements itself. For missions with UV spectroscopic capabilities, observations of the star bracketing the exoplanet observations would also be particularly valuable for interpretation of the planet's photochemistry, and its biosignatures (e.g., Segura et al., 2005, 2010).

It is also important to note that a large direct imaging telescope that will observe nearby $(<20 \mathrm{pc})$ habitable zone Earth-sized planets predominantly orbiting F-K dwarfs will have to search for planetary targets as part of the initial mission activities. This is because nearby nontransiting planets orbiting more Sun-like stars have yet to be found by existing observations and surveys. Instead, these surveys have either found Earth-sized planets transiting distant (8001000 pc) stars (e.g., Kepler), or orbiting nearby late type M dwarfs (ground-based surveys, e.g., Berta-Thompson et al., 2015), where the discovered planets may be far too close to their star to be observed in direct imaging (e.g., TRAPPIST1; Gillon et al., 2017). The space-based astrometric Gaia mission does not have the precision to detect terrestrial planets (Sozzetti, 2014). Finding nontransiting Earth-sized planets around nearby stars with current ground-based RV searches is extremely challenging, and also favors $M$ dwarf candidates, with the most promising discovery so far being Proxima Centauri b (Anglada-Escudé et al., 2016).

For upcoming missions, the transiting exoplanet survey satellite (Ricker et al., 2015) may also find $\sim 10$ nearby planets transiting nearby planets transiting M dwarfs (Sullivan et al., 2015), and the ESA PLATO mission will search for transiting planets orbiting $\mathrm{G}$ and $\mathrm{K}$ dwarfs (Rauer et al., 2014). However, the probability of finding a transiting Earth around a G dwarf is 1 in 200, and there are only $20 \mathrm{G}$ dwarfs within $10 \mathrm{pc}$. So although there may be a handful of suitable targets for direct imaging (most likely orbiting $\mathrm{K}$ dwarfs) found by transit or RV observations before the launch of large direct imaging telescopes, the majority of habitable zone planets characterized by large direct-imaging telescopes will have also been discovered by these missions in their first 1-2 years of operation.

\subsection{Planet detection}

As discussed, one of the first challenges will be to detect the planet and discriminate it from potential background sources, which include "exozodiacal dust" ("exozodi") from the planetary system dust disk, and background stars and galaxies. This could be done either with multiepoch photometric observations-revisits to the planetary system spread out over a longer time period-or with multiwavelength imaging in a single visit. Multiepoch observations will acquire the position of the planet against the background sky, which will more robustly discriminate the planet from background sources. These observations will constrain the planet's orbit and help determine mass and orbital parameters such as semimajor axis and eccentricity (Stapelfeldt et al., 2015), which are crucial for determining whether the planet is in the habitable zone of its parent star. An alternative, potentially faster method of planet detection would be to use a single visit with multiwavelength imaging to look for point sources near the star. Additional information could then be used to determine whether these sources are more or less likely to be planets or background objects. For example, point sources detected within the exo-zodi are more likely to be planets than sources detected outside the disk. During this detection phase, photometric colors-or low-resolution spatially resolved spectra across the field of view-could enhance our ability to discriminate planets from astrophysical noise sources such as stars, brown dwarfs, and galaxies (Seager et al., 2015), but a definitive determination of whether the planet is in the HZ would not be possible with a single visit.

\subsection{Preliminary characterization}

If the planet is detected in a single visit, then planetary photometric colors could be used to not only discriminate planets from background noise sources but also to attempt to discriminate between different types of terrestrial or more volatile rich (e.g., mini-Neptune) planets. However, planetary environments are rich and complex, especially terrestrial planets that may display inhomogeneous surfaces, volatiles in multiple phases, and a broad diversity of atmospheric compositions. Colors would, therefore, provide only a possibly ambiguous initial classification (e.g., Crow et al., 2011; Livengood et al., 2011; Hegde and Kaltenegger, 2013), because strong degeneracies in environment types that fit a given region of the color-color diagram likely exist (Krissansen-Totton et al., 2016b), and the overlying atmosphere and clouds can modify surface colors (Schwieterman et al., 2015a). Therefore, a potentially more valuable initial method of discriminating between planetary targets would be to obtain low-resolution $(R \sim 10)$ spectra to search for molecules with broad absorption features, such as water vapor and $\mathrm{O}_{3}$ in the visible and NIR spectra. Although neither the $\mathrm{H}_{2} \mathrm{O}$ nor the $\mathrm{O}_{3}$ Chappuis band would be uniquely diagnostic of oceans or life, respectively, the detection of either of these gases would be consistent with habitability and biology. Water vapor features are present near the $\mathrm{O}_{2}$ A-band at 0.72 and $0.85 \mu \mathrm{m}$, and at increasing strength at $0.95,1.14$, and $1.4 \mu \mathrm{m}$.

If multiepoch photometric/astrometric observations are used for detection, then the initial characterization could include determining the planet's orbit and, therefore, semimajor axis and eccentricity. These parameters will help determine the planet's insolation, which will constrain climate models of the planet, and indicate whether or not the planet is in the habitable zone. Mass determination may be done by using or combining any follow-up or existing RV data with the observed position of the planet. The orientation of the dust disk may also help break the RV degeneracy between mass and inclination of the planetary system, although an orbital solution for the planet will do this also. At this stage, direct constraints on planetary radius will only be possible if the planet transits, or if ground- or space-based MIR observations are able to observe the target (e.g., Quanz et al., 2015). Size or mass may help indicate planets that are 
more likely to be terrestrial (Rogers, 2015; Fulton et al., 2017), and size and mass will constrain its density.

Time-resolved, multiwavelength photometry, even in the limited wavelength range of a single $20 \%$ coronagraphic bandpass, and for a single visit, could be used to map the planet and search for surface inhomogeneities that may indicate a terrestrial planet (Cowan et al., 2009; Kawahara and Fujii, 2010; Lustig-Yaeger et al., 2017). This mapping could also take advantage of time-resolved segments of a longexposure spectroscopic observation whose primary purpose is to search for atmospheric constituents (Lustig-Yaeger et al., 2017). For multiepoch observations, photometry at NIR wavelengths (optimally at $0.8-0.9 \mu \mathrm{m}$, and/or between water bands in the $1.0-1.4 \mu \mathrm{m}$ region) comparing brightness at different points in the planet's orbit near gibbous and crescent phases could be used to search for ocean glint (Robinson et al., 2010, 2014). Glint observations may be challenging; observing the planet when half-illuminated produces a maximum separation between star and planet, but the crescent phase occurs at a close apparent separation between star and planet, when the planet is, therefore, more likely to be interior to the IWA. In addition, the inclination of the orbit of the planet to the observer must be closer to edge-on than face-on, otherwise glint will not be observed. Even so, LUVOIR-class missions with $16 \mathrm{~m}$ mirrors may be able to search for glint for a subset of their terrestrial planets (Meadows et al., 2018). Detection of any of these planetary characteristics - terrestrial mass or size, interesting maps, glint, or hints at the presence of water or $\mathrm{O}_{3}$ - would be enough to motivate further observations at higher spectral resolutions $(R \sim 150)$.

\subsection{The search for an oxygenic photosynthetic biosphere with high-resolution spectroscopy}

Once a promising target has been identified, significant time could be invested in obtaining a high-resolution spectrum. At an $R$ of $\sim 150$, individual features from many molecules can be detected. $\mathrm{O}_{2}$ should be sought, and if detected, deeper observations should be taken to constrain its concentration. The $\mathrm{O}_{2} \mathrm{~A}$-band at $0.76 \mu \mathrm{m}$ is a narrow but deep feature at Earthlike concentrations, and the wavelength range and spectral resolution of potential future direct imaging observatories such as HabEx and LUVOIR will be designed to detect it in the atmospheres of our exoplanetary neighbors. Detecting the $\mathrm{O}_{3}$ UV Hartley band $(0.2-0.3 \mu \mathrm{m})$ may reveal the presence of $\mathrm{O}_{2}$ when $\mathrm{O}_{2}$ concentrations are otherwise too low to produce observable spectral features. This may be the case for planets like the Proterozoic Earth, where its $0.1 \%$ PAL $_{2}$ is likely not detectable in reflected light in the visible-NIR spectra with currently anticipated instrumentation, but the $\mathrm{O}_{3}$ Hartley band produces a deep feature for $\lambda<0.3 \mu \mathrm{m}$ (Fig. 3). As stars are fainter in the UV spectrum, longer integration times, wider bandpasses, or both, will be required to obtain high $\mathrm{S} / \mathrm{N}$ observations of UV absorption features. The 30-h observation of an Earth twin planet at $10 \mathrm{pc}$ with a $15 \mathrm{~m}$ telescope shown in Figure 6 returned adequate $\mathrm{S} / \mathrm{N}$ in the $\mathrm{UV}$, but for a spectral resolution of 20, significantly lower than the $R=150$ assumed for the visible channel. If $\mathrm{O}_{2}$ or $\mathrm{O}_{3}$ is not detected, but $\mathrm{H}_{2} \mathrm{O}$ is, this may not indicate a lifeless planet, but rather one with a different dominant metabolism (e.g., methane-producing), or an environment that leads to a false negative, as would have been the case for the early Earth.

\subsection{Further characterization and elimination of false positives}

Other molecules should be sought to characterize the planetary environment, including $\mathrm{O}_{3}, \mathrm{O}_{4}, \mathrm{CO}, \mathrm{CO}_{2}$, and $\mathrm{CH}_{4}$, as well as clouds and aerosols. The presence of $\mathrm{O}_{4}$, especially for a planet orbiting a late-type $\mathrm{M}$ dwarf, may indicate that any $\mathrm{O}_{2}$ present is the result of ocean loss and $\mathrm{H}$ escape due to $\mathrm{H}_{2} \mathrm{O}$ photolysis (Luger and Barnes, 2015; Schwieterman et al., 2016). In direct imaging, the strong $\mathrm{O}_{4}$ collision-induced absorption features in the visible region $(0.34-0.7 \mu \mathrm{m})$ would likely be detectable in the same observations required to detect the $\mathrm{O}_{2}(0.76 \mu \mathrm{m})$, and could potentially be in the same coronagraphic bandpass. However, for transmission observations, $\mathrm{O}_{4}$ bands in the NIR at 1.06 and $1.27 \mu \mathrm{m}$ would be more detectable than those in the visible, as they are less affected by the increase in atmospheric opacity engendered by Rayleigh scattering at shorter wavelengths. Note also that ground-based high-resolution spectroscopy will not be able to observe the broad features of $\mathrm{O}_{4}$ collisionally induced absorption, and so this false positive discriminator is not available to this technique.

If abundant $\mathrm{CH}_{4}$ is observed with $\mathrm{O}_{2}$, this would make most photochemical false positives less likely, as $\mathrm{CH}_{4}$ is a sink for photochemically generated $\mathrm{O}_{2}$. It is also less likely to build up to detectable levels in the massive $\mathrm{O}_{2}$ atmospheres of ocean loss planets. Thus, detection of $\mathrm{CH}_{4}$ in an atmosphere with $\mathrm{O}_{2}$ features would make ocean loss and photochemical false positives less likely, and suggest a high $\mathrm{O}_{2}$ replenishment rate and a likely biogenic source of the $\mathrm{O}_{2}$. However, $\mathrm{CH}_{4}$ features may be challenging to observe at modern Earth-like concentrations because methane's strongest features are longward of $2.5 \mu \mathrm{m}$, where telescopic thermal emission and IWA issues may preclude observation. Weaker bands in the visible region $(0.7-0.9 \mu \mathrm{m})$ would require abundances of $\mathrm{CH}_{4}$ in excess of those seen in Earth's atmosphere today, but are within the wavelength ranges anticipated for direct imaging spacecraft and ground-based high-resolution spectroscopy (Lovis et al., 2017). Indeed, as described in section 2.2, there may have been no period in Earth's history wherein both $\mathrm{O}_{2}$ and $\mathrm{CH}_{4}$ coexisted in high abundance (Olsen et al., 2016; Reinhard et al., 2017).

Photochemical generation of $\mathrm{O}_{2}$ and/or $\mathrm{O}_{3}$ from $\mathrm{CO}_{2}$ photolysis (Hu et al., 2012; Domagal-Goldman et al., 2014; Gao et al., 2015; Harman et al., 2015) should be associated with high amounts of $\mathrm{CO}_{2}$ and $\mathrm{CO}$ in the planetary atmosphere. $\mathrm{CO}_{2}$ has a weak band near $1.6 \mu \mathrm{m}$, which is undetectable on modern Earth but would likely have a strong feature for atmospheres with enough $\mathrm{CO}_{2}$ to produce $\mathrm{O}_{2}$ or $\mathrm{O}_{3}$ photochemically (Domagal-Goldman et al., 2014). $\mathrm{CO}_{2}$ has a much stronger band at $4.2 \mu \mathrm{m}$, although the latter band will be difficult to access for warm telescopes (either ground-based or actively heated space-based telescopes) dominated by thermal radiation in this spectral region. $\mathrm{CO}$ also absorbs near 2.35 and $4.6 \mu \mathrm{m}$, where a warm (room temperature) mirror will also preclude observation. In addition, these longer wavelengths will require a large telescope diameter to avoid falling within the IWA for observatories cold enough to see them, or an extremely large starshade to provide suppression at those longer wavelengths. 
Transit transmission observations, however, from the ground and JWST may allow access to longer wavelengths in the near term, compared with direct imaging techniques, and groundbased observations may also be attempted for the $2.35 \mu \mathrm{m} \mathrm{CO}$ band (c.f. Snellen et al., 2013).

\subsection{Detailed planet characterization and the search for secondary biosignatures}

If all known false positive mechanisms for the presence of $\mathrm{O}_{2}$ have been eliminated, and if photochemical models and the presence of sinks such as $\mathrm{CH}_{4}$ suggest a high $\mathrm{O}_{2}$ flux into the environment, then this would make a biogenic source more likely. This conclusion can be bolstered not only by ruling out false positives, but also by searching for secondary confirmation of the dominant metabolism that is believed to have been discovered. In the case of oxygenic photosynthesis, this could come in the form of secondary biosignatures such as detection of a red edge or other pigment-associated feature in multiwavelength, time-resolved photometric mapping (LustigYaeger, 2017) or in seasonally varying concentrations of $\mathrm{O}_{2}$, $\mathrm{CO}_{2}$, or $\mathrm{CH}_{4}$ (Meadows, 2008; Reinhard et al., 2016), or the detection of other biosignatures such as $\mathrm{N}_{2} \mathrm{O}$ (Schwieterman et al., 2018). Eventually, other biosignatures and their false positives will be identified and sought, and the measurements obtained from any single planet will be placed in the broader context of similar measurements made on other worlds (Fujii et al., 2018; Walker et al., 2018), further informing our understanding of comparative planetology, and the prevalence of life in the Universe.

\section{Discussion and Lessons Learned}

The recent detailed study of $\mathrm{O}_{2}$ has advanced the field of biosignatures by providing an in-depth exploration of the impact a planetary environment can have on our ability to detect and recognize biosignatures. $\mathrm{O}_{2}$ can, therefore, serve as an exemplar for a more generalized framework for biosignature detection, and this concept is further developed for other biosignatures in the work of Catling et al. (2018).

$\mathrm{O}_{2}$ has long been considered an excellent biosignature, in part, because of its characteristics in the modern Earth environment. $\mathrm{O}_{2}$ is an abundant gas that is stable against photolysis, and so it is evenly mixed throughout the atmosphere. This increases its detectability even in the presence of clouds, or when using techniques such as transmission spectroscopy that do not probe deep into the planetary atmosphere (Meadows, 2017). In addition, for Earth, there are no known abiotic processes that would produce it in large abundance.

The study of $\mathrm{O}_{2}$ as a biosignature draws heavily on our understanding of how Earth and life coevolved over time, and the planetary processes that may have suppressed or enabled oxygen's rise from a trace gas to one of the principal components of our planetary atmosphere (Lyons et al., 2014). We have learned that a biosignature will not build up to detectable levels in a planetary atmosphere simply because life evolved, but rather as a result of the complex interactions of life with the planet and the host star, often for billions of years. The study of $\mathrm{O}_{2}$ throughout Earth's history has introduced the concept of a false negative (e.g., Reinhard et al., 2017), wherein life is present, but its mark on the environment is unseen.

Similarly, the field of exoplanets has revealed stars and worlds, with evolutionary sequences that are likely very different to Earth's around the Sun. By modeling these environments and their interactions, researchers have opened up a possibility that was previously unthinkable for an Earth-like environment-that $\mathrm{O}_{2}\left(\right.$ or $\left.\mathrm{O}_{3}\right)$ could be generated in a planetary environment without the action of life (e.g., $\mathrm{Hu}$ et al., 2012; Domagal-Goldman et al., 2014; Wordsworth and Pierrehumbert, 2014; Gao et al., 2015; Harman et al., 2015; Luger and Barnes, 2015; Narita et al., 2015; Tian, 2015).

Our improved understanding of false negatives for $\mathrm{O}_{2}$ may help us optimize target selection for the search for life on exoplanets, and it points to a rich area for future work. Being able to assess a target's false negative potential could be an integral part of the search for life, and it is an important foundation for interpreting what we do (and do not) detect. To advance this area of research, we will need to develop an improved understanding of planetary and stellar characteristics that may lead to false negatives, and the observational discriminants that might reveal them.

As specific examples, $\mathrm{O}_{2}$ may have only overwhelmed (or outlasted) its environmental sinks on Earth fairly recently, and so perhaps it would be prudent to prioritize older planets to allow time for a photosynthetic metabolism to develop and leave its footprint. The interior and crustal composition of the planet may make it more likely for reducing gases to be present. Understanding how observed stellar composition affects terrestrial planet composition (e.g., Young et al., 2014; Dorn et al., 2015; Unterborn and Panero, 2017), how planetary orbit and star may affect tidal heating, interior structure, and surface heat flow (e.g., Driscoll and Barnes, 2015), and searching for the presence of reducing gases or tectonic/volcanic activity through atmospheric gases or aerosols (e.g., Kaltenegger and Sasselov, 2009; Kaltenegger et al., 2010; Misra et al., 2015), may all be important for constraining possible sinks for $\mathrm{O}_{2}$. Similarly, understanding the incoming stellar UV spectrum and activity levels and the overall impact on atmospheric chemistry and photolysis rates would also help identify processes that may enhance or suppress the detectability of a biosignature (e.g., Segura et al., 2003, 2005; Grenfell et al., 2007, 2014; Rugheimer et al., 2013, 2015). In this example, young volcanically active planets with strongly reducing atmospheres may not represent good targets for the search for oxygenic photosynthesis.

Our improved understanding of the star-planet processes that may lead to false positives for $\mathrm{O}_{2}$ has only strengthened the robustness of $\mathrm{O}_{2}$ as a biosignature. This knowledge can also inform our target selection, and prepare us to observe planetary characteristics that will help discriminate whether observed $\mathrm{O}_{2}$ has a biological or abiotic origin. We now know of several abiotic planetary processes that will generate $\mathrm{O}_{2}$ or $\mathrm{O}_{3}$ for planets orbiting $\mathrm{M}$ dwarfs (Wordsworth and Pierrehumbert, 2014; Domagal-Goldman et al., 2014; Tian et al., 2014; Harman et al., 2015; Luger and Barnes, 2015; Tian, 2015). There are very likely more, and the search should continue for additional false positive mechanisms using modeling efforts or observations of exo-Venuses (Kane et al., 2014; Berta-Thompson et al., 2015; Gillon et al., 2017), which may provide observational tests for some of the proposed mechanisms. In particular, planetary environmental modeling is critically needed to anticipate the challenges we will face in interpreting the spectra obtained by future observatories. 
Identification of potential false positives helps guide recommendations for the wavelength ranges and spectral resolutions needed to discriminate them, and allows us to design a more sophisticated and robust observing sequence for the search for life to exoplanets. We now know that the detection of any potential biosignature gas in an exoplanet's atmosphere must be considered in the context of the complex web of interrelated processes that shape the broader planetary environment. For example: where is the planet relative to its star's habitable zone? What is the activity level of the star, and what types of photochemistry can it drive? Does the planet show other signs of habitability such as liquid surface water from water vapor or glint? What other gases are present? What fluxes are required to maintain the observed concentrations of the biosignatures gas? What false positive mechanisms may explain the observed possible biosignatures? Are there other potential biosignatures present?

If $\mathrm{O}_{2}$ is detected, other characteristics of the planet can be observed to rule out false positive mechanisms, and to bolster the likelihood that the $\mathrm{O}_{2}$ comes from a planetary biosphere. Choosing an older planet would again not only decrease the probability of a false negative but decrease the likelihood of a false positive as well, as an initially high atmospheric $\mathrm{O}_{2}$ abundance from ocean loss becomes sequestered or lost to space (Chassefière, 1996; Schaefer et al., 2016).

Searching for a combination of gases or planetary characteristics, and not finding some of them, could also be powerful. Detecting $\mathrm{O}_{2}$ and water or ocean glint, without $\mathrm{O}_{4}$, can be more robust than $\mathrm{O}_{2}$ alone. Detecting $\mathrm{O}_{2}, \mathrm{CH}_{4}$, and water, or detecting $\mathrm{O}_{2}$ and water without strong $\mathrm{CO}_{2}$ or $\mathrm{CO}$, are both stronger detections. Finally, searching for secondary biosignatures of photosynthesis such as the red edge (Gates et al., 1965) and seasonal variability (Meadows, 2008; Reinhard et al., 2016) also strengthens the case for $\mathrm{O}_{2}$ as a biosignature. Searching for the multiple planetary characteristics that strengthen the likelihood that $\mathrm{O}_{2}$ is a biosignature requires observations that span the broadest possible spectral range, and that are tailored for the strengths of the observing mode chosen, whether that is direct imaging, transmission, or high-resolution spectroscopy.

In closing, oxygenic photosynthesis evolved a metabolism that harvested light from the parent star to split water and fix carbon, and it ultimately rose to sculpt the history of our planet and produce the strongest biosignatures on Earth today. However, the absence of $\mathrm{O}_{2}$ throughout much of Earth's history also encourages us to learn more about biosignatures that are not tied to oxygenic photosynthesis, to be better prepared for environments in which oxygenic photosynthesis has either not evolved or not yet overwhelmed sinks for $\mathrm{O}_{2}$. Yet for any new biosignatures that are identified, the same process that has been developed for the study of $\mathrm{O}_{2}$ should still be applied. Biosignatures cannot exist distinct from the environment that harbors them. Understanding the impact of the environment on the detectability of biosignatures, and the potential generation of false positives, will be an important activity for future biosignature research.

\section{Conclusions}

The study of $\mathrm{O}_{2}$ has catalyzed the interdisciplinary synthesis of research in early Earth science with the modeling of star-planet interactions in exoplanet science, to greatly expand our understanding of biosignatures in the context of their environments. The early simplistic view that $\mathrm{O}_{2}$ alone constituted the most robust biosignature for detection of life on exoplanets has given way to a more sophisticated understanding of the impact of a planetary environment on the detectability and interpretation of $\mathrm{O}_{2}$ in a planetary spectrum. The delay between the advent of oxygenic photosynthesis on Earth and the rise of atmospheric $\mathrm{O}_{2}$ to modern levels reveal a false negative process where life is present, but not detectable, due to suppression of the biosignature by the planetary environment. Future research into false negative processes may reveal planetary processes and observational discriminants that could help identify planets on which biosignatures are likely suppressed. This could potentially support exoplanet target selection for biosignature searches and inform the interpretation of observed planetary spectra. Similarly, the study of false positives has revealed stellar and planetary characteristics that may cause $\mathrm{O}_{2}$ to build up abiotically in a planetary environment and identified observational discriminants for those processes. This allows observations of $\mathrm{O}_{2}$ in a planetary spectrum to be more robustly interpreted as a biosignature by searching for and ruling out false positive mechanisms. The processes to identify false negatives and positives for $\mathrm{O}_{2}$ serve as an exemplar for a more generalized process for biosignature detection that should be applied to other novel biosignatures.

\section{Acknowledgments}

We thank the NASA Astrobiology Program and the Nexus for Exoplanet System Science (NExSS) for their support for the NExSS Exoplanet Biosignatures Workshop. Conversations at this workshop, held in the northern hemisphere summer of 2016, formed the basis for the drafting of the five review articles in this issue. We thank Mary Voytek, the senior scientist for Astrobiology, for her leadership of NExSS, without whom this interdisciplinary community synthesis would not have been possible, and her feedback on our organization of the workshop and articles. We would like to thank Tanai Cardona and Mercedes Lopez-Morales who provided helpful comments on a draft of this article during the NExSS-hosted open community discussion forum. We thank two anonymous reviewers for thoughtful comments that helped us improve this article. V.S.M., G.N.A., M.N.P., S.D.-G., E.W.S., A.P.L., J.L.Y., and R.D. were supported by NASA Astrobiology Institute's Virtual Planetary Laboratory under Cooperative Agreement Number NNA13AA93A. C.T.R., E.W.S., and T.W.L. received support from the NASA Astrobiology Institute Alternative Earths team under Cooperative Agreement Number NNA15BB03A. S.D.S. acknowledges support from NASA Exobiology grant NNX15AM07G. E.W.S. is also grateful for support from the NASA Postdoctoral Program, administered by the Universities Space Research Association.

\section{Author Disclosure Statement}

No competing financial interests exist.

\section{References}

Airapetian, V.S., Glocer, A., Gronoff, G., Hébrard, E., and Danchi, W. (2016) Prebiotic chemistry and atmospheric warming of early Earth by an active young Sun. Nat Geosci 9:52. 
Airapetian, V.S., Glocer, A., Khazanov, G.V., Loyd, R.O.P., France, K., Sojka, J., Danchi, W.C., and Liemohn, M.W. (2017) How hospitable are space weather affected habitable zones? The role of ion escape. Astrophys J Lett 836:L3.

Allen, J.P. and Williams, J.C. (1998) Photosynthetic reaction centers. FEBS Lett 438:5-9.

Anbar, A.D., Duan, Y., Lyons, T.W., Arnold, G.L., Kendall, B., Creaser, R.A., Kaufman, A.J., Gordon, G.W., Scott, C., Garvin, J., and Buick, R. (2007) A whiff of oxygen before the great oxidation event? Science (New York, N.Y.) 317:19031906.

Anglada-Escudé, G., Amado, P.J., Barnes, J., Berdiñas, Z.M., Butler, R.P., Coleman, G.A.L., de la Cueva, I., Dreizler, S., Endl, M., Giesers, B., Jeffers, S.V., Jenkins, J.S., Jones, H.R.A., Kiraga, M., Kürster, M., López-González, M.J., Marvin, C.J., Morales, N., Morin, J., Nelson, R.P., Ortiz, J.L., Ofir, A., Paardekooper, S.-J., Reiners, A., Rodríguez, E., Rodríguez-López, C., Sarmiento, L.F., Strachan, J.P., Tsapras, Y., Tuomi, M., and Zechmeister, M. (2016) A terrestrial planet candidate in a temperate orbit around Proxima Centauri. Nature 536:437-440.

Arney, G., Domagal-Goldman, S.D., Meadows, V.S., Wolf, E.T., Schwieterman, E., Charnay, B., Clare, M., Hébrard, E., and Trainer, M.G. (2016) The pale orange dot: the spectrum and habitability of hazy Archean earth. Astrobiology 16:873899.

Arney, G.N., Meadows, V.S., Domagal-Goldman, S.D., Deming, D., Robinson, T.D., Tovar, G., Wolf, E.T., and Schwieterman, E. (2017) Pale orange dots: the impact of organic haze on the habitability and detectability of earthlike exoplanets. Astrophys $J$ 836:49.

Baraffe, I., Chabrier, G., Allard, F., and Hauschildt, P. (1998) Evolutionary models for solar metallicity low-mass stars: mass-magnitude relationships and color-magnitude diagrams. A\&A 337:403-412.

Barnes, R., Deitrick, R., Luger, R., Driscoll, P.E., Quinn, T.R., Fleming, D.P., Guyer, B., McDonald, D.V., Meadows, V.S., Arney, G., Crisp, D., Domagal-Goldman, S.D., Lincowski, A., Lustig-Yaeger, J., and Schwieterman, E. (2018) The habitability of Proxima Centauri b I: evolutionary scenarios. arXiv:1608.06919v2.

Barstow, J.K., Aigrain, S., Irwin, P.G., Fletcher, L.N., and Lee, J.M. (2013). Constraining the atmosphere of GJ 1214b using an optimal estimation technique. MNRAS 434:2616-2628.

Barstow, J.K., Aigrain, S., Irwin, P.G., Kendrew, S., and Fletcher, L.N. (2015) Transit spectroscopy with James Webb Space Telescope: systematics, starspots and stitching. MNRAS 448: 2546-2561. Chicago.

Bekker, A., Holland, H.D., Wang, P.-L., Rumble, D., Stein, H.J., Hannah, J.L., Coetzee, L.L., and Beukes, N.J. (2004) Dating the rise of atmospheric oxygen. Nature 427:117-120.

Bell, E.A., Boehnke, P., Hopkins-Wielicki, M.D., and Harrison, T.M. (2015) Distinguishing primary and secondary inclusion assemblages in Jack Hills zircons. Lithos 234:15-26.

Benneke, B. and Seager, S. (2012) Atmospheric retrieval for super-earths: uniquely constraining the atmospheric composition with transmission spectroscopy. Astrophys $J$ 753:100.

Berner, R.A. (1982) Burial of organic carbon and pyrite sulfur in the modern ocean: its geochemical and environmental significance. Am J Sci 282:451-473.

Berner, R.A. (2004) The Phanerozoic Carbon Cycle: $\mathrm{CO}_{2}$ and $\mathrm{O}_{2}$. Oxford University Press, New York.
Berner, R.A. (2006) GEOCARBSULF: a combined model for Phanerozoic atmospheric $\mathrm{O}_{2}$ and $\mathrm{CO}_{2}$. Geochim Cosmochim Acta 70:5653-5664.

Berta-Thompson, Z.K., Irwin, J., Charbonneau, D., Newton, E.R., Dittmann, J.A., Astudillo-Defru, N., Bonfils X, Gillon M, Jehin E, Stark AA, Stalder B, Bouchy F, Delfosse X, Forveille T, Lovis C, Mayor M, Neves V, Pepe F, Santos NC, Udry S, and Wünsche, A. (2015) A rocky planet transiting a nearby low-mass star. Nature 527:204-207.

Bétrémieux, Y. and Kaltenegger, L. (2013) Transmission spectrum of earth as a transiting exoplanet from the ultraviolet to the near-infrared. Astrophys J Lett 772:L31.

Bétrémieux, Y. and Kaltenegger, L. (2014) Impact of atmospheric refraction: how deeply can we probe exo-earth's atmospheres during primary eclipse observations? ApJ 791:7.

Bolcar, M.R., Balasubramanian, K., Clampin, M., Crooke, J., Feinberg, L., Postman, M., Quijada, M., Rauscher, B., Redding, D., Rioux, N., Shaklan, S., Stahl, H.P., Stahle, C., and Thronson, H. (2015) Technology development for the Advanced Technology Large Aperture Space Telescope (ATLAST) as a candidate large UV-Optical-Infrared (LUVOIR) surveyor. In UV/Optical/IR Space Telescopes: Innovative Technologies and Concepts VII, Proc. SPIE, edited by H.A. MacEwen, and J.B. Breckinridge, p 960209. DOI: 10.1117/12.2188559

Brandl, B.R., Feldt, M., Glasse, A., Guedel, M., Heikamp, S., Kenworthy, M., Lenzen, R., Meyer, M.R., Molster, F., Paalvast, S., Pantin, E.J., Quanz, S.P., Schmalz, E., Stuik, R., Venema, L., and Waelkens, C. (2014) METIS: the midinfrared E-ELT imager and spectrograph. In Ground-Based and Airborne Instrumentation for Astronomy V, Proc. SPIE 914721.

Buick, R., Dunlop, J.S.R., and Groves, D.I. (1981) Stromatolite recognition in ancient rocks: an appraisal of irregularly laminated structures in an Early Archaean chert-barite unit from North Pole, Western Australia. Alcheringa 5:161-181.

Bryant, D.A., Costas, A.M.G., Maresca, J.A., Chew, A.G.M., Klatt, C.G., Bateson, M.M., Tallon, L.J., Hostetler, J., Nelson, W.C., Heidelberg, J.F., and Ward, D.M. (2007) Candidatus Chloracidobacterium thermophilum: an aerobic phototrophic acidobacterium. Science 317:523-526.

Buick, R. (2007) Did the Proterozoic 'Canfield Ocean' cause a laughing gas greenhouse? Geobiology 5:97-100.

Canfield, D.E. (2005) The early history of atmospheric oxygen: homage to Robert M. Garrels. Ann Rev Earth Planet Sci 33: $1-36$.

Canfield, D.E., Rosing, M.T., and Bjerrum, C. (2006) Early anaerobic metabolisms. Phil Trans R Soc B 361:1819-1836.

Cardona, T. (2015) A fresh look at the evolution and diversification of photochemical reaction centers. Photosynth Res 126:111-134.

Cardona, T. (2016) Reconstructing the origin of oxygenic photosynthesis: do assembly and photoactivation recapitulate evolution? Front Plant Sci 7:257.

Cardona, T. (2017) Photosystem II is a chimera of reaction centers. J Mol Evol 84:149-151.

Carroll, S.B. (2001) Chance and necessity: the evolution of morphological complexity and diversity. Nature 409:11021109.

Catling, D. and Kasting, J.F. (2007) Planetary atmospheres and life. In Planets and Life: The Emerging Science of Astrobiology, edited by W.T. Sullivan and J. Baross, Cambridge University Press, Cambridge, UK, pp 91-116. 
Catling, D.C., Zahnle, K.J., and McKay, C.P. (2001) Biogenic methane, hydrogen escape, and the irreversible oxidation of early Earth. Science 293:839-843.

Catling, D.C., Glein, C.R., Zahnle, K.J., and McKay, C.P. (2005) Why $\mathrm{O}_{2}$ is required by complex life on habitable planets and the concept of planetary "oxygenation time." Astrobiology 5: 415-438.

Catling, D.C., J. Krissansen-Totton, J., Kiang, N.Y., Crisp, D., Robinson, T.D., DasSarma, S., Rushby, A.J., Del Genio, A., Bains, W., and Domagal-Goldman, S. (2018) Exoplanet biosignatures: a framework for their assessment. Astrobiology 18:709-738.

Chassefière, E. (1996) Hydrodynamic escape of hydrogen from a hot water-rich atmosphere: the case of Venus. J Geophys Res Planets 101:26039-26056.

Cole, D.B., Reinhard, C.T., Wang, X., Gueguen, B., Halverson, G.P., Gibson, T., Hodgskiss, M.S., McKenzie, N.R., Lyons, T.W., and Planavsky, N.J. (2016) A shale-hosted Cr isotope record of low atmospheric oxygen during the Proterozoic. Geology 44:555-558.

Collinson, G.A., Frahm, R.A., Glocer, A., Coates, A.J., Grebowsky, J.M., Barabash, S., Domagal-Goldman, S.D., Fedorov, A., Futaana, Y., Gilbert, L.K., and Khazanov, G. (2016) The electric wind of Venus: a global and persistent "polar wind"-like ambipolar electric field sufficient for the direct escape of heavy ionospheric ions. Geophys Res Lett 43:5926-5934.

Cowan, N.B., Agol, E., Meadows, V.S., Robinson, T., Livengood, T.A., Deming, D., Lisse, C.M., A'Hearn, M.F., Wellnitz, D.D., Seager, S., and Charbonneau, D. (2009) Alien maps of an ocean-bearing world. Astrophys J 700:915.

Cowan, N.B., Greene, T., Angerhausen, D., Batalha, N.E., Clampin, M., Colón, K., Crossfield, I.J.M., Fortney, J.J., Gaudi, B.S., Harrington, J., and Iro, N. (2015) Characterizing transiting planet atmospheres through 2025. Publ Astron Soc Pac 127:311.

Cowan, N.B. and Strait, T.E. (2013) Determining reflectance spectra of surfaces and clouds on exoplanets. ApJ 765:L17.

Cox, C. and Munk, W. (1954) Measurement of the roughness of the sea surface from photographs of the sun's glitter. JOSA 44:838-850.

Crossfield, I.J. (2016) Exoplanet atmospheres and giant groundbased telescopes. arXiv preprint arXiv:1604.06458.

Crow, C.A., McFadden, L.A., Robinson, T., Meadows, V.S., Livengood, T.A., Hewagama, T., Barry, R.K., Deming, L.D., Lisse, C.M., and Wellnitz, D. (2011) Views from EPOXI: colors in our solar system as an analog for extrasolar planets. Astrophys J 729:130.

Crowe, S.A., Døssing, L.N., Beukes, N.J., Bau, M., Kruger, S.J., Frei, R., and Canfield, D.E. (2013) Atmospheric oxygenation three billion years ago. Nature 501:535-538.

Czaja, A.D., Johnson, C.M., Roden, E.E., Beard, B.L., Voegelin, A.R., Nägler, T.F., Beukes, N.J., and Wille, M. (2012) Evidence for free oxygen in the Neoarchean ocean based on coupled iron-molybdenum isotope fractionation. Geochim Cosmochim Acta 86:118-137.

Dalcanton, J., Seager, S., Aigrain, S., Hirata, C., Battel, S., Mather, J., Brandt, N., Postman, M., Conroy, C., Redding, D., Feinberg, L., Schiminiovich, D., Gezari, S., Stahl, H.P., Guyon, O., Tumilinson, J., and Harris, W. (2015) From Cosmic Birth to Living Earths: the Future of UVOIR Space Astronomy. From Cosmic Births to Living Earths Report. Association for Research in Astronomy, Washington, DC. www.hdstvision.org/report.

de Bergh, C., Bezard, B., Owen, T., Crisp, D., Maillard, J.P., and Lutz, B.L. (1991) Deuterium on venus: observations from earth. Science 251:547-549.
Deming, D., Seager, S., Winn, J., Miller-Ricci, E., Clampin, M., Lindler, D., Greene, T., Charbonneau, D., Laughlin, G., Ricker, G., Latham, D., and Ennico, K. (2009) Discovery and characterization of transiting super earths using an all-sky transit survey and follow-up by the James Webb Space Telescope. PASP 121:952-967.

Des Marais, D.J., Harwit, M.O., Jucks, K.W., Kasting, J.F., Lin, D.N.C., Lunine, J.I., Schneider, J., Seager, S., Traub, W.A., and Woolf, N.J. (2002) Remote sensing of planetary properties and biosignatures on extrasolar terrestrial planets. Astrobiology 2:153-181.

Des Marais, D.J., Strauss, H., Summons, R.E., and Hayes, J.M. (1992) Carbon isotope evidence for the stepwise oxidation of the Proterozoic environment. Nature v359:p605-p609.

de Wit, J., Wakeford, H.R., Gillon, M., Lewis, N.K., Valenti, J.A., Demory, B.-O., Burgasser, A.J., Burdanov, A., Delrez, L., Jehin, E., Lederer, S.M., Queloz, D., Triaud, A.H., and Van Grootel, V. (2016) A combined transmission spectrum of the Earth-sized exoplanets TRAPPIST-1 b and c. Nature 537: 69-72.

Dodd, M.S., Papineau, D., Grenne, T., Slack, J.F., Rittner, M., Pirajno, F., O'Neil, J., and Little, C.T. (2017) Evidence for early life in Earth's oldest hydrothermal vent precipitates. Nature 543:60-64.

Domagal-Goldman, S.D. and Meadows, V.S. (2010) Abiotic buildup of ozone. Pathways Towards Habitable Planets, Astronomical Society of the Pacific Conference Series 430: 152-157.

Domagal-Goldman, S.D., Meadows, V.S., Claire, M.W., and Kasting, J.F. (2011) Using biogenic sulfur gases as remotely detectable biosignatures on anoxic planets. Astrobiology 11: 419-441.

Domagal-Goldman, S.D., Segura, A., Claire, M.W., Robinson, T.D., and Meadows, V.S. (2014) Abiotic ozone and oxygen in atmospheres similar to prebiotic earth. ApJ 792:43.

Dong, C., Lingam, M., Ma, Y., and Cohen, O. (2017) Is Proxima Centauri b habitable? A study of atmospheric loss. Astrophys J Lett 837:L26.

Dorn, C., Khan, A., Heng, K., Connolly, J.A., Alibert, Y., Benz, W., and Tackley, P. (2015) Can we constrain the interior structure of rocky exoplanets from mass and radius measurements? A\&A 577:A83.

Dressing, C.D. and Charbonneau, D. (2015) The occurrence of potentially habitable planets orbiting $M$ dwarfs estimated from the full Kepler dataset and an empirical measurement of the detection sensitivity. Astrophys $J$ 807:45.

Driese, S.G., Jirsa, M.A., Ren, M., Brantley, S.L., Sheldon, N.D., Parker, D., and Schmitz, M. (2011) Neoarchean paleoweathering of tonalite and metabasalt: implications for reconstructions of $2.69 \mathrm{Ga}$ early terrestrial ecosystems and paleoatmospheric chemistry. Precambrian Res 189:1-17.

Driscoll, P.E. and Barnes, R. (2015) Tidal heating of Earth-like exoplanets around $\mathrm{M}$ stars: thermal, magnetic, and orbital evolutions. Astrobiology 15:739-760.

Etiope, G. and Sherwood Lollar, B. (2013) Abiotic methane on Earth. Rev Geophys 51:276-299.

Falkowski, P., Scholes, R.J., Boyle, E., Canadell, J., Canfield, D., Elser, J., Gruber, N., Hibbard, K., Hogberg, P., Linder, S., Mackenzie, F.T., Moore, B., Pederson, T., Rosenthal, Y., Seitzinger, S., Smetacek, V., and Steffan, W. (2000) The global carbon cycle: a test of our knowledge of earth as a system. Science 290:291-296.

Farquhar, J. (2000) Atmospheric influence of earth's earliest sulfur cycle. Science 289:756-758. 
Farquhar, J., Savarino, J., Airieau, S., and Thiemens, M.H. (2001) Observation of wavelength-sensitive massindependent sulfur isotope effects during $\mathrm{SO}_{2}$ photolysis: Implications for the early atmosphere. $J$ Geophys Res Planets 106:829-839.

Feng, Y.K., Line, M.R., Fortney, J.J., Stevenson, K.B., Bean, J., Kreidberg, L., and Parmentier, V. (2016) The impact of nonuniform thermal structure on the interpretation of exoplanet emission spectra. Astrophys $J$ 829:52.

Field, C.B., Behrenfeld, M.J., Randerson, J.T., and Falkowski, P. (1998) Primary production of the biosphere: integrating terrestrial and oceanic components. Science 281:237-240.

Fortney, J.J. (2005) The effect of condensates on the characterization of transiting planet atmospheres with transmission spectroscopy. MNRAS 364:649-653.

Fujii, Y., Angerhausen, D., Deitrick, R., Domagal-Goldman. S., Grenfell, J.L., Hori, Y., Kane, S.R., Palle, E., Rauer, H., Siegler, N., Stapelfeldt, K., and Stevenson, K.B. (2018) Exoplanet biosignatures: observational prospects. Astrobiology 18: 739-778.

Fulton, B.J., Petigura, E.A., Howard, A.W., Isaacson, H., Marcy, G.W., Cargile, P.A., Hebb, L., Weiss, L.M., Johnson, J.A., Morton, T.D., and Sinukoff, E. (2017) The CaliforniaKepler Survey. III. A Gap in the Radius Distribution of Small Planets. arXiv preprint arXiv:1703.10375.

Gao, P., Hu, R., Robinson, T.D., Li, C., and Yung, Y.L. (2015) Stability of $\mathrm{CO} 2$ atmospheres on dessicated M dwarf exoplanets. ApJ 806:249.

Garcia-Sage, K., Glocer, A., Drake, J.J., Gronoff, G., and Cohen, O. (2017) On the magnetic protection of the atmosphere of Proxima Centauri b. Astrophys J 844:L13.

Gates, D.M., Keegan, H.J., Schleter, J.C. and Weidner, V.R. (1965) Spectral properties of plants. Appl Opt 4:11-20.

Gebauer, S., Grenfell, J.L., Stock, J.W., Lehmann, R., Godolt, M., von Paris, P., and Rauer, H. (2017) Evolution of earth-like extrasolar planetary atmospheres: assessing the atmospheres and biospheres of early earth analog planets with a coupled atmosphere biogeochemical model. Astrobiology 17:27-54.

Gillon, M., Jehin, E., Lederer, S.M., Delrez, L., de Wit, J., Burdanov, A., Van Grootel, V., Burgasser, A., Triaud, A.H.M.J., Opitom, C., Demory, B.-O., Sahu, D.K., Gagliuffi, D.B., Magain, P., Queloz, D., and Queloz, D. (2016) Temperate Earth-sized planets transiting a nearby ultracool dwarf star. Nature 533:221-224.

Gillon, M., Triaud, A.H.M.J., Demory, B.-O., Jehin, E., Agol, E., Deck, K.M., Lederer, S.M., de Wit, J., Burdanov, A., Ingalls, J.G., Bolmont, E., Leconte, J., Raymond, S.N., Selsis, F., Turbet, M., Barkaoui, K., Burgasser, A., Burleigh, M.R., Carey, S.J., Chaushev, A., Copperwheat, C.M., Delrez, L., Fernandes, C.S., Holdsworth, D.L., Kotze, E.J., Van Grootel, V., Almleaky, Y., Benkhaldoun, Z., Magain, P., and Queloz, D. (2017) Seven temperate terrestrial planets around the nearby ultracool dwarf star TRAPPIST-1. Nature 542:456-460.

Greenblatt, G.D., Orlando, J.J., Burkholder, J.B., and Ravishankara, A.R. (1990) Absorption measurements of oxygen between 330 and $1140 \mathrm{~nm}$. JGR 95:18577.

Greene, T.P., Line, M.R., Montero, C., Fortney, J.J., LustigYaeger, J., and Luther, K. (2016) Characterizing transiting exoplanet atmospheres with JWST. Astrophys $J$ 817:17.

Grenfell, J.L., Gebauer, S., von Paris, P., Godolt, M., and Rauer, H. (2014) Sensitivity of biosignatures on Earth-like planets orbiting in the habitable zone of cool M-dwarf Stars to varying stellar UV radiation and surface biomass emissions. P\&SS 98:66-76.
Grenfell, J.L., Stracke, B., von Paris, P., Patzer, B., Titz, R., Segura, A., and Rauer, H. (2007) The response of atmospheric chemistry on earthlike planets around F, G and K Stars to small variations in orbital distance. P\&SS 55:661671.

Grenfell, J.L., Grießmeier, J.M., von Paris, P., Patzer, A.B.C., Lammer, H., Stracke, B., Gebauer, S., Schreier, F., and Rauer, H. (2012) Response of atmospheric biomarkers to NOx-induced photochemistry generated by stellar cosmic rays for Earth-like planets in the habitable zone of $\mathrm{M}$ dwarf stars. Astrobiology 12:1109-1122.

Grundy, W.M. and Fink, U. (1991) A new spectrum of Triton near the time of the Voyager encounter. Icarus 93:379-385.

Hall, A.E., Schulze, E.D., and Lange, O.L. (1976) Current perspectives of steady-state stomatal responses to environment. In: Water and Plant Life. Springer, Berlin Heidelberg, pp 169-188.

Hamano, K., Abe, Y., and Genda, H. (2013) Emergence of two types of terrestrial planet on solidification of magma ocean. Nature 497:607-610.

Harman, C.E., Schwieterman, E.W., Schottelkotte, J.C., and Kasting, J.F. (2015) Abiotic $\mathrm{O}_{2}$ levels on planets around F, G, $\mathrm{K}$, and M stars: possible false positives for life? ApJ 812:137.

Harman, C.E., Felton, R.C., Domagal-Goldman, S., and Kasting, J.F. (2017) Oxygen false positives in terrestrial planetary atmospheres: an update from the front lines. In Habitable Worlds 2017: A System Science Workshop, November 13-17, 2017, Laramie Wyoming, LPI Contribution No. 2042.

Hazen, R.M., Papineau, D., Bleeker, W., Downs, R.T., Ferry, J.M., McCoy, T.J., Sverjensky, D.A. and Yang, H. (2008) Review Paper. Mineral evolution. Am Mineral 93:1693-1720.

Hegde, S. and Kaltenegger, L. (2013) Colors of extreme exoEarth environments. Astrobiology 13:47-56.

Hegde, S., Paulino-Lima, I.G., Kent, R., Kaltenegger, L., and Rothschild, L. (2015) Surface biosignatures of exo-Earths: remote detection of extraterrestrial life. PNAS 112:38863891.

Heng, K. and Kitzmann, D. (2017) The theory of transmission spectra revisited: a semi-analytical method for interpreting WFC3 data and an unresolved challenge. MNRAS 470:2972.

Hermans, C., Vandaele, A.C., Carleer, M., Fally, S., Colin, R., Jenouvrier, A., Coquart, B., and Mérienne, M.-F. (1999) Absorption cross-sections of atmospheric constituents: NO2, O2, and H2O. Environ Sci Pollut Res 6:151-158.

Hitchcock, D.R. and Lovelock, J.E. (1967) Life detection by atmospheric analysis. Icarus 7:149-159.

Hu, R., Seager, S., and Bains, W. (2012) Photochemistry in terrestrial exoplanet atmospheres. I. Photochemistry model and benchmark cases. ApJ 761:166.

Hu, R., Seager, S., and Bains, W. (2013) Photochemistry in terrestrial exoplanet atmospheres. II. H2S and SO2 photochemistry in anoxic atmospheres. Astrophys J 769:6.

Hunten, D.M. (1973) The escape of light gases from planetary atmospheres. J Atmos Sci 30:1481-1494.

Igamberdiev, A.U. and Lea, P.J. (2006) Land plants equilibrate $\mathrm{O}_{2}$ and $\mathrm{CO}_{2}$ concentrations in the atmosphere. Photosynth Res 87:177-194.

Irwin, P.G.J., Teanby, N.A., De Kok, R., Fletcher, L.N., Howett, C.J.A., Tsang, C.C.C., Wilson, C.F., Calcutt, S.B., Nixon, C.A., and Parrish, P.D. (2008) The NEMESIS planetary atmosphere radiative transfer and retrieval tool. J Quant Spectrosc Radiat Transfer 109:1136-1150.

Izon, G., Zerkle, A.L., Williford, K.H., Farquhar, J., Poulton, S.W., and Claire, M.W. (2017) Biological regulation of at- 
mospheric chemistry en route to planetary oxygenation. Proc Natl Acad Sci U S A 114:E2571-E2579.

Johnson, B. and Goldblatt, C. (2015) The nitrogen budget of Earth. Earth Sci Rev 148:150-173.

Kaltenegger, L., Henning, W.G., and Sasselov, D.D. (2010) Detecting volcanism on extrasolar planets. Astron J 140: 1370.

Kaltenegger, L. and Sasselov, D. (2009) Detecting planetary geochemical cycles on exoplanets: atmospheric signatures and the case of SO2. Astrophys J 708:1162.

Kane, S.R., Kopparapu, R.K., and Domagal-Goldman, S.D. (2014) On the frequency of potential Venus analogs from Kepler data. Astrophys J Lett 794:L5.

Kasper, M.E., Beuzit, J.-L., Verinaud, C., Yaitskova, N., Baudoz, P., Boccaletti, A., Gratton, R.G., Hubin, N., Kerber, F., Roelfsema, R., Schmid, H.M., Thatte, N.A., Dohlen, K., Feldt, M., Venema, L., and Wolf, S. (2008) EPICS: the exoplanet imager for the E-ELT. In Adaptive Optics Systems, edited by N. Hubin, C.E. Max, and P.L. Wizinowich. Proceedings of the SPIE, Volume 7015, Article Id. 70151S, 12 pp. p. 70151S. DOI:10.1117/12.789047

Kasting, J.F. (1997) Habitable zones around low mass stars and the search for extraterrestrial life. Orig Life Evol Biosphere 27:291-3107.

Kasting, J.F. (2001) Earth history: the rise of atmospheric oxygen. Science 293:819-820.

Kasting, J.F. (2013) What caused the rise of atmospheric O2? Chem Geol v362:13-25.

Kasting, J.F. and Canfield, D.E. (2012) The global oxygen cycle. Fundam Geobiol 93-104.

Kasting, J.F. and Donahue, T.M. (1980) The evolution of atmospheric ozone. J Geophys Res 85:3255-3263.

Kasting, J.F., Whitmire, D.P., and Reynolds, R.T. (1993) Habitable zones around main sequence stars. Icarus 101:108-128.

Kasting, J.F., Kopparapu, R., Ramirez, R.M., and Harman, C.E. (2013) Remote life-detection criteria, habitable zone boundaries, and the frequency of Earth-like planets around $\mathrm{M}$ and late K stars. Proc Natl Acad Sci U S A 111:1-6.

Kawahara, H. and Fujii, Y. (2010) Global mapping of Earthlike exoplanets from scattered light curves. Astrophys $J$ 720: 1333.

Keeling, C.D. (1960) The concentration and isotopic abundances of carbon dioxide in the atmosphere. Tellus 12:200 203.

Keeling, C.D., Bacastow, R.B., Bainbridge, A.E., Ekdahl, Jr., C.A., Guenther, P.R., Waterman, L.S., and Chin, J.F.S. (1976) Atmospheric carbon dioxide variations at Mauna Loa Observatory, Hawaii. Tellus A 28:538-551.

Kendall, B., Reinhard, C.T., Lyons, T.W., Kaufman, A.J., Poulton, S.W., and Anbar, A.D. (2010) Pervasive oxygenation along late Archaean ocean margins. Nat Geosci 3:647-652.

Kiang, N.Y., Segura, A., Tinetti, G., Govindjee, Blankenship, R.E., Cohen, M., Siefert, J., Crisp, D., and Meadows, V.S. (2007a). Spectral signatures of photosynthesis. II. Coevolution with other stars and the atmosphere on extrasolar worlds. Astrobiology 7:252-274.

Kiang, N.Y., Siefert, J., Govindjee, Blankenship, R.E. (2007b). Spectral signatures of photosynthesis. I. Review of earth organisms. Astrobiology 7:222-251.

Knoll, A.H., Javaux, E.J., Hewitt, D., and Cohen, P. (2006) Eukaryotic organisms in Proterozoic oceans. Philos Trans $R$ Soc B Biol Sci 361:1023-1038.

Kopparapu, R.K., Ramirez, R., Kasting, J.F., Eymet, V., Robinson, T.D., Mahadevan, S., Terrien, R.C., Domagal-Goldman, S.,
Meadows, V., and Deshpande, R. (2013) Habitable zones around main-sequence stars: new estimates. Astrophys $J$ 765(2):131.

Kopparapu, R.K., Ramirez, R.M., SchottelKotte, J., Kasting, J.F., Domagal-Goldman, S., and Eymet, V. (2014) Habitable zones around main-sequence stars: dependence on planetary mass. Astrophys J Lett 787:L29.

Kreidberg, L. and Loeb, A. (2016) Prospects for characterizing the atmosphere of Proxima Centauri b. Astrophys J Lett 832: L12.

Kreidberg, L., Bean, J.L., Désert, J.M., Benneke, B., Deming, D., Stevenson, K.B., Seager, S., Berta-Thompson, Z., Seifahrt, A., and Homeier, D. (2014) Clouds in the atmosphere of the super-Earth exoplanet GJ 1214b. Nature 505:69-72.

Krissansen-Totton, J., Buick, R., and Catling, D.C. (2015) A statistical analysis of the carbon isotope record from the Archean to Phanerozoic and implications for the rise of oxygen. Am J Sci 315:275-316.

Krissansen-Totton, J., Bergsman, D., and Catling, D.C. (2016a) On detecting biospheres from chemical thermodynamic disequilibrium in planetary atmospheres. Astrobiology 16:39-67.

Krissansen-Totton, J., Schwieterman, E.W., Charnay, B., Arney, G., Robinson, T.D., Meadows, V., and Catling, D.C. (2016b) Is the pale blue dot unique? Optimized photometric bands for identifying earth-like exoplanets. ApJ 817:31.

Kump, L.R. and Barley, M.E. (2007) Increased subaerial volcanism and the rise of atmospheric oxygen 2.5 billion years ago. Nature 448:1033-1036.

Lafferty, W.J., Solodov, A.M., Weber, A., Olson, W.B., and Hartmann, J.M. (1996) Infrared collision-induced absorption by $\mathrm{N}(2)$ near $4.3 \mu \mathrm{m}$ for atmospheric applications: measurements and empirical modeling. Appl Opt 35:5911-5917.

Lederberg, J. (1965) Signs of life: criterion-system of exobiology. Nature 207:9-13.

Lee, C.T., Yeung, L.Y., McKenzie, N.R., Yokoyama, Y., Ozaki, K., and Lenardic, A. (2016) Two-step rise of atmospheric oxygen linked to the growth of continents. Nat Geosci 9:417-424.

Léger, A., Fontecave, M., Labeyrie, A., Samuel, B., Demangeon, O., and Valencia, D. (2011) Is the presence of oxygen on an exoplanet a reliable biosignature? Astrobiology 11:335-341.

Lepland, A., Arrhenius, G., and Cornell, D. (2002) Apatite in early Archean Isua supracrustal rocks, southern West Greenland: its origin, association with graphite and potential as a biomarker. Precambrian Res 118:221-241.

Line, M.R., Knutson, H., Wolf, A.S., and Yung, Y.L. (2014) A systematic retrieval analysis of secondary eclipse spectra. II. A uniform analysis of nine planets and their $\mathrm{C}$ to $\mathrm{O}$ Ratios. Astrophys J 783:70.

Line, M.R. and Parmentier, V. (2016) The influence of nonuniform cloud cover on transit transmission spectra. Astrophys $J$ 820:78.

Lissauer, J.J. (2007) Planets formed in habitable zones of $\mathrm{M}$ dwarf stars probably are deficient in volatiles. ApJ 660:L149L152.

Livengood, T.A., Deming, L.D., A'Hearn, M.F., Charbonneau, D., Hewagama, T., Lisse, C.M., McFadden, L.A., Meadows, V.S., Robinson, T.D., Seager, S., and Wellnitz, D.D. (2011) Properties of an Earth-like planet orbiting a Sun-like star: earth observed by the EPOXI mission. Astrobiology 11:907930.

Lovelock, J.E. (1965) A physical basis for life detection experiments. Nature 207:568-570. 
Lovelock, J.E. and Kaplan, I.R. (1975) Thermodynamics and the recognition of alien biospheres [and Discussion]. Proc $R$ Soc B Biol Sci 189:167-181.

Lovis, C., Snellen, I., Mouillet, D., Pepe, F., Wildi, F., Astudillo-Defru, N., Beuzit, J.L., Bonfils, X., Cheetham, A., Conod, U., and Delfosse, X. (2017) Atmospheric characterization of Proxima $b$ by coupling the SPHERE high-contrast imager to the ESPRESSO spectrograph. A\&A 599:A16.

Luger, R. and Barnes, R. (2015) Extreme water loss and abiotic O2 buildup on planets throughout the habitable zones of $\mathrm{M}$ dwarfs. Astrobiology 15:119-143.

Luger, R., Lustig-Yaeger, J., Fleming, D.P., Tilley, M.A., Agol, E., Meadows, V.S., Deitrick, R., and Barnes, R. (2017) The pale green dot: a method to characterize Proxima Centauri b using Exo-Aurorae. Astrophys $J$ 837:63.

Luo, G., Ono, S., Beukes, N.J., Wang, D.T., Xie, S., and Summons, R.E. (2016) Rapid oxygenation of Earths atmosphere 2.33 billion years ago. Science Adv 2:e1600134.

Lustig-Yaeger, J., Tovar, G., Schwieterman, E.W., Fujii, Y., and Meadows, V.S., Meadows (2017) Detecting oceans on exoplanets using phase-dependent mapping with next generation coronagraph-equipped telescopes, Habitable Worlds 2017, LPI Contribution No. 2042. Available online at www.hou.usra.edu/meetings/habitableworlds2017/pdf/4110 .pdf

Lyons, T.W., Reinhard, C.T., and Planavsky, N.J. (2014) The rise of oxygen in Earth's early ocean and atmosphere. Nature 506:307-315.

Madhusudhan, N. and Seager, S. (2009) A temperature and abundance retrieval method for exoplanet atmospheres. Astrophys $J$ 707:24.

Maté, B., Lugez, C., Fraser, G.T., and Lafferty, W.J. (1999) Absolute intensities for the $\mathrm{O} 21.27$ continuum absorption. $J$ Geophys Res 104:585-590.

Maté, B., Lugez, C.L., Solodov, A.M., Fraser, G.T., and Lafferty, W.J. (2000) Investigation of the collision-induced absorption by $\mathrm{O}_{2}$ near $6.4 \mu \mathrm{m}$ in pure $\mathrm{O}_{2}$ and $\mathrm{O}_{2} / \mathrm{N}_{2}$ mixtures. $J$ Geophys Res 105:222-225, 230.

McCollom, T.M. and Seewald, J.S. (2006) Carbon isotope composition of organic compounds produced by abiotic synthesis under hydrothermal conditions. Earth Planet Sci Lett 243:74-84.

Meadows, V.S. (2008) Planetary environmental signatures of habitability and life. In: Exoplanets: Detection, Formation, Properties, Habitability, edited by J. Mason. Springer Praxis Books. ISBN 978-3-540-74007-0. Chichester, UK: Praxis Publishing Ltd, p 259.

Meadows, V.S. (2017) Reflections on $\mathrm{O}_{2}$ as a biosignature in exoplanetary atmospheres. Astrobiology 17:1022-1052.

Meadows, V.S., Arney, G.N., Schwieterman, E.W., LustigYaeger, J., Lincowski, A.P., Robinson, T., DomagalGoldman, S.D., Barnes, R.K., Fleming, D.P., Deitrick, R., Luger, R., Driscoll, P.E., Quinn, T.R., and Crisp, D. (2018) The habitability of Proxima Centauri b: environmental states and observational discriminants. Astrobiology 18:133-189.

Mennesson, B., Gaudi, S., Seager, S., Cahoy, K., DomagalGoldman, S., Feinberg, L., Guyon, O., Kasdin, J., Marois, C., Mawet, D., Tamura, M., Mouillet, D., Prusti, T., Quirrenbach, A., Robinson, T., Rogers, L., Scowen, P., Somerville, R., Stapelfeldt, K., Stern, D., Still, M., Turnbull, M., Booth, J., Kiessling, A., Kuan, G., and Warfield, K. (2016) The Habitable Exoplanet (HabEx) Imaging Mission: preliminary science drivers and technical requirements. In Proc. SPIE 9904, Space Telescopes and Instrumentation 2016: Optical, Infra- red, and Millimeter Wave, edited by H.A. MacEwen, G.G. Fazio, M. Lystrup, N. Batalha, N. Siegler, and E.C. Tong. International Society for Optics and Photonics, p 99040L. DOI: $10.1117 / 12.2240457$

Misra, A.K. and Meadows, V.S. (2014). Discriminating between cloudy, hazy, and clear sky exoplanets using refraction. Astrophys J Lett 795:L14.

Misra, A., Meadows, V., Claire, M., and Crisp, D. (2014a). Using dimers to measure biosignatures and atmospheric pressure for terrestrial exoplanets. Astrobiology 14:67-86.

Misra, A., Meadows, V., and Crisp, D. (2014b). The Effects of refraction on transit transmission spectroscopy: application to Earth-like exoplanets. ApJ 792:61-72.

Misra, A., Krissansen-Totton, J., Koehler, M.C., and Sholes, S. (2015) Transient sulfate aerosols as a signature of exoplanet volcanism. Astrobiology 15:462-477.

Mojzsis, S.J., Arrhenius, G., McKeegan, K.D., Harrison, T.M., Nutman, A.P., and Friend, C.R.L. (1996) Evidence for life on Earth before 3,800 million years ago. Nature 384:55.

Morley, C.V., Fortney, J.J., Marley, M.S., Zahnle, K., Line, M., Kempton, E., Lewis, N., and Cahoy, K. (2015) Thermal emission and reflected light spectra of super Earths with flat transmission spectra. Astrophys $J$ 815:110.

Murata, N., Takahashi, S., Nishiyama, Y., and Allakhverdiev, S.I. (2007) Photoinhibition of photosystem II under environmental stress. Biochim Biophys Acta 1767:414-421.

Narita, N., Enomoto, T., Masaoka, S., and Kusakabe, N. (2015) Titania may produce abiotic oxygen atmospheres on habitable exoplanets. Sci Rep 5:13977.

Nutman, A.P., Bennett, V.C., Friend, C.R., Van Kranendonk, M.J., and Chivas, A.R. (2016) Rapid emergence of life shown by discovery of 3,700-million-year-old microbial structures. Nature 537:535-538.

Olson, J.M. (1970) The evolution of photosynthesis. Science (New York, NY) 168:438.

Olson, J.M. (1978) Precambrian evolution of photosynthetic and respiratory organisms. In Evolutionary Biology, Springer, Boston, pp 1-37.

Olson, J.M. (1980) Chlorophyll organization in green photosynthetic bacteria. Biochim Biophys Acta 594:33-51.

Olson, J.M. (2006) Photosynthesis in the Archean era. Photosynth Res 88:109-117.

Olson, J.M. and Pierson, B.K. (1986) Origin and evolution of photosynthetic reaction centers. Orig Life Evol Biosph 16: 361-362.

Olson, S.L., Reinhard, C.T., and Lyons, T.W. (2016) Limited role for methane in the mid-Proterozoic greenhouse. PNAS 113:11447-11452.

Pallé, E., Osorio, M.R.Z., Barrena, R., Montañés-Rodríguez, P., and Martín, E.L. (2009) Earth's transmission spectrum from lunar eclipse observations. Nature 459:814-816.

Pavlov, A.A., Hurtgen, M.T., Kasting, J.F., and Arthur, M.A. (2003) Methane-rich Proterozoic atmosphere?. Geology 31:87-90.

Pavlov, A.A. and Kasting, J.F. (2002) Mass-independent fractionation of sulfur isotopes in Archean sediments: strong evidence for an anoxic Archean atmosphere. Astrobiology 2: 27-41.

Pierson, B.K. and Olson, J.M. (1989) Evolution of photosynthesis in anoxygenic photosynthetic procaryotes. In Microbial Mats, Physiological Ecology of Benthic Communities, edited by Y. Cohen and E. Rosenberg, American Society of Microbiology, Washington, DC, pp 402-427.

Pierson, B.K., Mitchell, H.K., and Ruff-Roberts, A.L. (1993) Chloroflexus aurantiacus and ultraviolet radiation: implica- 
tions for archean shallow-water stromatolites. Orig Life Evol Biosph 23:243-260.

Pilcher, C.B. (2003) Biosignatures of early earths. Astrobiology 3:471-486.

Planavsky, N.J., Asael, D., Hofmann, A., Reinhard, C.T., Lalonde, S.V, Knudsen, A., Wang, X., Ossa Ossa, F., Pecoits, E., Smith, A.J.B., Beukes, N.J., Bekker, A., Johnson, T.M., Konhauser, K.O., Lyons, T.W., and Rouxel, O.J. (2014a). Evidence for oxygenic photosynthesis half a billion years before the Great Oxidation Event. Nat Geosci 7:283-286.

Planavsky, N.J., Reinhard, C.T., Wang, X., Thomson, D., Mcgoldrick, P., Rainbird, R.H., Johnson, T., Fischer, W.W., and Lyons, T.W. (2014b). Low mid-proterozoic atmospheric oxygen levels and the delayed rise of animals. Science 346:635-638.

Postman, M., Brown, T., Sembach, K., Giavalisco, M., Traub, W., Stapelfeldt, K., Calzetti, D., Oegerle, W., Rich, R.M., Stahl, H.P., Tumlinson, J., Mountain, M., Soummer, R., and Hyde, T. (2010) Science drivers and requirements for an Advanced Technology Large Aperture Space Telescope (ATLAST): implications for technology development and synergies with other future facilities. SPIE 7731:12.

Quanz, S.P., Crossfield, I., Meyer, M.R., Schmalzl, E., and Held, J. (2015) Direct detection of exoplanets in the 3-10 $\mu \mathrm{m}$ range with E-ELT/METIS. Int J Astrobiol 14:279-289.

Ratner, M.I. and Walker, J.C.G. (1972) Atmospheric ozone and the history of life. J Atmos Sci 29:803-808.

Rauer, H., Catala, C., Aerts, C., Appourchaux, T., Benz, W., Brandeker, A., Christensen-Dalsgaard, J., Deleuil, M., Gizon, L., Goupil, M.J., and Güdel, M. (2014) The PLATO 2.0 mission. Exp Astron 38:249-330.

Rauer, H., Gebauer, S.V., Paris, P.V., Cabrera, J., Godolt, M., Grenfell, J.L., Belu, A., Selsis, F., Hedelt, P., and Schreier, F. (2011) Potential biosignatures in super-Earth atmospheres-I. Spectral appearance of super-Earths around $\mathrm{M}$ dwarfs. $A \& A$ 529:A8.

Rauscher, B.J., Bolcar, M.R., Clampin, M., Domagal-Goldman, S.D., McElwain, M.W., Moseley, S.H., Stahle, C., Stark, C.C., and Thronson, H.A. (2015) ATLAST detector needs for direct spectroscopic biosignature characterization in the visible and near-IR. SPIE 9602-9612. ArXiv: 1508.06661.

Raymond, S.N., Scalo, J., and Meadows, V.S. (2007) A decreased probability of habitable planet formation around lowmass stars. Astrophys J 669:606; arXiv:0707.1711v1.

Reinhard, C.T., Olson, S.L., Schwieterman, E.W., and Lyons, T.W. (2017) False negatives for remote life detection on ocean-bearing planets: lessons from the early Earth. Astrobiology 17:287-297.

Reinhard, C.T., Planavsky, N.J., Olson, S.L., Lyons, T.W., and Erwin, D.H. (2016) Earth's oxygen cycle and the evolution of animal life. PNAS 113:8933-8938.

Ribas, I., Bolmont, E., Selsis, F., Reiners, A., Leconte, J., Raymond, S.N., Engle, S.G., Guinan, E.F., Morin, J., Turbet, M., Forget, F., and Anglada-Escude, G. (2016) The habitability of Proxima Centauri b. I. Irradiation, rotation and volatile inventory from formation to the present. A\&A 596: A111.

Richard, C., Gordon, I.E., Rothman, L.S., Abel, M., Frommhold, L., Gustafsson, M., Hartmann, J.M., Hermans, C., Lafferty, W.J., Orton, G.S., Smith, K.M., and Tran, H. (2012) New section of the HITRAN database: collision-induced absorption (CIA). JQSRT 113:1276-1285.

Ricker, G.R., Winn, J.N., Vanderspek, R., Latham, D.W., Bakos, G.Á., Bean, J.L., Berta-Thompson, Z.K., Brown, T.M., Buchhave, L., Butler, N.R. and Butler, R.P. (2015) Transiting exoplanet survey satellite. J Astron Telesc Instrum Syst 1, 014003.

Riding, R., Fralick, P., and Liang, L. (2014) Identification of an Archean marine oxygen oasis. Precambrian Res 251:232-237.

Roberson, A.L., Roadt, J., Halevy, I., and Kasting, J.F. (2011) Greenhouse warming by nitrous oxide and methane in the Proterozoic Eon. Geobiology 9:313-320.

Robinson, T.D., Ennico, K., Meadows, V.S., Sparks, W., Bussey, D.B.J., Schwieterman, E.W., and Breiner, J. (2014) Detection of ocean glint and ozone absorption using LCROSS Earth observations. Astrophys $J$ 787:171.

Robinson, T.D., Meadows, V.S., and Crisp, D. (2010) Detecting oceans on extrasolar planets using the glint effect. ApJ 721: L67-L71.

Robinson, T.D., Stapelfeldt, K.R., and Marley, M.S. (2016) Characterizing rocky and gaseous exoplanets with $2 \mathrm{~m}$ class space-based coronagraphs. Publ Astron Soc Pac 128:025003.

Rodler, F. and López-Morales, M. (2014) Feasibility studies for the detection of $\mathrm{O} 2$ in an Earth-like exoplanet. Astrophys $J$ 781:54.

Rogers, L.A. (2015) Most 1.6 Earth-radius planets are not rocky. Astrophys $J$ 801:41.

Rosenqvist, J. and Chassefière, E. (1995) Inorganic chemistry of $\mathrm{O}_{2}$ in a dense primitive atmosphere. $P \& S S$ 43:3-10.

Rosing, MT. (1999) ${ }^{13}$ C-depleted carbon microparticles in $>3700$-Ma sea-floor sedimentary rocks from West Greenland. Science 283:674-676.

Rosing, M.T. and Frei, R. (2004) U-rich Archaean sea-floor sediments from Greenland-indications of $>3700$ Ma oxygenic photosynthesis. Earth Planet Sci Lett 217:237244.

Rothman, L.S., Gordon, I.E., Babikov, Y., Barbe, A., Chris Benner, D., Bernath, P.F., Birk, M., Bizzocchi, L., Boudon, V., Brown, L.R., Campargue, A., Chance, K., Cohen, E.A., Coudert, L.H., Devi, V.M., Drouin, B.J., Fayt, A., Flaud, J.M., Gamache, R.R., Harrison, J.J., Hartmann, J.-M., Hill, C., Hodges, J.T., Jacquemart, D., Jolly, A., Lamouroux, J., Le Roy, R.J., Li, G., Long, D.A., Lyulin, O.M., Mackie, C.J., Massie, S.T., Mikhailenko, S., Müller, H.S.P., Naumenko, O.V., Nikitin, A.V., Orphal, J., Perevalov, V., Perrin, A., Polovtseva, E.R., Richard, C., Smith, M.A.H., Starikova, E., Sung, K., Tashkun, S., Tennyson, J., Toon, G.C., Tyuterev, V.G., and Wagner, G. (2013) The HITRAN2012 molecular spectroscopic database. JQSRT 130: 4-50.

Rugheimer, S., Kaltenegger, L., Zsom, A., Segura, A., and Sasselov, D. (2013) Spectral fingerprints of Earth-like planets around FGK stars. Astrobiology 13:251-269.

Rugheimer, S., Kaltenegger, L., Segura, A., Linsky, J., and Mohanty, S. (2015) Effect of UV radiation on the spectral fingerprints of earth-like planets orbiting M stars. ApJ 809:57.

Schaefer, L., Wordsworth, R., Berta-Thompson, Z., Sasselov, D., Blamey, N.J.F., Brand, U., Parnell, J., Spear, N., Lécuyer, C., and Benison, K. (2016) Predictions of the atmospheric composition of GJ 1132b 651-654. DOI:10.1130/ G37937.1

Schindler, T.L. and Kasting, J.F. (2000) Synthetic spectra of simulated terrestrial atmospheres containing possible biomarker gases. Icarus 145:262-271.

Schwieterman, E.W., Cockell, C.S., and Meadows, V.S. (2015a). Nonphotosynthetic pigments as potential biosignatures. Astrobiology 15:341-361.

Schwieterman, E.W., Robinson, T.D., Meadows, V.S., Misra, A., and Domagal-Goldman, S. (2015b). Detecting and constraining 
$\mathrm{N} 2$ abundances in planetary atmospheres using collisional pairs. ApJ 810:57.

Schwieterman, E.W., Meadows, V.S., Domagal-Goldman, S.D., Deming, D., Arney, G.N., Luger, R., Harman, C.E., Misra, A., and Barnes, R. (2016) Identifying planetary biosignature impostors: spectral features of $\mathrm{CO}$ And $\mathrm{O} 4$ resulting from abiotic $\mathrm{O}_{2} / \mathrm{O}_{3}$ production. ApJ 819:L13.

Schwieterman, E.W., Kiang, N.Y., Parenteau, M.N., Harman, C.E., DasSarma, S., Fisher, T.M., Arney, G.N., Hartnett, H.E., Reinhard, C.T., Olson, S.L., Meadows, V.S., Cockell, C.S., Walker, S.I., Grenfell, J.L., Hegde, S., Rugheimer, S., Hu, R., and Lyons, T.W. (2018). Exoplanet biosignatures: a review of remotely detectable signs of life. Astrobiology 18:663-708.

Seager, S. and Bains, W. (2015) The search for signs of life on exoplanets at the interface of chemistry and planetary science. Sci Adv 1:e1500047.

Seager, S., Schrenk, M., and Bains, W. (2012) An astrophysical view of Earth-based metabolic biosignature gases. Astrobiology 12:61-82.

Seager, S., Cash, W., Domagal-Goldman, S., Kasdin, N.J., Kuchner, M., Roberge, A., Shaklan, S., Sparks, W., Thomson, M., Turnbull, M., and Warfield, K. (2015) Exo-S: starshade probe-class exoplanet direct imaging mission concept Final Report. Available online at exep.jpl.nasa.gov/stdt

Segura, A., Krelove, K., Kasting, J.F., Sommerlatt, D., Meadows, V., Crisp, D., Cohen, M., and Mlawer, E. (2003) Ozone concentrations and ultraviolet fluxes on Earth-like planets around other stars. Astrobiology 3:689-708.

Segura, A., Kasting, J.F., Meadows, V., Cohen, M., Scalo, J., Crisp, D., Butler, R.A.H., and Tinetti, G. (2005) Biosignatures from Earth-like planets around $\mathrm{M}$ dwarfs. Astrobiology 5:706-725.

Segura, A., Walkowicz, L.M., Meadows, V., Kasting, J., and Hawley, S. (2010) The effect of a strong stellar flare on the atmospheric chemistry of an earth-like planet orbiting an M dwarf. Astrobiology 10:751-771.

Selsis, F., Despois, D., and Parisot, J.P. (2002) Signature of life on exoplanets: can Darwin produce false positive detections? A\&A 388:985-1003.

Senge, M.O. and Smith, K.M. (1995) Biosynthesis and structure of the bacteriochlorophylls. In Anoxygenic Photosynthetic Bacteria, edited by R.E. Blankenship, M.T. Madigan, and C.E. Bauer. Springer, Netherlands, pp 137-151.

Shapiro, M.M. and Gush, H.P. (1966) The collision-induced fundamental and first overtone bands of oxygen and nitrogen. Can J Phys 44:949-963.

Sheldon, N.D. (2006) Precambrian paleosols and atmospheric $\mathrm{CO}_{2}$ levels. Precambrian Res 147:148-155.

Shen, Y., Buick, R., and Canfield, D.E. (2001) Isotopic evidence for microbial sulphate reduction in the early Archaean era. Nature 410:77-81.

Sleep, N.H. (2010) The Hadean-Archaean environment. Cold Spring Harb Perspect Biol 2:a002527.

Snellen, I.A.G., de Kok, R., Birkby, J.L., Brandl, B., Brogi, M., Keller, C.U., Kenworthy, M., Schwarz, H., and Stuik, R. (2015) Combining high-dispersion spectroscopy with high contrast imaging: probing rocky planets around our nearest neighbors. $A \& A$ 576:A59.

Snellen, I.A.G., de Kok, R.J., Le Poole, R., Brogi, M., and Birkby, J. (2013) Finding extraterrestrial life using groundbased high-dispersion spectroscopy. Astrophys J 764:182.

Soo, R.M., Hemp, J., Parks, D.H., Fischer, W.W., and Hugenholtz, P. (2017) On the origins of oxygenic photosynthesis and aerobic respiration in Cyanobacteria. Science 355:1436-1440.
Sozzetti, A. (2014) Exoplanets with Gaia: synergies in the Making. Eur Astron Soc Publ Ser 68:93-99.

Stapelfeldt, K.R., Belikov, R., Bryden, G., Cahoy, K., Chakrabarti, S., Marley, M. McElwain, M., Meadows, V., Serabyn, E., Trauger, J., Dekens, F., Brenner, M., Brugarolas, P. Dubovitsky, S., Effinger, R., Heeg, C., Hirsch, B., Kissel, A., Krist, J., Lange, J., Nissen, J., Oseas, J., Pong, C., Rubio, M., Sunada, E., Warfield, K., Domagal-Goldman, S., Kuchner, M., Lawson, P., Morley, C., Menati, B., Roberge, A., Robinson, T., Sandhu, J., Seager, S., and Unwin, S. (2015) Exo-C Mission Study Final Report, NASA Exoplanet Exploration Program internal document, available at https://exoplanets.nasa.gov/exep/stdt/ Exo-C_Final_Report_for_Unlimited_Release_150323.pdf

Stephan, K., Jaumann, R., Brown, R.H., Soderblom, J.M., Soderblom, L.A., Barnes, J.W., Sotin, C., Griffith, C.A., Kirk, R.L., Baines, K.H., and Buratti, B.J. (2010) Specular reflection on Titan: liquids in Kraken Mare. Geophys Res Lett 37: L07104.

Stock, J.W., Blaszczak-Boxe, C.S., Lehmann, R., Grenfell, J.L., Patzer, A.B.C., Rauer, H., and Yung, Y.L. (2017) A detailed pathway analysis of the chemical reaction system generating the Martian vertical ozone profile. Icarus 291: 192-202.

Sullivan, P.W., Winn, J.N., Berta-Thompson, Z.K., Charbonneau, D., Deming, D., Dressing, C.D., Latham, D.W., Levine, A.M., McCullough, P.R., Morton, T., and Ricker, G.R. (2015) The Transiting Exoplanet Survey Satellite: simulations of planet detections and astrophysical false positives. Astrophys J 809:77.

Szentgyorgyi, A., Baldwin, D., Barnes, S., Bean, J., Ben-Ami, S., Brennan, P., Budynkiewicz, J., Chun, M.Y., Conroy, C., Crane, J.D., Epps, H., Evans, I., Evans, J., Foster, J., Frebel, A., Gauron, T., Guzmán, D., Hare, T., Jang, B.H., Jang, J.G., Jordan, A., Kim, J., Kim, K.M., de Oliveira, C.M., LopezMorales, M., McCracken, K., McMuldroch, S., Miller, J., Mueller, M., Oh, J.S., Onyuksel, C., Ordway, M., Park, B.G., Park, C., Park, S.J., Paxson, C., Phillips, D., Plummer, D., Podgorski, W., Seifahrt, A., Stark, D., Steiner, J., Uomoto, A., Walsworth, R., and Yu, Y.S. (2016) The GMT-Consortium Large Earth Finder (G-CLEF): an optical Echelle spectrograph for the Giant Magellan Telescope (GMT). In GroundBased and Airborne Instrumentation for Astronomy VI. Proc. SPIE 990822.

Tang, D., Shi, X., Wang, X., and Jiang, G. (2016) Extremely low oxygen concentration in mid-Proterozoic shallow seawaters. Precambrian Res 276:145-157.

Tian, F. (2015) History of water loss and atmospheric $\mathrm{O}_{2}$ buildup on rocky exoplanets near M dwarfs. Earth Planet Sci Lett 432:126-132.

Tian, F., France, K., Linsky, J.L., Mauas, P.J.D., and Vieytes, M.C. (2014) High stellar FUV/NUV ratio and oxygen contents in the atmospheres of potentially habitable planets. Earth Planet Sci Lett 385:22-27.

Tinetti, G., Meadows, V.S., Crisp, D., Kiang, N.Y., Kahn, B.H., Bosc, E., Fishbein, E., Velusamy, T., and Turnbull, M. (2006) Detectability of planetary characteristics in disk-averaged spectra II: synthetic spectra and light-curves of earth. Astrobiology 6:881-900.

Thalman, R. and Volkamer, R. (2013) Temperature dependent absorption cross-sections of $\mathrm{O}_{2}-\mathrm{O}_{2}$ collision pairs between 340 and $630 \mathrm{~nm}$ and at atmospherically relevant pressure. Phys chem chem phys 15:15371-15381.

Trouwborst, R.E., Johnston, A., Koch, G., Luther, III G.W., and Pierson, B.K. (2007) Fe(II) oxidation mediated by photo- 
synthetic activity of Yellowstone hot spring microbial mats: model for Precambrian Fe(II) oxidation. Geochim Cosmochim Acta 71:4629-4643.

Turbet, M., Leconte, J., Selsis, F., Bolmont, E., Forget, F., Ribas, I., Raymond, S.N., and Anglada-Escudé, G. (2016) The habitability of Proxima Centauri b-II. Possible climates and observability. A\&A 596:A112.

Ueno, Y., Yamada, K., Yoshida, N., Maruyama, S., and Isozaki, Y. (2006) Evidence from fluid inclusions for microbial methanogenesis in the early Archaean era. Nature 440:516-519.

Ueno, Y., Johnson, M.S., Danielache, S.O., Eskebjerg, C., Pandey, A., and Yoshida, N. (2009) Geological sulfur isotopes indicate elevated OCS in the Archean atmosphere, solving faint young sun paradox. Proc Natl Acad Sci U S A 106:14784-14789.

Unterborn, C.T. and Panero, W.R. (2017) The effects of $\mathrm{Mg} / \mathrm{Si}$ on the exoplanetary refractory oxygen budget. Astrophys $J$ 845:61.

Van Zuilen, M.A., Lepland, A., and Arrhenius, G. (2002) Reassessing the evidence for the earliest traces of life. Nature 418:627-630.

von Paris, P., Grenfell, J.L., Rauer, H., and Stock, J.W. (2013a). $\mathrm{N}_{2}$-associated surface warming on early Mars. Planet Space Sci 82:149-154.

von Paris, P., Hedelt, P., Selsis, F., Schreier, F., and Trautmann, T. (2013b). Characterization of potentially habitable planets: retrieval of atmospheric and planetary properties from emission spectra. A\&A 551:A120.

Waldmann, I.P., Tinetti, G., Rocchetto, M., Barton, E.J., Yurchenko, S.N., and Tennyson, J. (2015) Tau-REx I: a next generation retrieval code for exoplanetary atmospheres. Astrophys $J$ 802:107.

Walker, J.C.G. (1977) Evolution of the Atmosphere. Macmillan, New York.

Walker, J.C.G., Hays, P.B., and Kasting, J.F. (1981) A negative feedback mechanism for the long-term stabilization of Earth's surface temperature. J Geophys Res 86:9776-9782.

Walker, S.I., Bains, W., Cronin, L., DasSarma, S., Danielache, S., Domagal-Goldman, S., Kacar, B., Kiang, N.Y., Lenardic, A., Reinhard, C.T., Moore, W., Schwieterman, E.W., Shkolnik, E.L., and Smith, H.B. (2018) Exoplanet biosignatures: future directions. Astrobiology 18:779-824.

Weiss, L.M. and Marcy, G.W. (2014) The mass-radius relation for 65 exoplanets smaller than 4 Earth radii. Astrophys J Lett 783:L6.

Westall, F., Cavalazzi, B., Lemelle, L., Marrocchi, Y., Rouzaud, J.N., Simionovici, A., Salomé, M., Mostefaoui, S., Andreazza, C., Foucher, F., and Toporski, J. (2011) Implications of in situ calcification for photosynthesis in a $\sim 3.3$
Ga-old microbial biofilm from the Barberton greenstone belt, South Africa. Earth Planet Sci Lett 310:468-479.

Winn, J.N. (2010) Exoplanet transits and occultations. Exoplanets 1:55-77.

Wordsworth, R.D. (2016) Atmospheric nitrogen evolution on Earth and Venus. Earth Planet Sci Lett 447:103-111.

Wordsworth, R. and Pierrehumbert, R. (2014) Abiotic oxygendominated atmospheres on terrestrial habitable zone planets. ApJ 785:L20.

Young, P.A., Desch, S.J., Anbar, A.D., Barnes, R., Hinkel, N.R., Kopparapu, R., Madhusudhan, N., Monga, N., Pagano, M.D., Riner, M.A., and Scannapieco, E. (2014) Astrobiological stoichiometry. Astrobiology 14:603-626.

Yung, Y.L. and DeMore, W.B. (1999) Photochemistry of Planetary Atmospheres. New York: Oxford University Press.

Zahnle, K., Claire, M., and Catling, D. (2006) The loss of massindependent fractionation in sulfur due to a Palaeoproterozoic collapse of atmospheric methane. Geobiology 4:271-283.

Address correspondence to: Victoria S. Meadows Department of Astronomy University of Washington PO Box 351580 Seattle, WA 98195

E-mail:vsm@astro.washington.edu

Submitted 12 December 2017

Accepted 15 December 2017

Abbreviations Used
ELTs $=$ extremely large telescopes
HabEx $=$ Habitable Exoplanet Imaging Mission
IWA $=$ inner-working angles
JWST $=$ James Webb Space Telescope
LUVOIR $=$ Large UltraViolet Optical Infrared Surveyor
MIR $=$ mid-infrared
NIR $=$ near-infrared
OWAs $=$ outer-working angles
PAL $=$ present atmospheric level
PSI $=$ photosystem I
PSII $=$ photosystem II
RV $=$ radial velocity
S/N $=$ signal-to-noise
WFIRST $=$ Wide Field Infrared Survey Telescope

